\title{
Phase Behavior of Liquid Crystals in Confinement
}

\author{
Dissertation zur Erlangung des \\ mathematisch-naturwissenschaftlichen Doktorgrades \\ „Doctor rerum naturalium “ \\ der Georg-August-Universität Göttingen
}

\author{
vorgelegt von \\ Jonathan Michael Fish \\ aus London, England
}

Göttingen 2011 
D 7

Referent: Prof. Dr. Marcus Müller

Korreferent: Prof. Dr. Reiner Kree

Tag der mündlichen Prüfung: 


\section{Acknowledgments}

Most importantly I would like to thank my supervisor Richard Vink, who has been both a great teacher and has put up with my stupidity for the last three years. I also thank Timo Fischer, who has endured similarly.

Friends, colleagues, and family are too numerous to name individually, but so many have helped me throughout my PhD. I hope that these people know who they are, and that my thanks are without question. 



\section{Contents}

$\begin{array}{lll}1 & \text { An introduction to liquid crystals } & 1\end{array}$

1.1 Motivation $\ldots \ldots \ldots \ldots \ldots \ldots \ldots \ldots \ldots$

1.2 Liquid crystal basics $\ldots \ldots \ldots \ldots \ldots \ldots \ldots$

1.2 .1 Isotropic and nematic phases $\ldots \ldots \ldots \ldots . \ldots 2$

1.2 .2 Smectic phases $\ldots \ldots \ldots \ldots \ldots$. . . . . . . . . 4

$1.2 .3 \quad$ Inducing phase transitions: thermotropic versus lyotropic systems

1.3 Liquid crystal molecules . . . . . . . . . . . . . . . . . . 4

1.3 .1 Small organic molecules $\ldots \ldots \ldots \ldots \ldots$

1.3 .2 Long helical rods $\ldots \ldots \ldots \ldots$

1.3 .3 Amphiphilic compounds . . . . . . . . . . . . . . . . 7

1.3.4 Elastomers. . . . . . . . . . . . . . . . . . . . . . . 7

1.4 Isotropic-nematic transition: bulk theoretical approaches $\ldots \ldots . .99$

1.4 .1 Nematic order parameter . . . . . . . . . . . . . . . . 9 9

1.4 .2 Landau-de Gennes phenomenological theory . . . . . . . . . . 13

$1.4 .3 \quad$ Maier-Saupe theory . . . . . . . . . . . . . . . . . . . . 14

1.4 .4 Onsager theory $\ldots \ldots \ldots \ldots \ldots \ldots \ldots$

1.5 Role of confinement: scope of the thesis . . . . . . . . . . . 18

1.5 .1 Thesis outline $\ldots \ldots \ldots \ldots \ldots$

2 Simulation methods 21

2.1 Introduction . . . . . . . . . . . . . . . . . . . . . . 21

2.2 The Metropolis algorithm . . . . . . . . . . . . . . . . 22

2.2 .1 Thermal equilibrium: Boltzmann distribution . . . . . . . 22

2.2 .2 Importance sampling $\ldots \ldots \ldots \ldots \ldots \ldots \ldots$

2.2.3 Markov chain Monte Carlo: detailed balance and ergodicity . 23

$2.2 .4 \quad$ Metropolis algorithm $\ldots \ldots \ldots \ldots \ldots \ldots$

2.3 Exponential slowing down $\ldots \ldots \ldots \ldots \ldots$

2.4 Biased sampling algorithms $\ldots \ldots \ldots \ldots \ldots \ldots$

2.4 .1 Wang-Landau algorithm . . . . . . . . . . . . . . 27

$2.4 .2 \quad$ Transmission matrix Wang-Landau algorithm . . . . . . . . . 29

$2.4 .3 \quad$ Successive umbrella sampling $\ldots \ldots \ldots \ldots \ldots$. . . . . . . . 32

3 The Lebwohl-Lasher model 35

3.1 Model definition . . . . . . . . . . . . . . . . . . . . . 35 
3.2 The generalized spin flip . . . . . . . . . . . . . . . . . . 36

$3.3 \quad$ Summary of bulk results $\ldots \ldots \ldots$. . . . . . . . . . . . . . . . 38

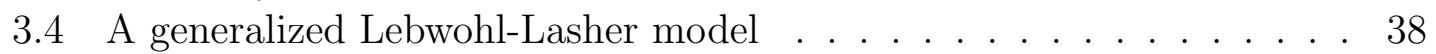

\begin{tabular}{|lll}
\hline 4 & Finite-size scaling of the isotropic-nematic transition & 41
\end{tabular}

4.1 Introduction . . . . . . . . . . . . . . . . . . 41

4.2 Finite-size scaling . . . . . . . . . . . . . . . . . . . . . . . . . . . . . . . . 42

$4.3 \quad$ Simulations using Lebwohl-Lasher type models . . . . . . . . . . . . . 43

4.3 .1 Model and simulation method . . . . . . . . . . . . . . . 44

4.4 Results and analysis . . . . . . . . . . . . . . . . 46

$4.4 .1 \quad$ Determining the order of the transition . . . . . . . . . . . . 46

$4.4 .2 \quad$ Extrapolation of $\epsilon_{L, c}$ : Potts $1 / L^{d}$ scaling . . . . . . . . . . . . 49

4.4.3 Extrapolation of $\epsilon_{L, k}$ : application of the Borgs-Kotecký method 49

4.4 .4 Latent heat density $\ldots \ldots \ldots \ldots 5$

4.5 Summary . . . . . . . . . . . . . . . . . . . . 57

5 Confinement of nematic liquid crystals $\quad \mathbf{5 9}$

5.1 Introduction . . . . . . . . . . . . . . . . . . . . . . 59

5.2 Simulation setup . . . . . . . . . . . . . . . . . . 61

5.3 The two-dimensional limit . . . . . . . . . . . . . . . . . . . . . . . . . 62

5.3 .1 No phase transition . . . . . . . . . . . . . . . . . 64

5.3 .2 A continuous phase transition . . . . . . . . . . . . . 65

$5.3 .3 \quad$ A first-order phase transition . . . . . . . . . . . . . . . . 65

5.3 .4 Summary of results for the 2D case . . . . . . . . . . . . . . 68

5.4 Thin films: between 2D and 3D . . . . . . . . . . . . . . 68

$5.4 .1 \quad$ Finite-film thickness: the case $p=8$. . . . . . . . . . . . . 68

$5.4 .2 \quad$ Finite-film thickness: the case $p=20$. . . . . . . . . . . . . 72

5.5 Application of the Kelvin equation . . . . . . . . . . . . . . . 74

5.6 Summary of simulations between flat walls . . . . . . . . . . . . . 78

\begin{tabular}{|lll}
\hline 6 & Random quenched disorder in liquid crystals & $\mathbf{7 9}$
\end{tabular}

6.1 Introduction . . . . . . . . . . . . . . . . . . . . . 79

$6.1 .1 \quad$ Sprinkled Silica Spin model . . . . . . . . . . . . . . . . . . . 82

6.1 .2 Initial results $\ldots \ldots \ldots \ldots$. . . . . . . . . . . . . 83

6.1 .3 Violation of self-averaging . . . . . . . . . . . . 85

6.2 Fate of the isotropic-nematic transition: the case $p=2 \ldots$. . . . . 86

6.2 .1 The nematic correlation function . . . . . . . . . . . 86

6.2 .2 Pseudo transition temperatures . . . . . . . . . . . . . . . . . 89

6.2 .3 Consequences for experiments . . . . . . . . . . . . . . . . 90

6.3 Fate of the isotropic-nematic transition: the case $p=10$. . . . . . . 91

6.3 .1 Distribution of pseudo transition temperatures . . . . . . . . . 92

6.3 .2 Violation of hyperscaling . . . . . . . . . . . . . . . . 93

6.4 Summary $\ldots \ldots \ldots \ldots \ldots$. . . . . . . . . . . . . . . . . . . . 95 


\section{List of Figures}

$1.1 \quad$ Liquid crystal phases $\ldots \ldots \ldots$. . . . . . . . . . . . . . . . . . . 3

1.2 8CB molecule . . . . . . . . . . . . . . . . . . . . . . . . 5

$1.3 \quad 8$ CB bulk isotropic-nematic phase transition . . . . . . . . . . . . 6

1.4 Tobacco mosaic virus particles . . . . . . . . . . . . . . . . . . . . 7

1.5 Liquid-ordered and liquid-disordered bilayers . . . . . . . . . . . . . . 8

1.6 Nematic and perpendicular nematic schematic . . . . . . . . . . . . . 10

1.7 Landau-de Gennes free energy . . . . . . . . . . . . . . . . . . . . . . 14

1.8 Maier-Saupe theory distribution function . . . . . . . . . . . . . . . . 16

1.9 Maier-Saupe free energy $\ldots \ldots \ldots \ldots$. . . . . . . . . . . . . . . . . . . .

$2.1 \quad$ First-order phase transition simulation snapshots $\ldots . . . . . . . .26$

$2.2 \quad$ Simulation bimodal distribution $\ldots \ldots \ldots$. . . . . . . . . . . . . 28

2.3 successive umbrella sampling energy range schematic . . . . . . . . . 32

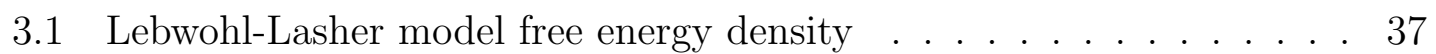

3.2 Pair interaction . . . . . . . . . . . . . . . . . . . . 38

3.3 Effect of the pair interaction . . . . . . . . . . . . . . . . . 39

4.1 Specific heat shifts . . . . . . . . . . . . . . . . . . . . . 43

4.2 Phase coexistence: energy density . . . . . . . . . . . . . . . . . . . . . . . . . . . . . . . . 45

$4.3 \quad$ Phase coexistence: simulation snapshot . . . . . . . . . . . . . . . . . 47

$4.4 \quad$ First-order isotropic-nematic transition in 3D . . . . . . . . . . . . . 48

4.5 First-order isotropic-nematic transition in 2D . . . . . . . . . . . . . . 49

4.6 Specific heat extrapolation . . . . . . . . . . . . . . . . . . . . 50

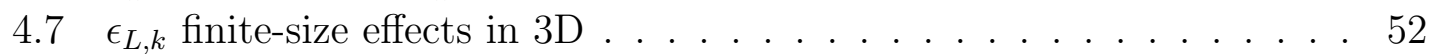

$4.8 \epsilon_{L, k}$ finite-size effects in 2D . . . . . . . . . . . . . . . . . 53

$4.9 \epsilon_{L, k}$ extrapolation $\ldots \ldots \ldots \ldots \ldots \ldots \ldots$

$4.10 \epsilon_{L, k}$ scaling. . . . . . . . . . . . . . . . . . . . . 55

$4.11 \epsilon_{L, k}$ extrapolation: more efficient method . . . . . . . . . . . . . 56

4.12 Latent heat extrapolation $\ldots \ldots \ldots$. . . . . . . . . . . 56

5.1 Phase coexistence of $8 \mathrm{CB} \quad \ldots \ldots \ldots \ldots$. . . . . . . . . . . . . . . . . . . . 60

5.2 Simulation setup schematic . . . . . . . . . . . . . . . . . 61

5.3 2D: no phase transition . . . . . . . . . . . . . . . . . . . . 63

5.4 2D: continuous phase transition . . . . . . . . . . . . . 66

5.5 2D: first-order phase transition $\ldots \ldots \ldots$. . . . . . . . . 67 
5.6 Thin film: continuous phase transition . . . . . . . . . . . . . . . 69

5.7 Thin films: towards a first-order phase transition . . . . . . . . . . 71

$5.8 \quad$ Capillary phase diagram with $p=8 \ldots \ldots$. . . . . . . . . 73

$5.9 \quad$ Capillary phase diagram with $p=20$. . . . . . . . . . . . . . . 73

5.10 Stretched simulation boxes . . . . . . . . . . . . . . . . . . 75

5.11 Kelvin equation . . . . . . . . . . . . . . . . . . . . 77

$6.1 \quad$ Liquid crystal to aerogel pinning . . . . . . . . . . . . . . . . . 80

6.2 Silica aerogel TEM micrographs . . . . . . . . . . . . . . . . . 80

6.3 $\quad$ Excess heat capacity of isotropic-nematic transition of 8CB in aerogel 81

6.4 Effect of quenched disorder . . . . . . . . . . . . . . . . 84

6.5 Violation of self-averaging . . . . . . . . . . . . . . . . . . . 86

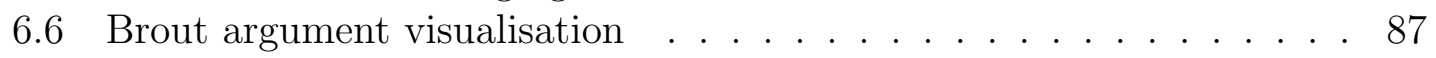

6.7 Connected and disconnected correlation functions . . . . . . . . . . . 88

6.8 Evidence for no transition . . . . . . . . . . . . . . . . . . . . . . 89

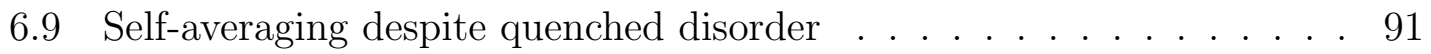

6.10 Evidence for a phase transition . . . . . . . . . . . . . . . . . . . 92

6.11 Thermal and disorder cumulants . . . . . . . . . . . . . . . . . . . . . 94 


\section{An introduction to liquid crystals}

\section{Contents}

\begin{tabular}{|c|c|}
\hline$\cdots \cdots \cdots \cdots \cdots \cdots \cdots$ & 1 \\
\hline$\ldots \ldots \ldots \ldots$ & 2 \\
\hline $1.2 .1 \quad$ Isotropic and nematic phases & 2 \\
\hline $1.2 .2 \quad$ Smectic phases & 4 \\
\hline 1.2 .3 Inducing phase transitions: thermotropic versus lyotropic & \\
\hline systems $\ldots \ldots \ldots \ldots \ldots \ldots \ldots \ldots$ & 4 \\
\hline$\ldots \ldots \ldots \ldots \ldots$ & 4 \\
\hline 1.3.1 Small organic molecules & 5 \\
\hline 1.3.2 Long helical rods & 6 \\
\hline $1.3 .3 \quad$ Amphiphilic compounds & 7 \\
\hline $1.3 .4 \quad$ Elastomers & 7 \\
\hline 1.4 Isotropic-nematic transition: bulk theoretical approaches & 9 \\
\hline $1.4 .1 \quad$ Nematic order parameter & 9 \\
\hline 1.4.2 Landau-de Gennes phenomenological theory. & 13 \\
\hline $1.4 .3 \quad$ Maier-Saupe theory & 14 \\
\hline $1.4 .4 \quad$ Onsager theory & 16 \\
\hline Role of confinement: scope of the thesis . . . . . . . . . & 18 \\
\hline 1.5.1 Thesis outline. & 19 \\
\hline
\end{tabular}

\subsection{Motivation}

Despite first being discovered in 1888 by Friedrich Reinitzer when examining the properties of derivatives of cholesterol [1, it was only the late 1960s and 1970s which became the boom years for research in liquid crystals, as the usefulness of liquid crystal displays was envisaged. The success of these efforts is clear: it is difficult nowadays to buy a television or a computer which does not use a liquid crystal display. Liquid crystals are used in many other applications, such as in thermal sensors [2], as the lasing medium in liquid crystal lasers [3], as templates for the 


\section{An introduction to liquid crystals}

synthesis of inorganic nanostructures [4, 5, 6], and for electroluminescent diodes [7]. As a result of this research, there is a thorough understanding of the macroscopic bulk properties of liquid crystals as exhibited by numerous review books on the subject [8, 9]. Furthermore, de Gennes was awarded with the Nobel prize in physics in 1991 "for discovering that methods developed for studying order phenomena in simple systems can be generalized to more complex forms of matter, in particular to liquid crystals and polymers" [10].

Despite this success, it is fair to say that our understanding of liquid crystals in the presence of confinement is less developed compared to that in bulk [11]. To be precise, by confinement we explicitly mean the behavior of liquid crystals in the presence of surfaces or substrates (which is, in fact, the "natural habitat" for most applications). The discovery of polymer dispersed liquid crystals in the mid-1980s (by phase separation [12] and emulsification [13, 14]), and their suitability for displays heralded a new beginning of research in confined liquid crystals. However, because of the increase in complexity of liquid crystal phase behavior induced by confinement, there is still strong, active research in this field [15]. Put simply: confinement of liquid crystals is common, but full understanding of the role of confinement is still lacking.

The aim of this thesis is to further contribute to the fundamental understanding of how liquid crystal phase behavior, in particular the isotropic-to-nematic transition, is affected by the presence of confining surfaces. To this end we use the Monte Carlo simulation method which has been shown, in recent years, to be well suited to study problems of this kind [16, 17].

\subsection{Liquid crystal basics}

\subsubsection{Isotropic and nematic phases}

Liquid crystals take after their name; they can display both disorder (characteristic of a liquid) and order (characteristic of a crystal). The liquid crystal phase in which any kind of order is completely absent is called the isotropic phase, see Fig. 1.1(a). What is depicted is an ensemble of molecules whose positions and orientations are randomly distributed. Consider now the ensemble shown in Fig. 1.1(b). The positions of the molecules are still random, but now there is preferred alignment of the molecules along some axes. The phase shown in Fig. 1.1(b) is called the nematic phase; it is the liquid crystal phase showing the least amount of order possible (namely orientational order only).

The nematic phase is the one we focus on in subsequent chapters. The essential properties of the nematic are as follows:

1. There is no long-range order in the center-of-mass positions of the individual molecules. Therefore, the X-ray diffraction patterns exhibit no Bragg peak (unlike in a true crystal). 
(a)

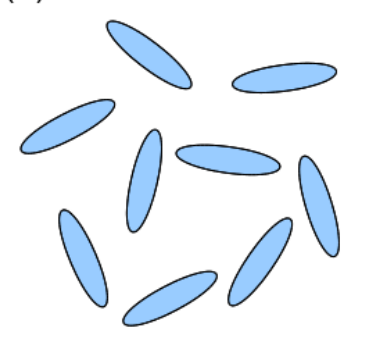

(c)

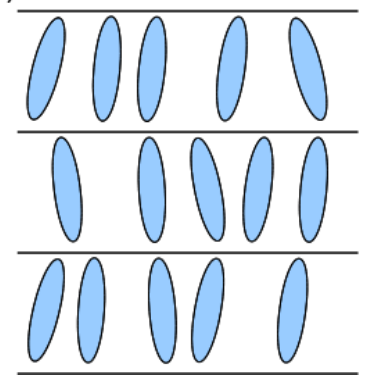

(b)

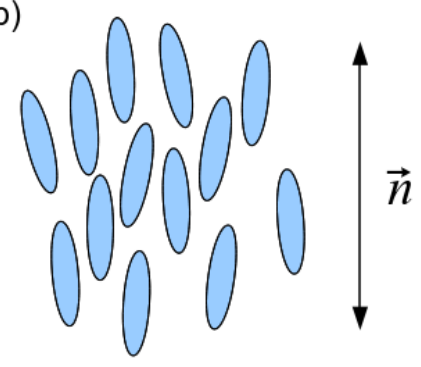

(d)

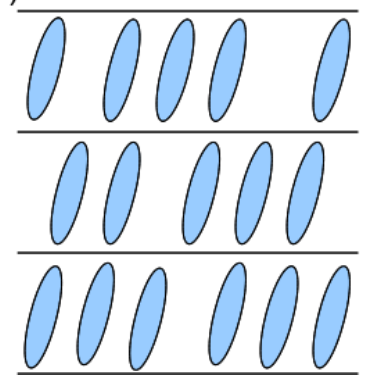

Figure 1.1: Schematic arrangement of molecules in various liquid crystalline phases in increasing order: (a) isotropic phase, (b) nematic phase, (c) smectic A phase, and (d) smectic C phase. In the isotropic phase there is no order. In the nematic phase there is long-range orientational order in average in the direction of the director $\vec{n}$ but no positional order. Smectic A phases have regular interlayer spacing, resulting in additional positional order in the direction of $\vec{n}$. Smectic C phases are further orientationally ordered, with particles oriented at a common tilt within the layers.

2. In the nematic phase the molecules preferentially align in a common direction called the director, and symbolized by $\vec{n}$ in what follows. Here $\vec{n}$ is a $d$ dimensional unit vector, as shown in Fig. 1.1(b). In this thesis we consider the experimentally relevant cases $d=2,3$ only.

3. We assume that $\vec{n}$ and $-\vec{n}$ are indistinguishable. This "inversion symmetry" of the nematic phase plays a major role in defining the order parameter.

In bulk systems, i.e. in the absence of any external fields and surfaces, the properties of the nematic phase do not depend on the direction in which $\vec{n}$ is pointing. However, this ideal situation of complete rotational invariance may be difficult to achieve in experiments because confining walls are always present, which typically force a certain direction for $\vec{n}$ (anchoring effects [18]). In addition, in simulations, it may be that the commonly used periodic boundary conditions induce preferred 
directions (so-called "easy-axes" [19]). Nematic phases are typically uniaxial; light propagates only in one direction through the liquid crystal. Recently, however, it has been shown that this need not necessarily be the case. Biaxial nematics, where light propagates in two directions through the liquid crystal were, in fact, synthesized in 2004 [20].

\subsubsection{Smectic phases}

Of course, the nematic phase is not the only structure that can arise: depending on the type of liquid crystal and the conditions (temperature, density, chemical environment) it is under, different structures (phases) may arise. For example, if the molecules also display some positional order, they give rise to phases known as smectics. Smectics, in general, are layered structures with well-defined inter-layer spacing. This spacing can be measured by X-ray diffraction, similar to crystals. Original work by G. Friedel only recognized one type of smectic liquid crystal [21], now known as smectic A, shown in Fig. 1.1(c). Smectic A has regular inter-layer spacing but irregular spacing within each layer. In fact, within each layer the molecules show no long-range positional order and they behave like a two-dimensional liquid. However, individual molecules tend to point in the same direction, perpendicular to the plane of the layers. Other smectics display variations on this basic theme. For example, smectic $\mathrm{C}$ is identical to smectic A except that individual molecules are positioned at a common tilt within each layer, see Fig. 1.1(d). For this reason, smectic C is optically biaxial whereas smectic A is optically uniaxial.

\subsubsection{Inducing phase transitions: thermotropic versus lyotropic systems}

In addition to the structure of the phases that liquid crystals can form, we can characterize liquid crystals by how they respond to external conditions. The most commonly used distinctions are the thermotropic liquid crystals, which respond to changes in temperature, and the lyotropic liquid crystals, which respond to changes in the overall density. In a thermotropic bulk liquid crystal, for example, one can induce a phase transition from the isotropic to the nematic phase by decreasing the temperature. By decreasing the temperature further, the nematic phase can undergo a transition to the smectic phase [22. Note that a "hard" distinction between thermotropic and lyotropic behavior is not always feasible, as some liquid crystals display thermotropic and lyotropic characteristics simultaneously [23].

\subsection{Liquid crystal molecules}

An obvious prerequisite for any of the ordered phases shown in Fig. 1.1 is that the molecules, in addition to a center-of-mass coordinate, must also have some "sense 


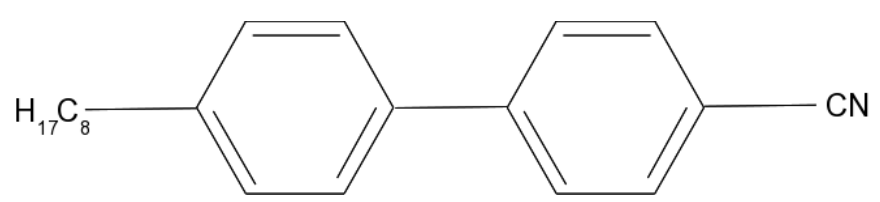

Figure 1.2: The chemical structure of the $8 \mathrm{CB}$ molecule, one of the most commonly used molecules to study liquid crystal phase transitions. The "related" molecules $5 \mathrm{CB}, 6 \mathrm{CB}$, and $7 \mathrm{CB}$ have shorter $C_{n} H_{2 n+1}$-side chains.

of direction". That is, the individual molecules must be spatially anisotropic. Common constituent molecules are organic molecules, helical rods (both found in nature or artificially made), or more complicated structures consisting of polymer chains. Brief descriptions of each are given next.

\subsubsection{Small organic molecules}

These small organic molecules are typically of size $\sim 10 \AA$ long and $\sim 1 \AA$ in diameter. Some examples are PAA, MBBA, and the CB family of molecules. The 8CB molecule, for example, consists of two aromatic rings connected to a $\mathrm{CN}$ tail and longer side-chain, as shown in Fig. 1.2. PAA is found in a nematic state at $389 \mathrm{~K}-408 \mathrm{~K}$ [24], whereas MBBA is found in a nematic state at $\sim 293 \mathrm{~K}-320 \mathrm{~K}$ [25], making it more suitable for experiments. The nematic state is also found at approximate room temperatures for the CB molecules [26]. All of these molecules are relatively long (compared to their width) and rigid. It is typically easiest to induce a phase transition in pure systems of such molecules by varying temperature; therefore, these systems are thermotropic.

The liquid crystal 8CB, shown in Fig. 1.2, has been studied extensively in bulk [28], in (effectively) two dimensions (via confinement between parallel substrates) [29, 30, 31], as well as in other geometries such as cylindrical pores [32, and its behavior is generally well-known in such environments. The $8 \mathrm{CB}$ molecules change phase thermotropically, and therefore the phase transitions are defined as occurring at certain temperatures. In bulk, $8 \mathrm{CB}$ exhibits transitions between liquid crystal phases with increasing temperature from the smectic-A phase (SmA), to the nematic phase $(\mathrm{N})$, to the isotropic phase (I) [28, 33, 34, 35], with transition temperatures as:

$$
\mathrm{SmA} \stackrel{306.8 \mathrm{~K}}{\longrightarrow} \mathrm{N} \stackrel{313.6 \mathrm{~K}}{\longrightarrow} \mathrm{I} .
$$

In $d=3$ dimensions, the smectic A - nematic phase transition is continuous, while the nematic-to-isotropic (IN) transition is weakly first-order. The fact that the latter transition is weakly first-order is manifested by small discontinuities in density, specific heat, and birefringence (for example, the volume change in $8 \mathrm{CB}$ samples at the IN transition is only $0.35 \%$ [36]). In Fig. 1.3 we provide experimental data of the 
1 An introduction to liquid crystals

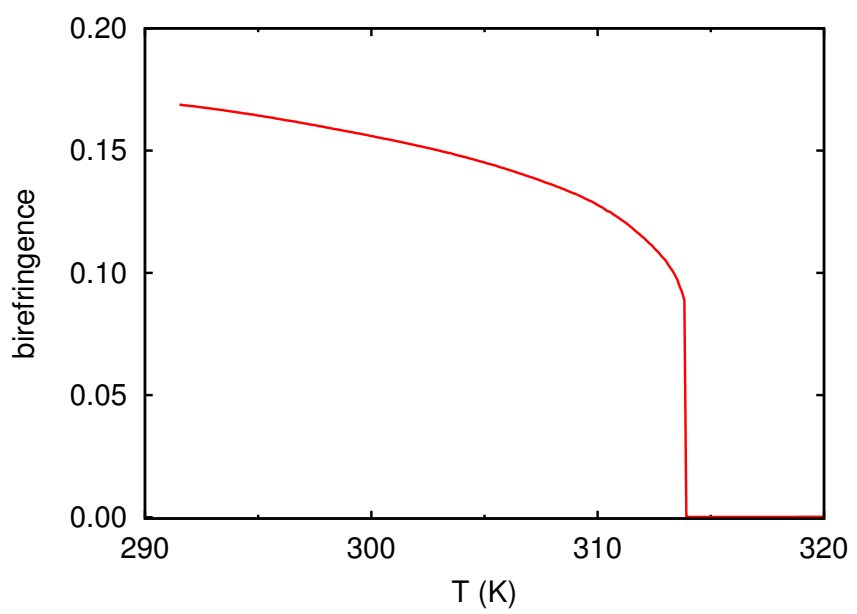

Figure 1.3: Experimental data of the IN phase transition of the liquid crystal 8CB in three-dimensional bulk. The birefringence, which can be thought of as a measure of nematic order [27], is shown versus temperature in Kelvin. At $\sim 313.7 \mathrm{~K}$ we observe the characteristic "jump" corresponding to the first-order transition from the isotropic (zero birefringence) to the nematic (finite birefringence) phase. Data kindly contributed by Patrick Huber and Andriy Kityk.

IN phase transition of the liquid crystal $8 \mathrm{CB}$ in bulk, where the birefringence is measured versus temperature. The discontinuous jump between the high-temperature isotropic phase and low-temperature nematic phase takes place at $\sim 313.7 \mathrm{~K}$.

\subsubsection{Long helical rods}

Long helical rods are found in nature but are also easily constructed in the laboratory. For example, many polypeptides are rod-shaped and have lengths of $\sim 100 \AA$ and widths of $\sim 10 \AA$. When concentrated, these molecules can form liquid crystal phases. Similar behavior is found for DNA and some viruses, most famously the tobacco mosaic virus, which has length $\sim 3000 \AA$ and width $\sim 200 \AA$ [37. Such rods are particularly useful for experiments as they are of exactly the same size (monodisperse). Glass and plastic fibers can also be formed into similar rods, having larger size, but similar ratios of length to width. For all such rods a phase transition can be most easily induced by changing their densities; therefore, systems of such rods are lyotropic. An additional advantage of the relatively large particle size is the possibility of single particle visualization in experiments, see Fig. 1.4. 


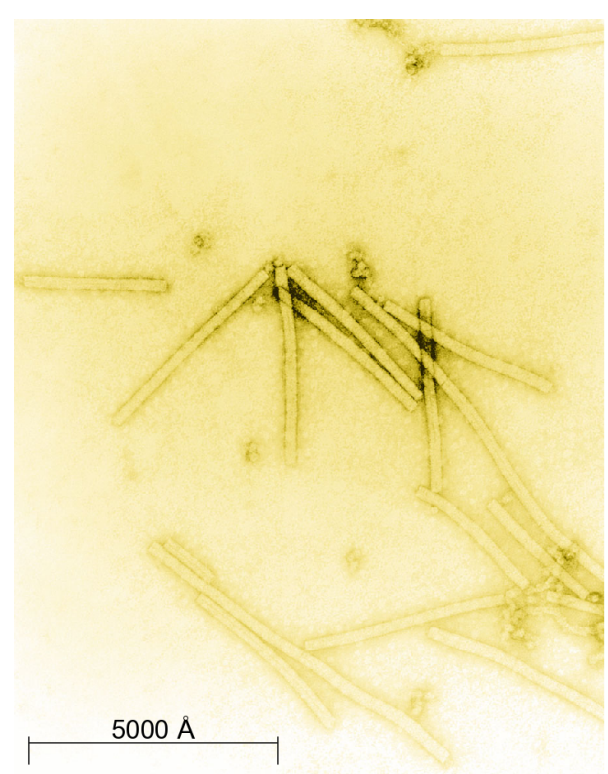

Figure 1.4: An electron micrograph of tobacco mosaic virus particles at 160,000x magnification. The particles are stained in order to enhance visibility. The particles are of uniform width and very similar length, making them attractive for experiments [38].

\subsubsection{Amphiphilic compounds}

Amphiphilic compounds, with polar heads (attracted to water) and apolar tails (avoiding water) can cluster together to become the building units of larger structures such as rods and sheets, which can yield liquid crystal phases [39]. These systems can be both lyotropic and thermotropic. A famous example is the main phase transition in membranes. In this case, the membrane undergoes a transition from a liquid-disordered to a liquid-ordered phase. In both phases, there is no positional order in the lipid centers-of-mass, but in the liquid-ordered phase the lipid tails are aligned, somewhat resembling a nematic phase [40, 41], as shown in Fig. 1.5.

\subsubsection{Elastomers}

Liquid crystal elastomers (LCEs) are formed by crosslinking a liquid-crystalline polymer melt [43]. If the crosslinking is performed with the polymer melt being in the isotropic phase, the low temperature phase of the LCE will be a polydomain structure [44, 45, 46]. Inside each domain, the sample is nematic in the sense that there is preferred alignment along the director, but the directors between different polydomains are randomly oriented from each other. Hence, there is no long-range nematic order. The reason we mention LCEs here is because the crosslinkers provide a kind of quenched disorder. The effect of quenched disorder on the isotropic-to-nematic 
1 An introduction to liquid crystals

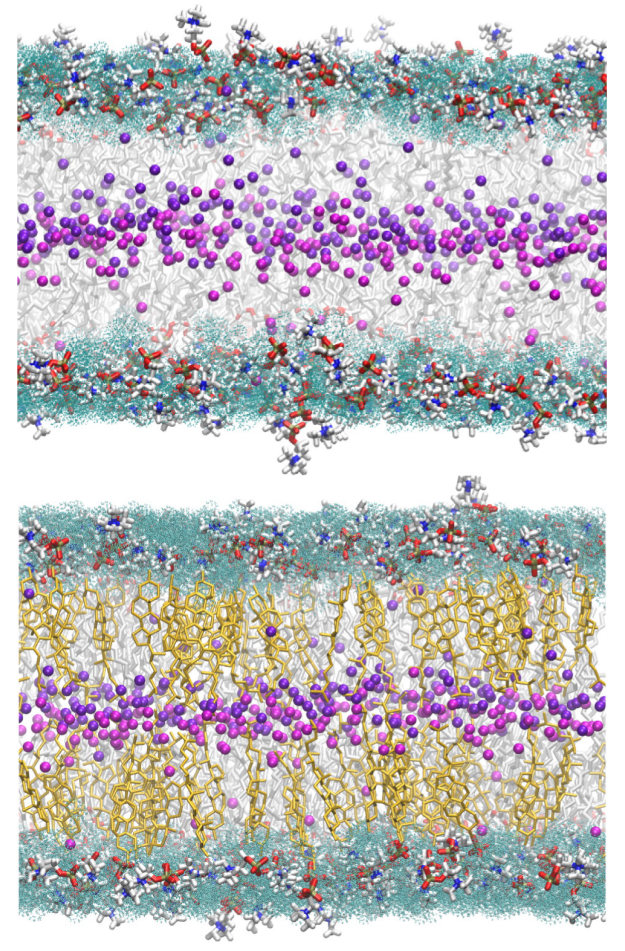

Figure 1.5: Simulation snapshots of liquid-disordered and liquid-ordered bilayers. The lipid molecules (grey and gold) form the cell membrane. The tails of the lipid molecules (purple) are much less ordered in the absence of cholesterol (top image) than when cholesterol is present (bottom image). The orientational order in the tail conformations somewhat resembles that of a nematic phase. Reprinted from Ref. [42] with permission from Elsevier. 
transition plays a major role in the final chapter of this thesis.

\subsection{Isotropic-nematic transition: bulk theoretical approaches}

From the experimental evidence, it transpires that the bulk isotropic-to-nematic section (in three dimensions) is (weakly) first-order. We now present a number of theories commonly used to describe this bulk transition. Common to all systems which undergo an isotropic-to-nematic phase transition in bulk, is that the phase transition is first-order and not continuous. We do not need statistical theories to understand why this must be the case. In the isotropic phase the system is rotationally invariant, while in the nematic phase this invariance is broken; the phase transition therefore must be first-order [27]. However, we do need statistical theories in order to understand the influence of the control parameters (temperature or otherwise) driving the transition. In this section, we discuss three such theories. The principal aspect these theories attempt to reproduce is the jump in order parameter at the phase transition, as exhibited in Fig. 1.3. We emphasize that these theories do not capture the behavior in the presence of confinement.

\subsubsection{Nematic order parameter}

The first ingredient of any theoretical description (and simulation) is some notion of an order parameter to distinguish between the isotropic and nematic phases. In Fig. 1.1(b) we introduced the director $\vec{n}$, giving in which direction molecules preferentially align. However, we have not defined a measure of how strongly aligned they are in that direction, nor have we shown how to calculate $\vec{n}$ given a set of molecular orientations. To this end, consider the arrangements of particles in the isotropic and nematic phases of Fig. 1.1. One might naively guess that a scalar order parameter suffices to distinguish between the isotropic and nematic phase. However, more complicated phases (such as biaxial nematics) exist and it turns out that a scalar is insufficient to fully describe the nature of nematic ordering. In fact, the nematic order parameter takes the form of a tensor.

\section{Definition of the orientational tensor}

The form of the tensor follows quite naturally when one tries to compute the nematic director $\vec{n}$ for a system of $i=1, \ldots, N$ "rod-like" molecules. The orientation of molecule $i$ is given by a normalized vector $\vec{d}_{i}=\left(x_{i}, y_{i}, z_{i}\right)$ so that $\left|\vec{d}_{i}\right|=1$. The director $\vec{n}$ is given by that vector for which the projection

$$
P(\vec{n})=\frac{1}{N} \sum_{i=1}^{N}\left(\vec{n} \cdot \vec{d}_{i}\right)^{2}
$$


1 An introduction to liquid crystals

(a)

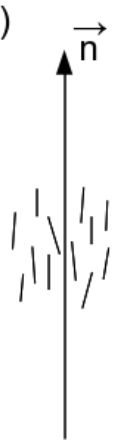

(b)

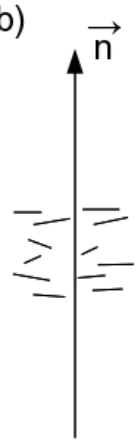

Figure 1.6: Schematic representation showing the difference in molecular order between (a) the "standard" nematic $(S>0)$ where particles align parallel to the director $\vec{n}$ and (b) the "perpendicular" nematic $(S<0)$ where the particles align $\perp$ to $\vec{n}$.

is maximized. Note the presence of the square in the definition of $P(\vec{n})$ which is required by the inversion symmetry, $\vec{d}_{i} \leftrightarrow-\vec{d}_{i}$, of the nematic phase. Finding the vector $\vec{n}$ that maximizes $P$ yields an Euler-Lagrange problem with constraint $|\vec{n}|=1$. This can be cast into an eigenvalue problem, $\mathbf{Q} \cdot \vec{n}=\lambda \vec{n}$, with eigenvalue $\lambda$, and where the tensor $\mathbf{Q}$ is given by

$$
\mathbf{Q}=\frac{3}{2}\left(\begin{array}{ccc}
\left\langle x^{2}\right\rangle-1 / 3 & \langle x y\rangle & \langle x z\rangle \\
\langle x y\rangle & \left\langle y^{2}\right\rangle-1 / 3 & \langle y z\rangle \\
\langle x z\rangle & \langle y z\rangle & \left\langle z^{2}\right\rangle-1 / 3
\end{array}\right)
$$

and where the angular brackets denote averages over all molecular orientations

$$
\langle\alpha \beta\rangle \equiv \frac{1}{N} \sum_{i=1}^{N} \alpha_{i} \beta_{i}, \quad(\alpha, \beta)=(x, y, z) .
$$

Note that the trace of $\mathbf{Q}$ is zero, since the molecular orientations are assumed to be normalized.

The ordering of nematic phases is thus encoded in the orientational tensor $\mathbf{Q}$ via its eigenvectors and eigenvalues. Note that $\mathbf{Q}$ can alternatively be expressed in its more usual "short form" as

$$
\mathbf{Q}_{\alpha \beta}=\frac{3}{2}\langle\alpha \beta\rangle-\frac{1}{2} \delta_{\alpha \beta}
$$

with Kronecker-delta $\delta_{\alpha \beta}$. In addition, we point out that the definition of $\mathbf{Q}$ in Eq.1.3 is very convenient from the point of view of simulations since the molecular orientations $\vec{d}_{i}$ are explicitly stored in memory. 


\section{Relation between the orientational tensor and nematic structure}

To distinguish between isotropic and nematic phases, one needs to compute $\mathbf{Q}$ and bring it into diagonal form. Since $\mathbf{Q}$ is symmetric it follows that all eigenvalues are real. Note also that the trace of $\mathbf{Q}$ is zero. In the isotropic phase, one trivially obtains a null matrix 1

$$
\mathrm{Q}_{\mathrm{iso}}^{\text {diag }}=\left(\begin{array}{lll}
0 & 0 & 0 \\
0 & 0 & 0 \\
0 & 0 & 0
\end{array}\right),
$$

while a (uniaxial) nematic phase yields

$$
\mathbf{Q}_{\text {nem }}^{\text {diag }}=S\left(\begin{array}{ccc}
-1 / 2 & 0 & 0 \\
0 & -1 / 2 & 0 \\
0 & 0 & 1
\end{array}\right), \quad S>0
$$

It is also possible to have a nematic phase where the particles align perpendicularly to the director, see Fig. 1.6. In that case one finds

$$
\mathbf{Q}_{\perp}^{\text {diag }}=S\left(\begin{array}{ccc}
-1 / 2 & 0 & 0 \\
0 & -1 / 2 & 0 \\
0 & 0 & 1
\end{array}\right), \quad S<0,
$$

i.e. the same as for the uniaxial nematic, but with a negative prefactor. Finally, for a biaxial nematic, one finds that

$$
\mathrm{Q}_{\text {biaxial }}^{\text {diag }}=\left(\begin{array}{ccc}
-S / 2-\eta & 0 & 0 \\
0 & -S / 2+\eta & 0 \\
0 & 0 & S
\end{array}\right),
$$

where $\eta$ measures the degree of biaxiality (when $\eta=0$ one recovers the nematic form of $\mathbf{Q}$ again).

\section{Scalar nematic order parameter}

From the above examples it follows that a scalar $S$ is sufficient to describe isotropic $(S=0)$, "standard" nematic $(S>0)$, and "perpendicular" nematic $(S<0)$ phases, but not biaxial nematics. Fortunately, biaxial nematics are relatively scarce, and we do not consider them in this thesis. Hence, we will mostly use the scalar $S$ as nematic order parameter in what follows. In simulations, this requires one to compute the eigenvalues of the orientational tensor $\mathrm{Q}$ : the signs of the eigenvalues tell us whether the phase is nematic, and the prefactor of $\mathbf{Q}$ tells us how strongly the nematic phase is aligned (see additional discussion in [47]).

\footnotetext{
${ }^{1}$ Of course, for the isotropic phase, diagonalization of $\mathbf{Q}$ is not necessary.
} 
Note that $S$ is related to the angular distribution function $f(\theta, \phi)$ via

$$
S=\int f(\theta, \phi)\left(\frac{3}{2} \cos ^{2} \theta-\frac{1}{2}\right) d \Omega
$$

where $f(\theta, \phi)$ denotes the probability of a molecule pointing in the direction $(\theta, \phi)$ where $0<\theta<\pi$ and $0<\phi<2 \pi$ are the "standard angles" of spherical coordinates. As $f(\theta, \phi)$ is a probability, we require a normalization condition

$$
\int f(\theta, \phi) d \Omega=\int_{0}^{2 \pi} d \phi \int_{0}^{\pi} d \theta \sin \theta f(\theta, \phi)=1
$$

Note that it is implicitly assumed in Eq.1.10 that the director corresponding to $f(\theta, \phi)$ is parallel to the $z$-axes.

\section{Measurement of the nematic order parameter in simulations}

In our computer simulations we have direct access to the molecular orientations: the order parameter $S$ can thus be calculated explicitly from the eigenvalues of the orientational tensor $\mathbf{Q}$. The nematic order parameter follows from the eigenvalues of the orientational tensor $\mathbf{Q}$, which involves the diagonalization of a $3 \times 3$ matrix. The tensor $\mathbf{Q}$ is trivially computed in simulations using Eq. (1.5) and the molecular

orientations $\vec{d}_{i}$. The diagonalization is performed using the exact expression for the roots of a cubic polynomial. Comparing to Eq.(1.7), there are several choices to extract $S$. The usual definition of $S$ is simply to take the largest eigenvalue of Q; the nematic director $\vec{n}$ is given by the corresponding eigenvector. However, in the isotropic phase, this choice leads to a finite-size artifact. If always the largest eigenvalue is taken, one also finds a finite nematic order parameter in the isotropic phase of order $\mathcal{O}\left(N^{-1 / 2}\right)$, with $N$ the total number of molecules [48]. We therefore also occasionally use an alternative definition by taking $S$ to be $-2 \times$ the middle eigenvalue of $\mathbf{Q}$. As can be seen from Eq.(1.7), this choice also constitutes a valid definition of $S$, which has the advantage of yielding $S=0$ when averaged in the isotropic phase.

\section{Measurement of the nematic order parameter in experiments}

In experiments the above method of calculating the nematic order parameter is usually not possible (except in systems of colloidal rods where real-space resolution is available [49]). There are two fundamentally different ways the nematic order parameter can be measured in experiments. One can measure the anisotropy of some macroscopic function, for example magnetically [50], electrically [51], or optically [51, and the only additional knowledge required is of the maximum possible magnitude of the function being measured, i.e. the anisotropy of the perfectly ordered nematic phase. Birefringence is a good example of such a measure. The most simple case involves materials with uniaxial anisotropy, where there is no symmetry 
between perpendicular planes. The material causes a beam of light to be split into two components, each traveling at different velocities, and thus having different refractive indices, dimensionless in value. Birefringence is measured as the difference between these refractive indices, and thus in an isotropic material we expect the birefringence to be zero [52]. A birefringence measurement of the liquid crystal 8CB in bulk is given in Fig. 1.3. In the isotropic phase light passes through the liquid crystal at equal velocities, regardless of the direction of travel. The nematic phase is however anisotropic, and non-zero birefringence is measured.

Some experimental techniques, such as Raman scattering and nuclear magnetic resonance, measure $S$ through the anisotropy of individual molecules rather than the bulk anisotropies of the liquid crystal [53]. Although it is impossible to measure the anisotropy of a single molecule by these techniques, the statistical averages (temporal and spatial) of the molecular anisotropy is possible to measure.

\subsubsection{Landau-de Gennes phenomenological theory}

Having defined the nematic order parameter, we can now discuss the theoretical frameworks most commonly used to describe the bulk isotropic-nematic transition. The first is the phenomenological Landau-de Gennes theory. The aim of this theory is to describe a thermotropic isotropic-nematic phase transition using only general symmetry considerations of the order parameter. Neglecting spatial fluctuations (i.e. the mean-field approximation) the free energy density is Taylor expanded in the order parameter up to fourth-order as

$$
F(\mathbf{Q}, T)=\sum_{n=1}^{4} A_{n}(T) \operatorname{Tr}\left(\mathbf{Q}^{n}\right),
$$

with $\mathrm{Q}$ the tensor order parameter of the nematic phase given by Eq.(1.7), expansion coefficients $A_{n}(T)$, and where $\operatorname{Tr}$ denotes a trace (the reason one uses the trace is because the free energy must be rotationally invariant). Note that the temperature dependence is contained in the expansion coefficients. Since $\mathbf{Q}$ is traceless it follows that the linear term vanishes $A_{1}=0$. The second observation is that $\mathbf{Q}$ and $-\mathbf{Q}$ describe different phases, as was shown schematically in Fig. 1.6. Hence, we must allow for a non-zero cubic term in the expansion $A_{3} \neq 0$ (which marks a strong departure from the Ising ferromagnet where odd terms are explicitly forbidden by symmetry). The free energy may thus be written as

$$
F(S, T)=\frac{1}{2} r S^{2}-w S^{3}+u S^{4},
$$

where we substituted Eq.(1.7), and with re-defined expansion coefficients $r, w, u$.

We may now attempt to describe the isotropic-nematic transition using the above form of $F$. At the transition temperature $T_{\mathrm{IN}}$ the free energy of the isotropic phase $(S=0)$ must equal that of the nematic phase $\left(S=S_{\text {nem }}>0\right)$ while both phases 
1 An introduction to liquid crystals

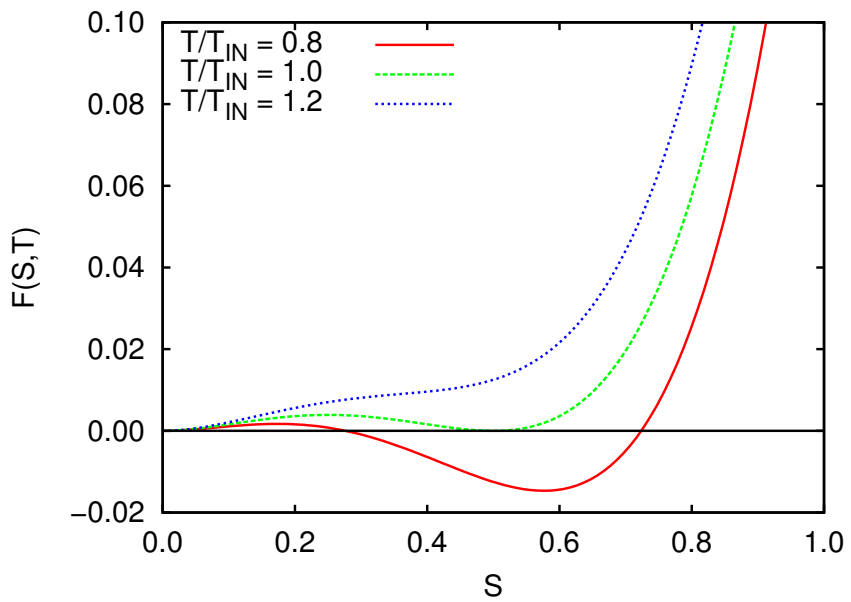

Figure 1.7: Generic shape of the free energy $F$ of Landau-de Gennes theory for $T / T_{I N}=0.8,1.0,1.2$ with coefficients $w=u=1$. The thick black line marks $F=0$. At $T>T_{I N}$ the isotropic phase is favored, while for $T<T_{I N}$ the nematic phase is favored. At $T=T_{I N}$ there is a first-order phase transition between the isotropic and nematic phases.

must correspond to a minimum in the free energy. This leads to the following set of equations

$$
\begin{gathered}
T=T_{\mathrm{IN}}: \quad \frac{1}{2} r S_{\mathrm{nem}}^{2}-w S_{\mathrm{nem}}^{3}+u S_{\mathrm{nem}}^{4}=0, \quad r S_{\mathrm{nem}}-3 w S_{\mathrm{nem}}^{2}+4 u S_{\mathrm{nem}}^{3}=(0.14) \\
r-6 w S_{\mathrm{nem}}+12 u S_{\mathrm{nem}}^{2}>0, \quad r>0
\end{gathered}
$$

which yield $r=w^{2} / 2 u$ and $S_{\text {nem }}=w / 2 u$ at the transition. The temperature dependence of the free energy around the transition is captured by writing $r=$ $\left(w^{2} T\right) /\left(2 u T_{\mathrm{IN}}\right)$ while $w, u$ are independent of temperature. The generic shape of $F$ is shown in Fig. 1.7 for $T / T_{\mathrm{IN}}=0.8,1.0,1.2$ and $w=u=1$. Due to the presence of the cubic term the transition is distinctly first-order.

The remarkable feat of Landau-de Gennes theory is that, purely based on the symmetry of the order parameter, it predicts a first-order isotropic-nematic transition. Indeed, this is the case in the majority of bulk experiments. The main drawback of the theory is, of course, its phenomenological nature: it is not a priori clear how $u$ and $w$ relate to material properties.

\subsubsection{Maier-Saupe theory}

The Maier-Saupe theory [54, 55, 56] provides a mean-field description of the isotropicnematic transition in thermotropic liquid crystals. The advantage over Landaude Gennes theory is that the transition temperature is expressed in terms of a 
material property, namely the Hamaker constant. In line with Landau-de Gennes theory, one writes the free energy $F$ as function of the nematic order parameter $S$

$$
F(S)=E(S)-T \Sigma(S)
$$

with energy $E$, orientational entropy $\Sigma$, and temperature $T$. Note that there is a competition between energy (high in the isotropic phase and low in the nematic phase) and entropy (low in the nematic phase and high in the isotropic phase) and this drives the isotropic-nematic transition. At low $T$ the system will minimize the energy and become nematic; at high $T$ the system will maximize its entropy and become isotropic. Consequently, at some temperature in-between an isotropicnematic transition must take place.

The order parameter $S$ in Maier-Saupe theory is the scalar definition of Eq.(1.10). For the energy one assumes that

$$
E(S)=-\frac{u S^{2}}{2}, \quad u>0,
$$

where the parameter $u$ is a material property expressing the strength of the interaction between neighboring molecules (which can be expressed in terms of the Hamaker constant). Hence, aligned phases have larger $S$ and are thus more energetically favorable. The entropy is given in terms of the angular distribution function $f(\theta, \phi)$ by the Boltzmann relation as

$$
\Sigma=-k_{B} \int f(\theta, \phi) \ln [\alpha f(\theta, \phi)] d \Omega,
$$

with $k_{B}$ the Boltzmann constant. As $f(\theta, \phi)=1 / 4 \pi$ in the isotropic, we choose $\alpha=4 \pi$ so that the entropy of the isotropic phase is zero. This results in a free energy of the form

$$
F_{\mathrm{MS}}(S)=-\frac{u S^{2}}{2}+k_{B} T \int f(\theta, \phi) \ln [4 \pi f(\theta, \phi)] d \Omega .
$$

However, to calculate $S$ and $\Sigma(S)$ fully, we require a distribution $f(\theta, \phi)$. The distribution is chosen to (1) yield an order parameter $S$, (2) be normalized, and (3) maximize the entropy. This leads to an Euler-Lagrange problem with two constraints which can easily be solved [57]. The solution takes the form $f(\theta, \phi) \propto \exp \left(\lambda \cos ^{2} \theta\right)$, with the Lagrange multiplier $\lambda$ setting the value of $S$, and where the proportionality constant follows from the normalization condition Eq. (1.11). Note that $\lambda<0$ describes a "perpendicular" nematic (see Fig. 1.6(b)), $\lambda=0$ an isotropic phase, and $\lambda>0$ a nematic phase, as seen in Fig. 1.8

For a given value $\lambda \geq 0$, one uses the corresponding $f(\theta, \phi)$ to compute the order parameter $S$ and entropy $\Sigma$, and uses these to calculate the free energy $F$ via Eq. (1.19). Any single value of $\lambda$ yields a single point on the curve $F(S)$; the full 


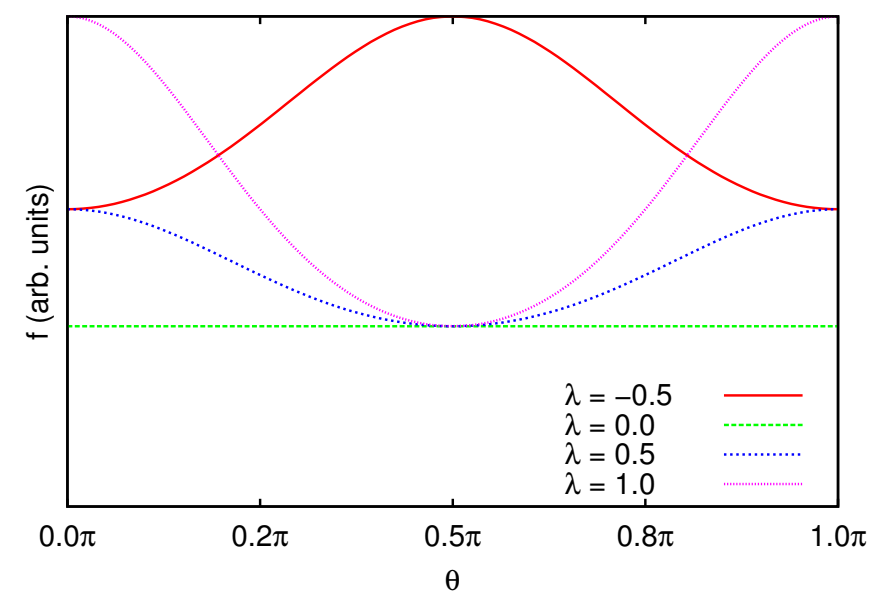

Figure 1.8: The angular distribution function $f(\theta, \phi)$ of Maier-Saupe theory for several values of the variational parameter $\lambda$. The value $\lambda=0$ corresponds to the isotropic phase (flat distribution). Positive values correspond to nematic phases (distribution peaks at $\theta=0, \pi$ ) while negative values correspond to "perpendicular nematics" (distribution peaks at $\theta=\pi / 2$ ).

curve is thus parametrized by $\lambda$. For small $u / k_{B} T$ there is a single minimum of the free energy at $S=0$, and the equilibrium state is therefore isotropic. However, at $u / k_{B} T \approx 4.55$ a second minimum of equal depth appears, which deepens as $u / k_{B} T$ increases. This describes the isotropic-nematic transition. There is a corresponding discontinuity in the order parameter at the transition, where it jumps from $S=0$ to $S \approx 0.44$, indicating a first-order phase transition, shown in Fig. 1.9. Following Eq. 1.17), this also implies a jump in the energy density $\Delta \rho / u=S^{2} / 2 \approx 0.1$.

Maier-Saupe theory is remarkably accurate [58], exhibiting good qualitative agreement with bulk experiments in general, in particular providing a very good estimate of the value of $S$ in the nematic phase at the phase transition [59]. Discrepancies with experiments are mainly due to the temperature dependence of $u$, which is ignored in the theory. The role of fluctuations in $S$ can also be important, but are also neglected in this mean-field treatment. Larger discrepancies with experiments are therefore expected in situations of confinement where fluctuations become particularly important.

\subsubsection{Onsager theory}

Onsager theory provides a description of the isotropic-nematic transition in lyotropic liquid crystals [60]. A very basic model of a lyotropic liquid crystal is a system of hard rods, modeled in three dimensions as cylinders with length $L$ and diameter $D$. We define the cylinder elongation as $\kappa=L / D$ and consider $\kappa \gg 1$ in what follows. 


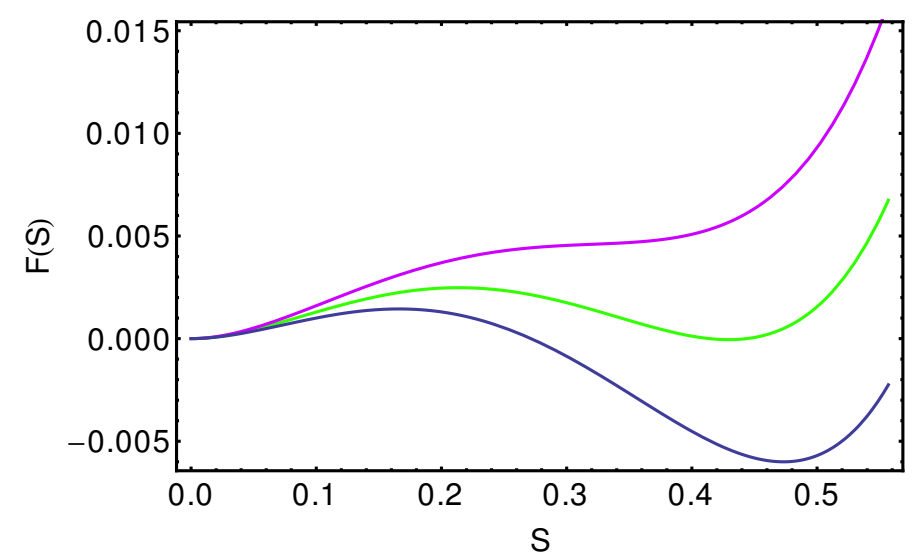

Figure 1.9: Free energy $F(S)$ of the Maier-Saupe model calculated for various values of order parameter $S$ at $u / k_{B} T=4.48,4.542,4.60$. For $u / k_{B} T=4.542$ (green curve) the two minima at $S=0$ (corresponding to the isotropic phase) and $S \approx 0.44$ (corresponding to the nematic phase) are of equal height, and the transition between the two phases is first-order. For $u / k_{B} T=4.48$ (pink curve) there is only one minimum and the transition is not first-order. For $u / k_{B} T>4.542$ the minimum at $S>0$ deepens.

The rods are hard meaning that they are not permitted to overlap, but otherwise the interaction energy is zero. The energy thus plays no role and the free energy is solely determined by the entropy. The isotropic-nematic phase transition is brought about because there are two competing entropy contributions: the orientational entropy $\Sigma_{\text {orient }}$ due to the rod orientations, and the translational entropy $\Sigma_{\text {trans }}$ due to the rod centers-of-mass. As such, the total entropy $\Sigma_{\text {tot }}$ is given by

$$
\Sigma_{\text {tot }}=\Sigma_{\text {orient }}+\Sigma_{\text {trans }}
$$

The orientational entropy may again be expressed in terms of the angular distribution function via Eq. 1.18). To obtain the translational entropy, we use the expression for an ideal gas, but with the volume $V$ replaced by $V-B$, where $B$ denotes the excluded volume (we thus assume that the rod concentration is low). The result is

$$
\Sigma_{\text {trans }} \approx k_{B} \ln \left(\frac{V}{N \Lambda^{3}}\right)-\frac{k_{B} B}{V}, \quad B \ll V,
$$

with $\Lambda$ the thermal wavelength, and $N$ the total number of rods in the system. Restricting ourselves to pair interactions, the excluded volume can be written as

$$
B \approx 2 D L^{2} N\langle|\sin \gamma|\rangle, \quad \kappa \gg 1
$$


where $\gamma$ is the angle between two rods, and where the average is computed as a double-integral over the angular distribution function

$$
\langle|\sin \gamma|\rangle=1 / 2 \iint f(\theta, \phi)|\sin \gamma| f\left(\theta^{\prime}, \phi^{\prime}\right) d \Omega d \Omega^{\prime} .
$$

This leads to a free energy of the form

$$
\frac{F_{\text {Onsager }}}{k_{B} T} \approx \Gamma\langle|\sin \gamma|\rangle+\int f(\theta, \phi) \ln [4 \pi f(\theta, \phi)] d \Omega,
$$

where additive constants have been dropped, $\Gamma=8 \kappa \eta / \pi$, and with the rod packing fraction defined as $\eta=\pi D^{2} L N /(4 V)$. To calculate the free energy explicitly an "Ansatz" for the angular distribution function is made

$$
f_{\lambda}(\theta, \phi)=\frac{\lambda}{4 \pi} \frac{\cosh (\lambda \cos \theta)}{\sinh \lambda}
$$

with variational parameter $\lambda>0$. Note that $\lambda \rightarrow 0$ corresponds to the isotropic phase, while $\lambda>0$ describes a nematic phase. If the free energy is calculated as function of the scalar nematic order parameter $S$ using $\Gamma \sim 9.4$, one again observes two minima at equal height characteristic of a first-order transition: one minimum at $S=0$ corresponding to the isotropic phase, and the other at $S \sim 0.84$ corresponding to the nematic phase [57]. The term $\Gamma$ in Eq. (1.24) thus plays a role analogous to $u / k_{B} T$ in Maier-Saupe theory. Note that $S$ in the nematic phase is considerably larger than observed in experiments, where typically $S \sim 0.4$ [61]. The Onsager model also has other faults; the change in density at the phase transition, for instance, is also much larger than observed in experiments 61].

\subsection{Role of confinement: scope of the thesis}

We have seen that nematics in bulk consist of anisotropic molecules aligned in a common direction called the director. We have shown that in a nematic phase the (diagonalized) orientational tensor $\mathbf{Q}$ takes the form of Eq.(1.7) with nematic order parameter $S>0$. In addition, we have presented a number of mean-field treatments of the isotropic-nematic transition, all showing that the isotropic-nematic transition is first-order.

While in bulk the use of mean-field approaches is usually safe, quite the reverse is true for a system in confinement. By confining a system (for example between two parallel walls) the dimension of the system is reduced. In these situations the assumption that the order parameter is uniform is no longer valid. For a correct description of the isotropic-nematic transition we now require a method whereby spatial fluctuations of the order parameter are included. The method of choice in this thesis are computer simulations. Computer simulations are, by nature of storing information of all constituent particles, ideal to investigate such effects. 
The situation of liquid crystals confined between parallel walls is covered in chapter 5. This is presumably the most simple case of confinement conceivable but the results are already surprising. While we generally observe the loss of long-range nematic order as the distance between the walls becomes smaller, owing to the Mermin-Wagner theorem [62], we nevertheless observe an isotropic-nematic transition. The striking result is that the corresponding transition can be continuous, as well as first-order, depending on the details of the interaction potential. The fact that both continuous and first-order transitions can occur appears consistent with experiments [29, 30, 31] as well as with a recent mathematical proof that such non-universal behavior is indeed allowed 63 .

In chapter 6 we consider a far more complex form of confinement, namely of a liquid crystal inside a porous medium. In this case, the confinement constitutes a form of quenched disorder. In addition to spatial fluctuations in the order parameter, one now also needs to consider the fluctuation of results between different samples of the quenched disorder. To tackle this problem analytically is extremely difficult, but much less so in simulations. The main finding of chapter 6 is that fluctuations between disorder samples are very pronounced, and need to be carefully measured if a consistent description of the isotropic-nematic transition is to be obtained.

\subsubsection{Thesis outline}

In the next chapter we give an overview of the simulation methods used throughout this thesis. Following that, in chapter 3 we introduce the Lebwohl-Lasher model, which is one of the standard models used in computer simulations to describe thermotropic liquid crystals, and which is used as a basis throughout this thesis. In chapter 4 we describe finite-size effects at first-order phase transitions. We present results dealing with planar confinement in chapter 5 and porous media in chapter 6. Our results have also been published in the following peer-review journals:

1. J.M. Fish and R.L.C. Vink, Finite-size effects at first-order isotropic-to-nematic transitions, Phys. Rev. B 80, 014107 (2009);

2. J.M. Fish and R.L.C. Vink, Isotropic-to-nematic transition in confined liquid crystals: An essentially nonuniversal phenomenon, Rhys. Rev. E 81, 021705 (2010); and

3. J.M. Fish and R.L.C. Vink, Nematics with quenched disorder: violation of self-averaging, Phys. Rev. Lett. 105, 147801 (2010). 
1 An introduction to liquid crystals 


\section{Simulation methods}

\section{Contents}

\begin{tabular}{|c|c|c|}
\hline 2.1 & Introduction $\ldots \ldots \ldots \ldots \ldots \ldots \ldots$ & 21 \\
\hline 2.2 & The Metropolis algorithm $\ldots \ldots \ldots \ldots \ldots$ & 22 \\
\hline & 2.2.1 Thermal equilibrium: Boltzmann distribution . . & 22 \\
\hline & $2.2 .2 \quad$ Importance sampling . . . . . . . . . . . . . . & 23 \\
\hline & 2.2.3 Markov chain Monte Carlo: detailed balance and ergodicity & 23 \\
\hline & 2.2.4 $\quad$ Metropolis algorithm & 24 \\
\hline 2.3 & Exponential slowing down & 25 \\
\hline 2.4 & Biased sampling algorithms & 27 \\
\hline & 2.4.1 Wang-Landau algorithm & 27 \\
\hline & 2.4.2 Transmission matrix Wang-Landau algorithm . . . & 29 \\
\hline & $2.4 .3 \quad$ Successive umbrella sampling . . . . . . . . . . . . & 32 \\
\hline
\end{tabular}

\subsection{Introduction}

The aim of our computer simulations is to describe the equilibrium phase behavior of a large number of liquid crystal molecules interacting via the Lebwohl-Lasher Hamiltonian (to be discussed in detail in chapter 3). The Monte Carlo method is well suited to probe thermal equilibrium properties and will therefore be our method of choice. In fact, early simulations of the Lebwohl-Lasher model also used Monte Carlo methods [64, 65] and performing such simulations using single spin dynamics with "standard" Metropolis sampling is straightforward. However, this standard method is inefficient near a first-order phase transition due to the formation of interfaces (these cost free energy which in turn leads to exponential slowing down making the simulation very inefficient [66]). The simulations of this thesis are therefore based on more sophisticated algorithms, such as Wang-Landau [67, 68, 65] and successive umbrella sampling [69]. In addition, we use the transition matrix to also extract information from Monte Carlo moves that are rejected.

A further point to note is that ideally we wish to describe the thermodynamic limit, where the number of molecules tends to infinity while the density remains constant. However, the thermodynamic limit cannot be simulated directly on a 


\section{Simulation methods}

computer (computer memory is finite) and so an additional important ingredient of this work is the use of finite-size scaling. The application of finite-size scaling to continuous spin systems with quenched disorder constitutes an important aspect of the new results of this thesis, and is therefore not presented in this chapter but instead in the separate results chapters, namely chapters 4, 5, and 6.

In this chapter we describe the Monte Carlo methods used in this thesis. We begin with a discussion of the "standard" Metropolis algorithm applied to the LebwohlLasher model. We then discuss the limitations of this algorithm near a first-order phase transition which brings us to the discussion of Wang-Landau and successive umbrella sampling (including a description of how Wang-Landau sampling can be made even more efficient by using information from rejected Monte Carlo moves via the transition matrix).

\subsection{The Metropolis algorithm}

The general method of performing a Monte Carlo simulation is well known. A change to the system is proposed (via a Monte Carlo move) which is then accepted with a certain probability. The idea is to generate a sequence of states "somewhat representative" of a real system, for example an experiment. Fluctuations move an experiment from state to state as time progresses; a Monte Carlo simulation models this by also permitting these fluctuations to occur and calculates the probability of the system moving between the different states. Typically, as we can sample only a small fraction of total states, we need to try to make this sample as representative as possible of the whole system. For the state to occur with the appropriate probability we need to design rules governing how we change from one state to another.

\subsubsection{Thermal equilibrium: Boltzmann distribution}

At temperature $T$ a system in thermal equilibrium is found in state $\mu$ at energy $E_{\mu}$ with probability $p_{\mu}$ according to the Boltzmann distribution

$$
p_{\mu}=\frac{1}{Z} \exp \left(-\frac{E_{\mu}}{k_{B} T}\right),
$$

where the normalization constant $Z$ is called the partition function and has value

$$
Z=\sum_{\mu} \exp \left(-\frac{E_{\mu}}{k_{B} T}\right) .
$$

The expectation value $\langle Q\rangle$ of an observable $Q$, such as the order parameter or the energy is calculated as the weighted average

$$
\langle Q\rangle=\sum_{\mu} Q_{\mu} p_{\mu}
$$


where $Q_{\mu}$ is the value of the observable in state $\mu$ (in continuous systems the sum over all states is replaced by an integration over phase space).

\subsubsection{Importance sampling}

For sufficiently simple systems the summation over all states can be performed analytically (such as in the 1D Ising model). In that case an exact expression for $Z$ can be derived and expectation values can be computed by means of an appropriate derivative. In general, however, an exact summation is not possible; such is already the case for the (seemingly simple) Lebwohl-Lasher model. The idea of equilibrium Monte Carlo is to estimate expectation values by replacing the sum over all possible states with a sum over a subset $\left\{\mu_{1}, \mu_{2}, \ldots \mu_{M}\right\}$ of states. Assuming that the Monte Carlo scheme generates state $\mu_{i}$ with probability $g_{\mu_{i}}$ the best estimate of an expectation value may be written as

$$
\langle Q\rangle \approx \frac{1}{Z} \sum_{i=1}^{M} \frac{1}{g_{\mu_{i}}} Q_{\mu_{i}} \exp \left(-\frac{E_{\mu_{i}}}{k_{B} T}\right),
$$

with the partition sum approximated by

$$
Z \approx \sum_{i=1}^{M} \frac{1}{g_{\mu_{i}}} \exp \left(-\frac{E_{\mu_{i}}}{k_{B} T}\right) .
$$

Typically, only a small fraction of all states contribute greatly to the partition function. It is for this reason that selecting states with equal probabilities is a poor choice. Monte Carlo simulations therefore attempt to select states intelligently; this is called importance sampling. Since an experimental system samples states from a Boltzmann distribution, given by Eq.(2.1), it makes sense to do the same when simulating. If we manage to design an algorithm such that $g_{\mu_{i}} \propto \exp \left(-\frac{E_{\mu_{i}}}{k_{B} T}\right)$ the estimate of the expectation value simplifies to

$$
\langle Q\rangle=\frac{1}{M} \sum_{i=1}^{M} Q_{\mu_{i}} .
$$

With this optimal choice, each generated state contributes with equal weight to the expectation value.

\subsubsection{Markov chain Monte Carlo: detailed balance and ergodicity}

Despite knowing the optimal probability with which the states should be generated, we are still lacking a practical algorithm to achieve this. To this end we use a Markov 


\section{Simulation methods}

process. A Markov process changes the system from one state $\mu$ to another state $\nu$ by means of proposing a random change to the starting state $\mu$ - the random change is called a Monte Carlo move. Repeating this many times a Markov chain of states is formed. In order for the sequence of states $\mu_{i}$ in the chain to appear with the desired target probability $p_{\mu_{i}}$ we require the conditions of ergodicity and detailed balance. For the Markov process to be ergodic, it must be possible to reach all states possible from some arbitrary starting state by repeated applications of the Monte Carlo move. This does not mean that the new state has to be accessible in a single move, but that there must be some sequence of moves connecting the two. The condition of detailed balance reads as

$$
p_{\mu} P(\mu \rightarrow \nu)=p_{\nu} P(\nu \rightarrow \mu),
$$

where $p_{\mu}$ is the probability distribution that one wishes to sample, and where $P(\mu \rightarrow$ $\nu)$ is the probability that, being in state $\mu$, the next step of the Markov process yields state $\nu$.

\subsubsection{Metropolis algorithm}

With the conditions of detailed balance and ergodicity in mind one easily constructs a Monte Carlo algorithm that will generate "representative" states for the LebwohlLasher model, i.e. according to the Boltzmann distribution. The Monte Carlo move that we use to simulate the Lebwohl-Lasher model is the generalized spin flip which we describe in chapter 3 . To derive the probabilities with which the Monte Carlo moves should be accepted one "breaks-down" the probability $P(\mu \rightarrow \nu)$ of Eq.2.7 into proposition and acceptance parts

$$
P(\mu \rightarrow \nu)=g(\mu \rightarrow \nu) A(\mu \rightarrow \nu),
$$

where $g(\mu \rightarrow \nu)$ is the probability that the Monte Carlo move proposes state $\nu$ (starting from state $\mu$ ) and $A(\mu \rightarrow \nu)$ is the probability that the proposed move is accepted. Thus, we do not necessarily move to each state that is proposed. We can therefore rewrite Eq.(2.7) as

$$
\frac{A(\mu \rightarrow \nu)}{A(\nu \rightarrow \mu)}=\frac{g(\nu \rightarrow \mu)}{g(\mu \rightarrow \nu)} \exp \left(-\frac{\Delta E}{k_{B} T}\right)
$$

with $\Delta E=E_{\nu}-E_{\mu}$ the energy difference between the states, and where for the target probability the Boltzmann distribution was used. The proposition probabilities depend on the type of Monte Carlo move used. For the generalized spin flip used in this thesis, it holds that $g(\nu \rightarrow \mu)=g(\mu \rightarrow \nu$ ) (see chapter 3) so the ratio cancels from Eq. $(2.9)$. In what follows, we will drop the proposition probabilities from our notation.

Eq. (2.9) only gives the ratio between accept probabilities, and thus we still have some freedom in choosing the probabilities themselves. Since we are dealing with 
probabilities we require $0 \leq A \leq 1$. Ideally we want to accept as many moves as possible. Consider a move from state $\mu \rightarrow \nu$ : the optimal choice is to choose $A(\mu \rightarrow \nu)$ as close to unity as possible. Consider first the case whereby the r.h.s. of Eq. (2.9) is greater than unity. In that case we can safely choose $A(\mu \rightarrow \nu)=1$, since for that choice the reverse probability $A(\nu \rightarrow \mu)$ yields a number smaller than unity. Similarly for moves where the r.h.s. is smaller than unity one chooses $A(\nu \rightarrow \mu)=1$ implying that in these situations $A(\mu \rightarrow \nu)<1$, i.e. there will now also be rejection. The optimal choice for many applications is therefore to choose the accept probabilities as

$$
A(\mu \rightarrow \nu)=\min \left[1, \exp \left(-\frac{\Delta E}{k_{B} T}\right)\right]
$$

which is called the Metropolis algorithm [70. Once again we emphasize that the above equation only holds when the proposition probabilities are symmetric: $g(\nu \rightarrow \mu)=g(\mu \rightarrow \nu)$.

\subsection{Exponential slowing down}

While the standard Metropolis algorithm has given valuable insights into the phase behavior of the Lebwohl-Lasher model the algorithm has its limitations. To be described in detail in chapter 3, the Lebwohl-Lasher model in 3D undergoes a firstorder phase transition between the isotropic and nematic phases [64]. At a first-order phase transition one encounters phase coexistence, where patches of isotropic and nematic domains coexist with each other. Hence, there is a considerable amount of interface in the system which corresponds to a large cost in free energy owing to surface tension [71]. The standard Metropolis algorithm performs poorly in this case.

To see why this is consider the snapshots in Fig. 2.1 showing how a first-order phase transition takes place in a system with periodic boundary conditions. Although these are snapshots of the Lebwohl-Lasher model, the behavior is general in the sense that it applies to any first-order phase transition [72, 73] (but to be explicit we shall speak of the isotropic-nematic transition). Starting in the isotropic phase (a) a first-order isotropic-nematic transition typically begins as a nucleation event with the formation of a nematic droplet (b). As the transition progresses the droplet grows until it interacts with itself through the periodic boundaries, upon which a strip configuration is obtained (c). The isotropic and nematic phase are then separated by two interfaces that run perpendicular to one of the edges of the simulation box since this geometry minimizes the total interface length. In $d$ dimensions the total interface length (area in $d=3$ ) equals $2 L^{d-1}$, with $L$ the edge of the simulation box. As the fraction of the nematic phase increases one observes the formation and growth of an isotropic droplet (d), until finally the system is completely nematic (e). 


\section{Simulation methods}

(a)

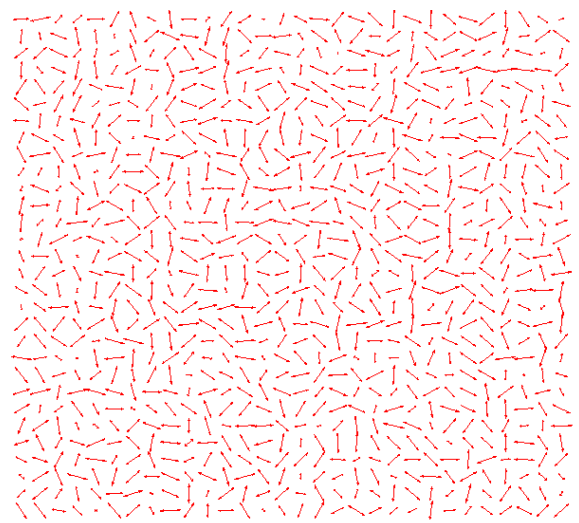

(b)

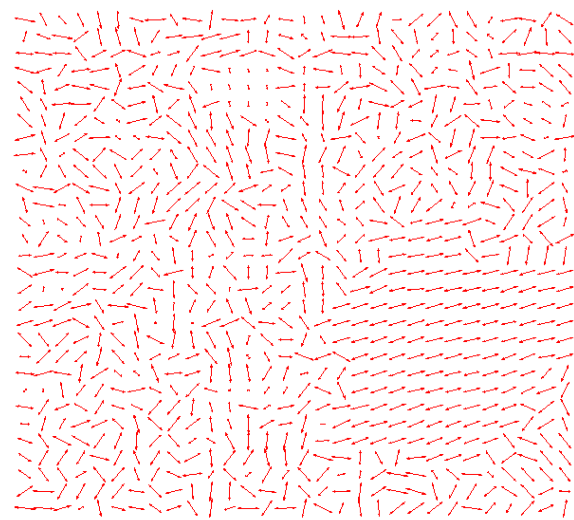

(c)

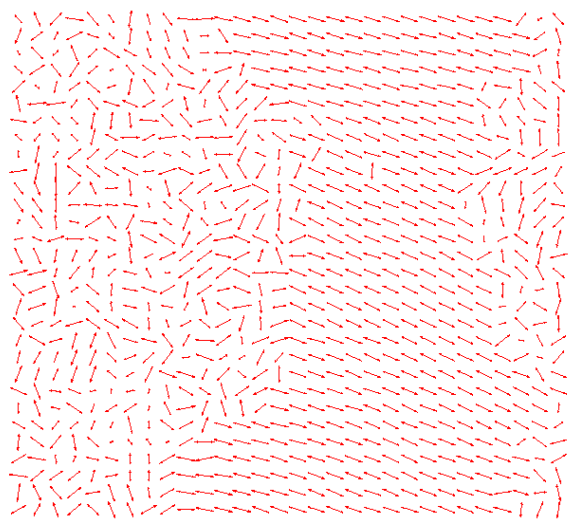

(d)

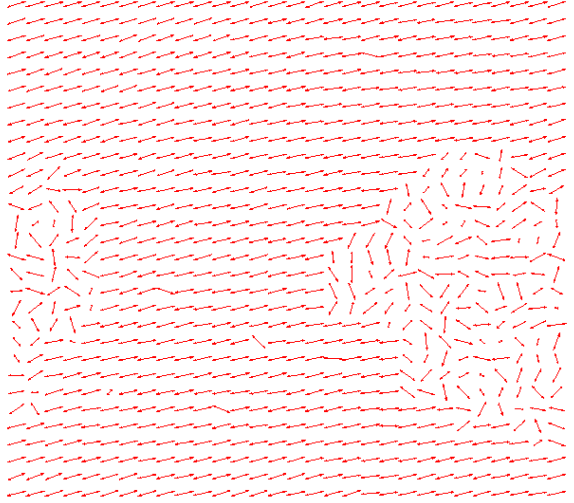

(e)

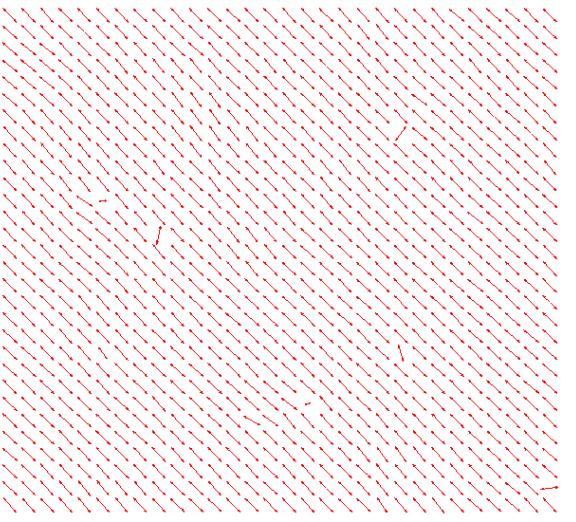

Figure 2.1: Simulation snapshots of a (generalized) Lebwohl-Lasher model on a twodimensional lattice exhibiting a first-order isotropic-nematic transition (note that the spins are three-dimensional). Starting in the isotropic phase (a) a droplet of the nematic is initially formed (b). The droplet spreads until it interacts with itself, because periodic boundary conditions are used, and it forms a strip configuration (c). The fraction of nematic phase increases until an isotropic phase is confined to a droplet (d), and finally the system becomes completely nematic (e). 
In a standard Metropolis Monte Carlo simulation with configurations appearing proportional to their Boltzmann weights, the strip configuration of Fig. 2.1(b) is extremely rare due to the large cost in free energy owing to interface tension. The corresponding free energy cost equals $\Delta F=2 \sigma L^{d-1}$, with $\sigma$ the interface tension, and thus $\Delta F$ grows rapidly with system size. An algorithm sampling from the Boltzmann distribution is therefore inefficient at a first-order phase transition. With such an algorithm, the CPU time required to observe the interface states grows exponentially with system size, hence the phrase exponential slowing down. For this reason we use the different Monte Carlo sampling strategies described in the following section.

\subsection{Biased sampling algorithms}

\subsubsection{Wang-Landau algorithm}

At a first-order isotropic-nematic phase transition in a thermotropic liquid crystal there is a difference between the phases not only in the nematic order parameter, but also in the energy. For example, in the theory of Maier-Saupe, the nematic order parameter "jumps" at the transition by an amount $\Delta S \approx 0.44$, which corresponds to a change in energy density $\Delta E / u \approx 0.44^{2} / 2$ (see section 1.4 .3 . The energy may therefore also be used as order parameter to distinguish between the isotropic and nematic phase.

To study the isotropic-nematic transition one could thus measure the distribution $P(E)$ defined as the probability to observe the system in a state with energy $E$ during the Monte Carlo simulation. At the isotropic-nematic transition, this distribution becomes bimodal: the peak at low energy corresponds to the nematic phase, the peak at high energy to the isotropic phase, and the region between the peaks to phase coexistence (see Fig. 2.2). However, as was explained in the previous section, if one samples from the Boltzmann distribution the coexistence region is rarely visited, and the bimodal structure of $P(E)$ is not observed (instead, only the isotropic or nematic peak is observed, depending on starting conditions).

To observe the full distribution we can use Wang-Landau sampling whereby a state $\mu$ does not appear with its Boltzmann weight, but instead with a probability given by

$$
p_{\mu} \propto \frac{1}{G\left(E_{\mu}\right)},
$$

where $G(E)$ denotes the density of states. The density of states $G(E)$ is a measure of how many states exist in a small interval $d E$ around $E$. The probability distribution of the energy $P(E)$ is related to the density of states as

$$
P(E) \propto G(E) \exp \left(-\frac{E}{k_{B} T}\right)
$$




\section{Simulation methods}

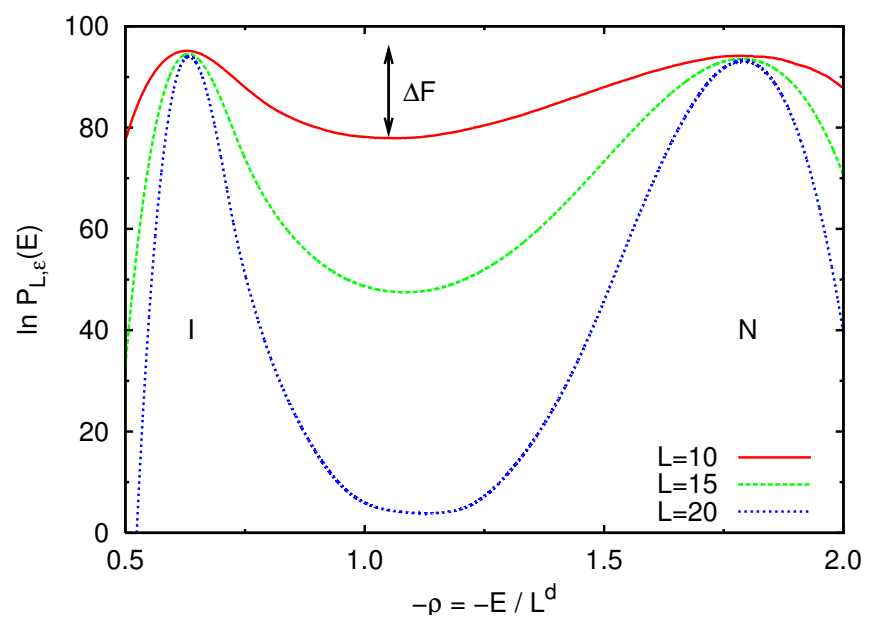

Figure 2.2: Logarithm of the distribution $P(E)$ of the Lebwohl-Lasher model with the exponent $p=10$ on a $d=3$-dimensional lattice, for system sizes $L=10,15,20$ with the inverse temperature $\epsilon$ chosen for each system size to give isotropic (I) and nematic (N) peaks of equal height. The distributions are plotted as functions of negative energy density $-\rho$; the peak on the left thus refers to the isotropic phase, despite having higher energy. The free energy is marked as $\Delta F$ for the $L=10$ system.

If states could be produced in a Monte Carlo simulation with probability according to Eq. (2.11), the simulation would perform a random walk in energy space. That is, all energies would be observed equally often, including those states that contain an interface. This would thus greatly reduce the problem of exponential slowing down.

The problem is that $G(E)$ is a priori unknown. The Wang-Landau algorithm [67, 68] solves this problem by initially setting $G(E)=1$ for all states, and iteratively improves the result such that it converges to the true value. To this end, we choose an energy range $E_{\min }<E_{\mu}<E_{\max }$ in which to perform the simulation; the range is chosen such that both the isotropic and nematic phase are contained. Since the energy in the Lebwohl-Lasher model is continuous, we discretize the energy range into bins or "energy windows", each window being of equal width.

In the first Wang-Landau iteration, we perform generalized spin flips as usual. A move from state $\mu$, with energy $E_{\mu}$, to state $\nu$, with energy $E_{\nu}$, is accepted with probability

$$
P\left(E_{\mu} \rightarrow E_{\nu}\right)=\min \left[1, \frac{G\left(E_{\mu}\right)}{G\left(E_{\nu}\right)}\right] .
$$

Note that this is just the Metropolis algorithm of Eq.2.10 but with the Boltzmann probability replaced by Eq. 2.11). The key difference from "standard" sampling is that each time an energy window $E$ is visited the corresponding estimate of the density of states $G(E)$ is multiplied by some modification factor $f>1$, i.e. $G(E) \rightarrow$ 
$G(E) \cdot f$ (we initially set $f=e \approx 2.72$ ). The effect of this modification is to make it less likely to visit the same state again, and instead visit states that have not yet been visited. Additionally, we keep track of a histogram $H(E)$ counting the number of times each energy window is visited. The random walk is continued until the energy windows have been visited sufficiently often and $H(E)$ is sufficiently flat. 1 This ends the first Wang-Landau single iteration.

For the second iteration, $H(E)$ is reset and $f$ is reduced according to some function that monotonically decreases $f$ to unity; we generally use

$$
f \rightarrow f^{1 / k} .
$$

By using a smaller value of $f$ we make less drastic changes to the density of states. The simulation is then restarted, rebuilding $H(E)$ and updating $g(E)$ as before, until all windows have once again been visited sufficiently often and $H(E)$ is once again sufficiently flat. More iterations then follow, each time using a reduced modification factor $f$, until $f$ is very close to unity (and $H(E)$ hopefully very nearly flat). At this point, the estimate of $G(E)$ has converged to the true density of states, and the energy distribution $P(E)$ can be calculated for any temperature using Eq.(2.12).

To prevent numerical overflow, we calculate $\ln G(E)$ in the simulation: after each move we thus update $\ln [G(E)] \rightarrow \ln [G(E)]+\ln (f)$. Between different Wang-Landau iterations, the "fining" of the modification factor thus follows $\ln f_{\text {new }}=\ln f_{\text {old }} / k$ with some constant $k>1$. Wang-Landau iterations are continued until $\ln f$ is smaller than some cut-off value, whereupon the simulation is complete. The choice of $k$ is important as it affects the accuracy of the measured density of states, as well as the time required for the simulation to complete.

Of note is that early in the simulation, where $f$ is still large and $G(E)$ is being changed considerably, detailed balance is not fulfilled. The distribution being sampled is being dynamically altered, and thus equilibrium will not be obtained. However, toward the end of the Wang-Landau simulation, as the changes to $G(E)$ become negligible, detailed balance is restored.

The Wang-Landau algorithm can be optimized by performing many random walks simultaneously on different or overlapping energy ranges. Performing simulations on smaller energy ranges and combining them after they have finished can save considerable time compared to performing a single simulation over the complete energy range. Most of the results presented in later chapters were indeed obtained by combining data from different Wang-Landau simulations.

\subsubsection{Transmission matrix Wang-Landau algorithm}

Wang-Landau sampling generally works very well; it certainly outperforms the Metropolis algorithm at overcoming free energy barriers and it collects data of high

\footnotetext{
${ }^{1}$ There is a discussion of what is "sufficiently flat" in 68. Generally, we find that choosing a suitable degree of flatness is highly dependent on the system parameters.
} 


\section{Simulation methods}

statistical quality. However, it has been noted that in the limit of long simulation time, the statistical quality of the density of states no longer increases [69, 74]. To solve this problem we use a modified version of the Wang-Landau algorithm based on the transition matrix.

The point to note is that the accept probability in Eq. $(2.13)$ is generally smaller than unity. In fact, a considerable number of moves is rejected thereby wasting valuable computer time. However, it is possible to also use information of rejected moves to construct the density of states. To this end we introduce the quantity

$$
T(\mu \rightarrow \nu)
$$

defined as the number of proposed Monte Carlo moves from a state with energy $E_{\mu}$ to a state with energy $E_{\nu}$, regardless of whether the moves were accepted. The quantity $T$ above is known as a transmission matrix element. The advantage of using the transmission matrix elements is that more information is collected, since now also rejected moves are used, and thus $G(E)$ can be estimated more accurately and quickly than via conventional Wang-Landau sampling.

In terms of the transmission matrix elements, the probability of proposing a move to a state with energy $E_{\nu}$, assuming one is in a state with energy $E_{\mu}$, is given by

$$
\Omega(\mu \rightarrow \nu)=\frac{T(\mu \rightarrow \nu)}{\sum_{\nu} T(\mu \rightarrow \nu)}
$$

These proposal probabilities are related to the density of states by [75]

$$
\frac{G\left(E_{\mu}\right)}{G\left(E_{\nu}\right)}=\frac{\Omega(\nu \rightarrow \mu)}{\Omega(\mu \rightarrow \nu)}
$$

Hence, from the transmission matrix elements the density of states can also be constructed.

Note that if we were to collect information about all proposal statistics, as suggested in Eq.(2.16), we would require a significant amount of computer memory. However, if we perform only single spin flips it is very unlikely that the energy of the system is going to be significantly altered. Starting in a state with energy in "energy window" $i$, an extreme version of this idea would be to count only:

1. how often a move is proposed to a state with energy in the "left" window $i-1$ $\left(H_{\text {left }}\right)$;

2. how often a move is proposed to a state with energy in the "right" window $i+1\left(H_{\text {right }}\right)$;

3. how often a move is proposed to a state in some other window $\left(H_{\text {rest }}\right)$. 
The proposal probabilities are thus given as

$$
T(i \rightarrow i+1)=\frac{H_{\text {right }}}{H_{\text {left }}+H_{\text {right }}+H_{\text {rest }}}
$$

for a proposal to window $i+1$ and

$$
T(i \rightarrow i-1)=\frac{H_{\text {left }}}{H_{\text {left }}+H_{\text {right }}+H_{\text {rest }}}
$$

for a proposal to window $i-1$. The density of states is related to the ratios of $T$ as

$$
\frac{G(i+1)}{G(i)}=\frac{T(i \rightarrow i+1)}{T(i+1 \rightarrow i)} \text {. }
$$

The complete density of states can be calculated recursively as

$$
G(0) \equiv 1, \quad G(1)=\frac{T(0 \rightarrow 1)}{T(1 \rightarrow 0)} G(0)
$$

and so forth for all energy windows. In this implementation the additional memory load consists of only three arrays, which is negligible.

The transmission matrix elements can be used in conjunction with Wang-Landau sampling as follows. In our implementation we distinguish two stages: a "prepare" stage, followed by a "collect" stage. At the start of the prepare stage we set $f=e$ as before and perform Wang-Landau iterations as usual. After each move, accepted or rejected, the density of states $G(E)$, the energy histogram $H(E)$, and the transmission matrix elements are updated. When $H(E)$ has become sufficiently flat the transmission matrix elements are used to construct the density of states, via Eq.2.21), which is then used for the next Wang-Landau iteration. By using the transmission matrix elements the "flatness" criterion can be relaxed tremendously: $G(E)$ can already be calculated accurately via Eq.2.21) when each energy bin has been visited only once. In addition we can reduce the modification factor much more quickly than in conventional Wang-Landau sampling [69]. Typically one can use $k=10$ in Eq.(2.14), as opposed to $k=2$ in the conventional implementation. The modification factor $f$ thus approaches unity extremely rapidly, which marks the end of the "prepare" stage.

We now move onto the "collect" stage. The transmission matrix elements are reset to zero, the histogram $H(E)$ is no longer needed, but for $G(E)$ we retain the estimate obtained in the prepare stage. We now simulate according to the WangLandau acceptance criterion Eq. (2.13), but only the transmission matrix elements are updated after each move, and not $G(E)$ (this corresponds to using $f=1$ ). At this stage, further sampling increases the accuracy of the transmission matrix elements indefinitely [69], and the calculation of $G(E)$ from the transmission matrix elements thus always becomes more accurate. In addition, since during the "collect" stage the density of states used in the acceptance probability of Eq.(2.13) is not altered, detailed balance is strictly obeyed (as opposed to the prepare stage). 
2 Simulation methods

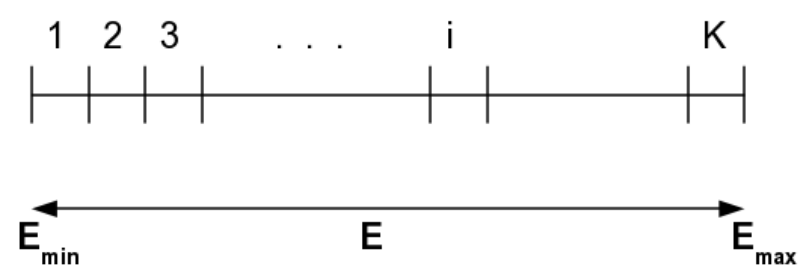

Figure 2.3: Schematic of an energy $E$ range divided into K energy windows of equal width.

\subsubsection{Successive umbrella sampling}

In addition to transition matrix Wang-Landau sampling, we can also use successive umbrella sampling [76] to obtain the density of states $G(E)$. One motivation to do so is to check the consistency of our results, with both methods hopefully yielding the same result for $G(E)$. A second more practical motivation is that by using successive umbrella sampling the amount of CPU time for which the simulation is to run can be specified, ensuring that simulations finish within a certain time limit. This property is especially convenient when quenched disorder is introduced into the system and we require the average of many realizations of disorder (see chapter 6).

Successive umbrella sampling is similar to Wang-Landau sampling, in that we attempt to measure the density of states $G(E)$ of the system. However, the method in which this is done is quite different. With Wang-Landau sampling we perform a random walk over some specified energy interval, sampling each state $\mu$ with a probability inversely proportional to $G\left(E_{\mu}\right)$, and updating $G\left(E_{\mu}\right)$ as we go along. With successive umbrella sampling we perform only one sweep over the energy range, and the density of states is not updated whilst simulating (hence, detailed balance is always obeyed).

We imagine setting the temperature $T \rightarrow \infty$ and perform generalized spin flips accepting each with the Metropolis probability of Eq.2.10). Note that for $T \rightarrow \infty$ Eq. 2.10 implies that all moves are accepted. The obvious question is how can this be a meaningful simulation approach? The trick of successive umbrella sampling is to split the energy range of interest, $E_{\min }<E<E_{\max }$, into "energy windows", as was also done for Wang-Landau sampling. The splitting into windows is shown in Fig. 2.3, where each of the windows is numbered from $i=1, \ldots, K$. Initially we force the simulation to have an energy in either window 1 or window 2 . That is we perform generalized spin flips accepting all, except those which would yield an energy outside of the bounds of windows 1 and 2 (there is rejection after all!). We perform very many moves (typically hundreds of millions) and we count how often the simulation is inside window $1\left(H_{1}\right)$ and in window $2\left(H_{2}\right)$. The ratio of the 
counts is related to the density of states

$$
\frac{G\left(E_{2}\right)}{G\left(E_{1}\right)}=\frac{H_{2}}{H_{1}}
$$

where for $E_{i}$ we take the value of the energy in the center of window $i$. Once the ratio has reached a desired level of statistical accuracy we perform a simulation in windows 2 and 3, from which $G\left(E_{3}\right) / G\left(E_{2}\right)$ can be determined and so forth. By extending this process all the way to the $K$-th window the density of states can be constructed over the desired energy range via recursion (analogous to Eq.(2.21)). This explains the basic successive umbrella sampling algorithm.

An important optimization of successive umbrella sampling is to use the information obtained whilst simulating in windows $(i, i+1)$ for the next (successive) step. To be explicit, assume that the ratio of counts after the first step $F_{1} \equiv H_{2} / H_{1}$ is very large. This means that most time was spend in window 2. For optimal statistics it would be better if windows 1 and 2 were visited equally often. Hence, to better sample windows 2 and 3 we should abandon Eq. 2.10) and instead accept moves according to

$$
A(\mu \rightarrow \nu)= \begin{cases}1 / F_{1} & \text { if } E_{\mu} \in \text { window } 2 \text { and } E_{\nu} \in \text { window } 3 \\ F_{1} & \text { if } E_{\mu} \in \text { window } 3 \text { and } E_{\nu} \in \text { window 2 } \\ 1 & \text { otherwise }\end{cases}
$$

where moves that lead to energies outside of the bounds of windows 2 and 3 are also rejected. We thus assume that if $F_{1}$ is large it is also likely that $F_{2} \equiv H_{3} / H_{2}$ will be large. The above accept probability makes transitions from window $2 \rightarrow 3$ less likely, leading to a measured ratio $F_{2}$ that will be closer to unity. Of course, unless $H_{1} \equiv H_{2}$ either $F_{1}$ or $1 / F_{1}$ will be greater than unity. In this case the appropriate accept probability is simply unity. Note that Eq. (2.22) must be correspondingly modified

$$
\frac{G\left(E_{3}\right)}{G\left(E_{2}\right)}=F_{1} \times \frac{H_{3}}{H_{2}}
$$

and analogously for the subsequent steps.

Similar to Wang-Landau sampling, successive umbrella sampling provides an additional means to obtain the density of states, and thus also the properties of the system at any temperature by virtue of Eq.(2.12). Successive umbrella sampling has the important practical advantage that one can trivially set the length of time one wishes to simulate per window, thereby ensuring that simulations finish within a certain time limit (this does not, of course, ensure that the statistical quality will be sufficient). In this thesis successive umbrella sampling proves to be particularly useful in dealing with systems with quenched disorder (see chapter 6, where results must be averaged over many thousands of realizations of disorder. 
2 Simulation methods 


\section{The Lebwohl-Lasher model}

\section{Contents}

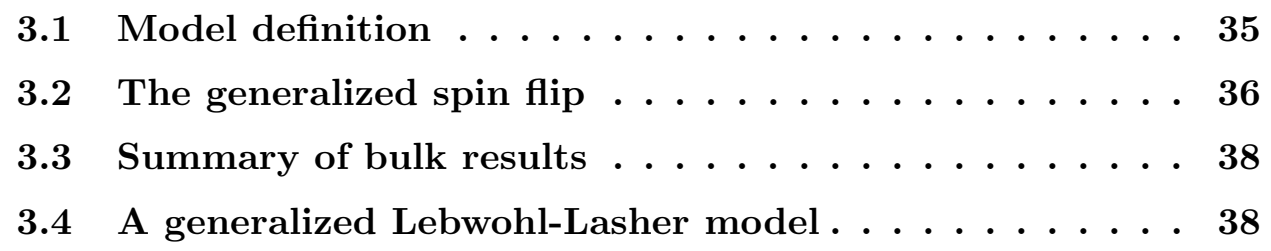

The liquid crystal model that is used as the basis of this thesis is that proposed by Lebwohl and Lasher [64, 77]. The model is defined on a lattice and can be used to describe a thermotropic isotropic-nematic transition. Originally studied in bulk [64, the Lebwohl-Lasher model is easily adapted to tackle the more complicated case of confinement (as we will show in later chapters). The relative simplicity of the Lebwohl-Lasher model allows for very efficient simulations, although some features of real liquid crystals are obviously lost. For example, in real liquid crystals there are density fluctuations; these cannot be reproduced by the Lebwohl-Lasher model. The Lebwohl-Lasher model nevertheless reproduces the isotropic-nematic phase transition remarkably well. In three-dimensional (3D) bulk the model undergoes a weakly first-order isotropic-nematic transition [64, 65] in agreement with both experiments [27] and the theoretical descriptions of chapter 1 .

\subsection{Model definition}

In its original form the Lebwohl-Lasher model is defined on a 3D lattice of form $V=L \times L \times L$, with volume $V$ and side length $L$ and with periodic boundary conditions applied in all directions. A $3 \mathrm{D}$ unit vector $\vec{d}_{i}$ (spin) is placed at each lattice site $i$ and each spin interacts with its nearest neighbors at sites $j$ via the Hamiltonian

$$
\mathcal{H}=-\epsilon \sum_{\langle i j\rangle}\left|\vec{d}_{i} \cdot \vec{d}_{j}\right|^{p}
$$

with the exponent $p=2$ and coupling constant $\epsilon$. The term $\langle i j\rangle$ denotes the sum over all nearest neighbors and a factor $1 / k_{B} T$ is incorporated into the coupling constant, with $k_{B}$ the Boltzmann constant and $T$ the temperature. In this work $\epsilon>0$, and it thus plays the role of inverse temperature. The use of the absolute value ensures that the system remains symmetric under inversion $\vec{d}_{i} \rightarrow-\vec{d}_{i}$, as is 


\section{The Lebwohl-Lasher model}

required for liquid crystals (see section 1.2.1). Note that for $p=2$ the Hamiltonian of Eq.(3.1) can also be expressed in terms of the second Legendre polynomial, as was done in the original paper 64]. For this reason, transition temperatures quoted in this thesis differ by a factor of $3 / 2$ when compared to many earlier works [64, 65, 78].

\subsection{The generalized spin flip}

When performing simulations using the Lebwohl-Lasher model, we use the generalized spin flip Monte Carlo move, as was already mentioned in section 2.2.4. In the Ising model, with spins $s_{i}= \pm 1$, a spin flip consists of changing the direction (sign) of the spin. For the Lebwohl-Lasher model this move would be pointless, as the system is invariant under such operations. Furthermore, such a move would not be ergodic for the Lebwohl-Lasher model as the spins are continuous vectors. In the generalized spin flip, one therefore randomly selects one of the spins $\vec{d}_{i}$ and assigns it a completely new and randomly selected orientation. As the spins are 3D the random selection of the orientation implies the selection of a random point on the surface of a sphere. To this end we use a simple rejection-type approach [79] which proceeds as follows:

1. generate three random numbers $x, y, z$ in the range $[-1,1]$;

2. calculate $r=x^{2}+y^{2}+z^{2}$;

3. if $r>1$ return to step 1 , otherwise proceed to step 4 ;

4. normalize $x, y, z$ such that $r=1$ and accept the orientation.

Using generalized spin flips we can now clearly see that the proposition probabilities cancel from the Metropolis accept probability (see Eq. (2.9)). The selection probabilities of such moves $g(\mu \rightarrow \nu)$ between states $\mu$ and $\nu$ and the reverse move $g(\nu \rightarrow \mu)$ are symmetric

$$
g(\mu \rightarrow \nu)=g(\nu \rightarrow \mu)=\frac{1}{4 \pi} \frac{1}{N},
$$

where the last term reflects the combined probability of selection one of the spins and the orientation. Now applying the condition of detailed balance one sees that the proposition probabilities cancel, and so generalized spin flips can be accepted using the Metropolis choice

$$
P_{\text {acc }}(\mu \rightarrow \nu)=\min \left[1, e^{-\beta\left(E_{\nu}-E_{\mu}\right)}\right]
$$

where $\beta$ is the inverse temperature, and $E_{\mu}$ and $E_{\nu}$ are the energies of the system in the states $\mu$ and $\nu$ respectively. 


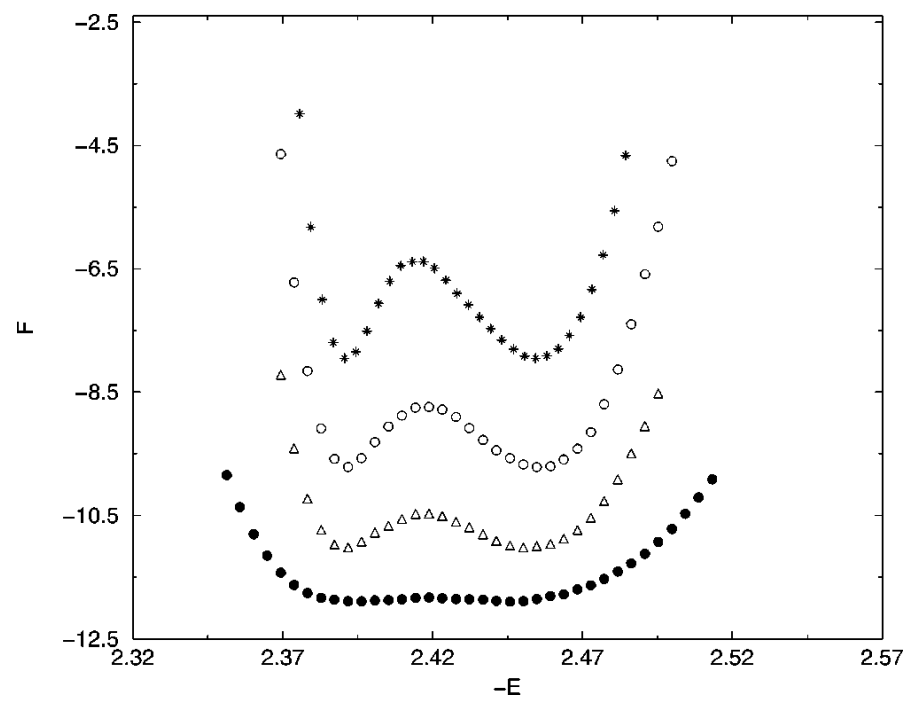

Figure 3.1: Free energy $F(E)=-k_{B} T \ln P(E)$ as a function of energy density $E$ of the Lebwohl-Lasher model with $p=2$. Data is shown for system sizes $L=30,50,60,70$ (in order of rising data sets). The free energy barrier $\Delta F$ of the transition is calculated for each system size as the difference in free energy between the two minima and the maximum in-between. Note that $\Delta F$ is very small: even for the $L=70$ system $\Delta F \approx 2 k_{B} T$. Reprinted figure with permission from Ref. [80]. 
3 The Lebwohl-Lasher model

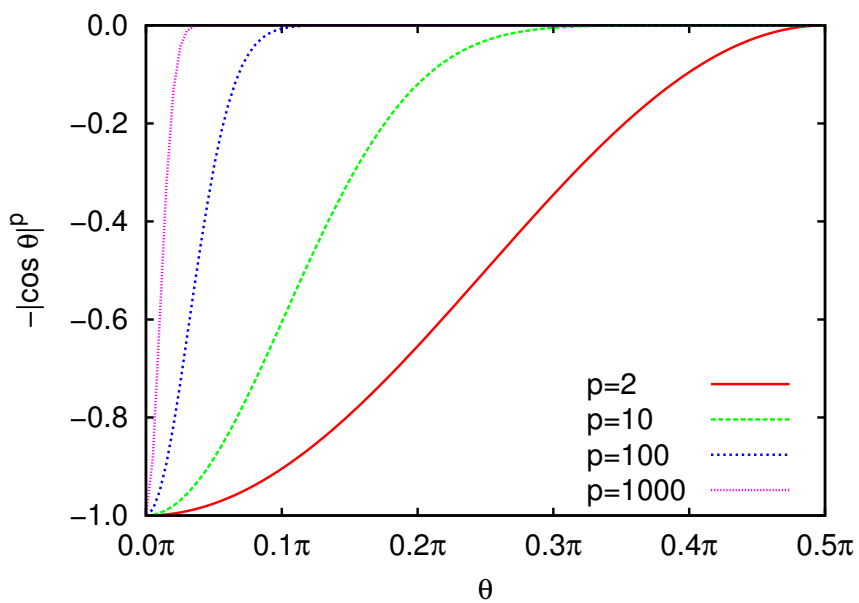

Figure 3.2: The pair interaction of Eq. (3.1) as a function of the angle $\theta$ between two spins $i$ and $j$ for various values of the exponent $p$, where $\cos \theta=\vec{d}_{i} \cdot \vec{d}_{j}$. As $p \rightarrow \infty$ the interaction increasingly resembles a $\delta$-function.

\subsection{Summary of bulk results}

The Lebwohl-Lasher model in its original form - with spins interacting via Eq.(3.1) using $p=2$ on a 3D lattice with periodic boundary conditions - undergoes a firstorder isotropic-nematic phase transition. However, the transition is very weak and can only be shown convincingly in simulations using very large lattices, as shown in Fig. 3.1. 11 The double-peaked structure of the energy probability distribution $P(E)$ reflecting the phase coexistence (typical of first-order phase transitions) only appears in very large systems. Even for the largest system shown, with $L=70$, the free energy barrier $\Delta F$ of interface formation is only $\Delta F \approx 2 k_{B} T$.

\subsection{A generalized Lebwohl-Lasher model}

A simple modification of Eq.(3.1), which plays a major role in the results to come, is to change the value of the exponent $p$. If one pictures a "rod-type" molecule, the effect of making $p$ large can be thought of as stretching the molecule so that it is longer and thinner. For large $p$ the pair-interaction becomes sharper, as shown in Fig. 3.2. The effect is that for large $p$ neighboring spins can appreciably lower the energy by aligning, but once they are unaligned the effect on the energy is small. This somewhat resembles the interaction in the Potts model [81]. In fact, as $p \rightarrow \infty$ the pair interaction of Eq. 3.1) approaches a $\delta$-function.

\footnotetext{
${ }^{1}$ The Hamilton used in Fig. 3.1 equals $\mathcal{H}=-\epsilon \sum_{\langle i j\rangle}\left\{\frac{3}{2}\left(\vec{d}_{i} \cdot \vec{d}_{j}\right)^{2}-\frac{1}{2}\right\}$ giving a different prefactor from our Eq. 3.1).
} 

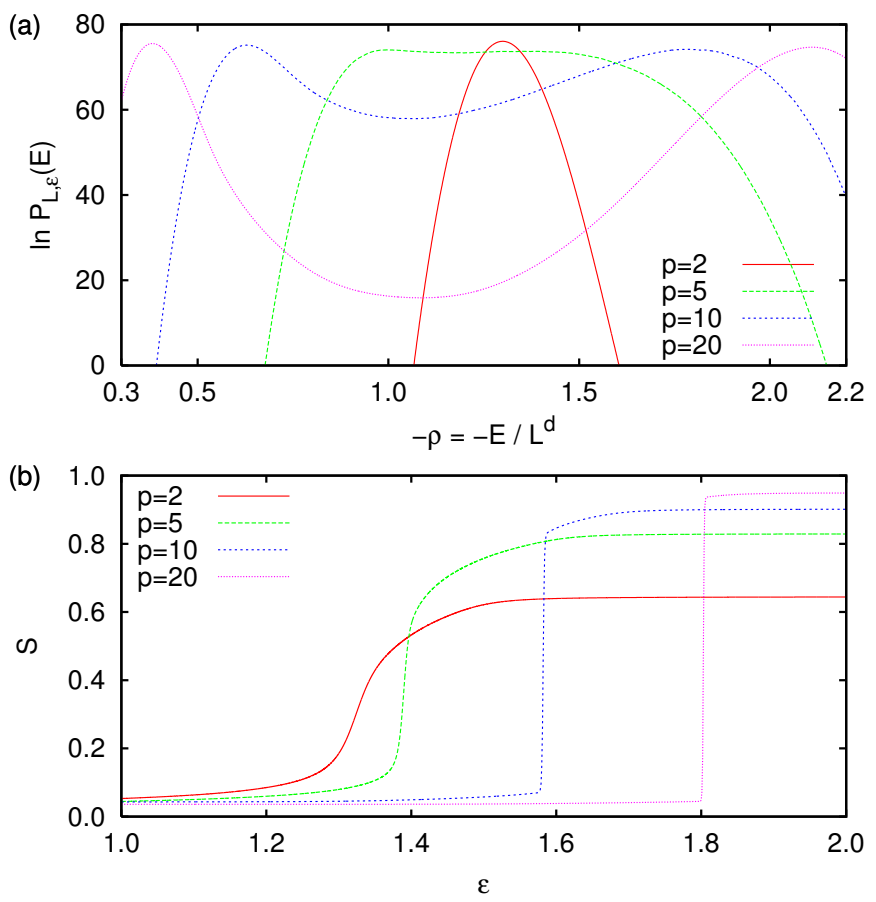

Figure 3.3: The effect of the exponent $p$ in Eq. 3.1 on the isotropic-nematic transition in three dimensions. (a) Logarithm of the energy probability distribution $P(E)$ obtained in simulations of Eq.(3.1) using $p=2,5,10,20$, all with $L=10$. The distributions are plotted as functions of negative energy density $-\rho$. Hence, the left peaks reflect the isotropic phase and the right peaks the nematic phase. The inverse temperature $\epsilon$ has been chosen for each distribution individually to give peaks of equal height, permitting an easy calculation for the free energy barrier $\Delta F$ (to be discussed in chapter 4). (b) Plots of the corresponding nematic order parameters $S$ versus inverse temperature $\epsilon$ for the same systems. The characteristic "jump" of the first-order phase transition becomes stronger as $p$ increases. 


\section{The Lebwohl-Lasher model}

It has been noted [82, 83, 84, 85] and mathematically proven [63, 86, 87] that for such interactions the first-order transition becomes stronger. We show this explicitly in Fig. 3.3. (a) where the logarithm of the probability distribution $P(E)$ of the energy is plotted against negative energy density for different values of $p$ for fixed $L=10$. We observe a free energy barrier (indicating a first-order phase transition) which increases profoundly with $p$. In (b) we show the nematic order parameter $S$ versus "inverse temperature" $\epsilon$. The characteristic "jump" of the order parameter also becomes more pronounced with increasing $p$.

\section{Outlook}

The Lebwohl-Lasher model can also be easily modified in other ways, for example by applying walls or adding quenched disorder. Variations of the Lebwohl-Lasher model along these lines are used throughout this thesis. The interesting finding is that, depending on the value of $p$, qualitatively different scenarios for the isotropicnematic transition are found. This shows that phase transitions in Eq.(3.1) are not governed by any strict universality class. 


\title{
4 Finite-size scaling of the isotropic-nematic phase transition
}

\author{
Contents \\ 4.1 Introduction $\ldots \ldots \ldots \ldots \ldots \ldots \ldots \ldots$ \\ $4.2 \quad$ Finite-size scaling $\ldots \ldots \ldots \ldots$. . . . . . . . . . 42 \\ 4.3 Simulations using Lebwohl-Lasher type models . . . . . 43 \\ 4.3 .1 Model and simulation method . . . . . . . . . . . . . . . 44 \\ 4.4 Results and analysis . . . . . . . . . . . . . 46 \\ 4.4 .1 Determining the order of the transition . . . . . . . . 46 \\ 4.4 .2 Extrapolation of $\epsilon_{L, c}$ : Potts $1 / L^{d}$ scaling. . . . . . . . . 49 \\ 4.4.3 Extrapolation of $\epsilon_{L, k}:$ application of the Borgs-Kotecký

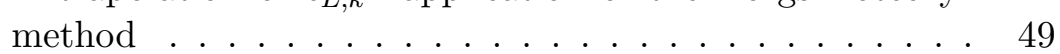 \\ 4.4 .4 Latent heat density . . . . . . . . . . . . . . 55 \\ 4.5 Summary $\ldots \ldots \ldots \ldots \ldots \ldots$
}

\subsection{Introduction}

First-order phase transitions are characterized by discontinuities in the first derivatives of the free energy. In thermotropic systems, at a certain temperature the order parameter will jump suddenly between the disordered and ordered phases. As a result observables, such as the susceptibility and the specific heat, have $\delta$-function singularities precisely at this temperature. These effects are due to phase coexistence [88].

In contrast continuous phase transitions do not have discontinuities in the first derivative of the free energy, and do not show phase coexistence. As the transition is approached the correlation length $\xi$ diverges to infinity and the behavior of, for example, the susceptibility or the specific heat is characterized by critical exponents, so-called because the region near the transition is known as the critical region [89].

A first-order phase transition with its characteristic discontinuities, however, has no critical region (and hence no critical exponents). By virtue of the transition being discontinuous, the divergences typical of continuous transitions do not occur. 


\section{Finite-size scaling of the isotropic-nematic transition}

Instead of becoming infinite $\xi$ remains finite, even precisely at the transition (except for systems with broken continuous symmetry [90]). For this reason both ordered and disordered regions coexist precisely at the transition.

In this chapter we analyse finite-size effects at the first-order isotropic-nematic transition by computer simulation. In particular, we investigate the link between scaling relations derived for the Potts model and their applicability to the LebwohlLasher model.

\subsection{Finite-size scaling}

The divergences which occur at phase transitions in the thermodynamic limit do not occur in finite-sized systems. Instead one typically observes rounding effects, where peak heights of thermodynamic observables, such as the susceptibility or the specific heat increase with system size, and the locations of these maxima are shifted, as seen in Fig. 4.1. Although such effects occur for both first-order and continuous phase transitions, their behavior is dependent on the type of transition. Knowledge of these finite-size effects is particularly useful when performing simulations. Instead of simulating as large a system as possible and hoping that it approximates the thermodynamic limit, one can extrapolate from small systems, saving time and obtaining more accurate results. This method of extrapolation is called finite-size scaling . In order to perform finite-size scaling one needs to have knowledge of how the system will scale. Scaling behavior is different for systems undergoing either continuous or first-order phase transitions.

The effects of rounding at a continuous transition are due to $\xi$ being limited by the side length $L$ of the system. Approaching the transition $\xi \rightarrow \infty$, and therefore at some stage $\xi$ is going to be limited by $L$, because the simulation box is "too small" to capture the relevant length scale [89].

The scaling effects at first-order phase transitions are fundamentally different because $\xi$ does not diverge to infinity as the transition is approached. At the transition ordered and disordered phases coexist, and finite-size effects are thus governed by the volume $V=L^{d}$ of the system, with $d$ the spatial dimension [91]. Hence, nontrivial critical exponents do not occur. Since two phases coexist, the order parameter features two peaks, i.e. it is bimodal. As $L \rightarrow \infty$ and the thermodynamic limit is approached the bimodal distribution becomes more and more extreme until it becomes just two $\delta$-peaks situated at values of the order parameter $S_{1}$ and $S_{2}$ and is thus given by 92

$$
P_{\infty}(S)=\frac{\delta\left(S-S_{1}\right)+\delta\left(S-S_{2}\right)}{2} .
$$

To be explicit: for the isotropic-nematic transition, where the coexistence is between the isotropic and nematic phase, we would have $S_{1}=0$ and $S_{2}=S_{\text {nem }}$, respectively, with $S$ the nematic order parameter defined in section 1.4.1. The susceptibility in $d$ dimensions $\chi=L^{d}\left(\left\langle S^{2}\right\rangle-\langle S\rangle^{2}\right)$ is therefore trivially $\propto L^{d}$. As the system volume 


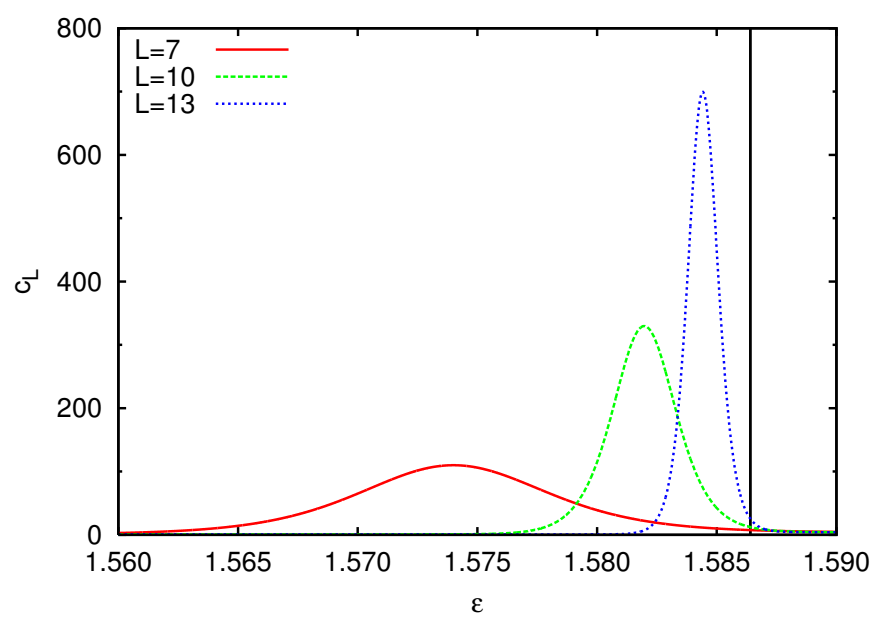

Figure 4.1: Specific heat $c_{L}=V\left(\left\langle E^{2}\right\rangle-\langle E\rangle^{2}\right) / V$ against inverse temperature $\epsilon$ for the three-dimensional (3D) Lebwohl-Lasher model with $p=10$ (see Eq.(4.4) ) using system side lengths $L=7,10,13$. The peaks grow in height and narrow in width with increasing system size. The peaks shift towards the thermodynamic limit value, shown by the vertical line at $\epsilon_{\infty}=1.5864$.

increases, the squared width of $\chi$ narrows $\propto L^{-d}$, owing to the central limit theorem 93. obtaining the $\delta$-function limit of Eq. 4.1) for an infinitely large system [94, 95].

It is not just the height and widths of the maxima that change with system size, but their positions are also shifted from their thermodynamic limit locations; these shifts also have a $1 / L^{d}$ dependence [96]. Using finite-size scaling laws we would therefore expect to locate first-order phase transitions from finite systems with shifts vanishing with $1 / L^{d}$.

\subsection{Simulations using Lebwohl-Lasher type models}

In this chapter we use the (continuous spin) Lebwohl-Lasher model [64, 77] to analyse the first-order isotropic-nematic phase transition; the Lebwohl-Lasher model was described in detail in chapter 3 . However, the vast majority of finite-size scaling results for first-order transitions were derived for the Potts model [97]. The Potts model is fundamentally different to the Lebwohl-Lasher model as it has discrete spins, i.e. the spins at the lattice sites are only allowed to assume $q$ possible values. This has some interesting consequences when the corresponding scaling relations are applied to the Lebwohl-Lasher model. For example, in the Potts model, the specific heat peak shifts from its thermodynamic limit value in finite systems as

$$
\epsilon_{L, c}=\epsilon_{\infty}-A_{\text {Potts }} / L^{d}+\mathcal{O}\left(1 / L^{2 d}\right),
$$




\section{Finite-size scaling of the isotropic-nematic transition}

with proportionality constant $A_{\text {Potts }}$, the thermodynamic limit transition inverse temperature $\epsilon_{\infty}$, and $\epsilon_{L, c}$ the inverse temperature where the specific heat maximum exists in a finite system of side length $L[96,98]$. A $A_{\text {Potts }}$ is then related to the latent heat density $\lambda_{L}$ and the number of Potts states $q$ as

$$
A_{\text {Potts }}=\ln q / \lambda_{\infty}
$$

Because the Lebwohl-Lasher model has continuous spins, the significance of $A_{\text {Potts }}$ appears to be lost, although a numerical value for it could still be obtained by fitting to finite-size simulation data. This would enable us to assign an effective discrete number of states to the Lebwohl-Lasher model, even though the model is continuous.

The above subtlety appears to have gone unnoticed until now. Despite the scaling equation Eq. (4.2) being derived for the Potts model, it has been applied to the Lebwohl-Lasher model without question, and shown to work remarkably well for this model also [99, 80]. With this observation in mind, it could be hoped that other Potts model scaling relations also have significance for the Lebwohl-Lasher model. One such case is a method of Borgs and Kotecký for calculating the thermodynamic limit inverse temperature $\epsilon_{\infty}$ [98, 100, 101]. The appealing property of the latter method is that the finite-size effects decay exponentially, i.e. much faster than the power-law decay of Eq. 4.2), making it possible for $\epsilon_{\infty}$ to be obtained using smaller systems and thus saving valuable computation time. In this chapter we investigate if this approach indeed works.

\subsubsection{Model and simulation method}

The original Lebwohl-Lasher model (described in chapter 3 ) is defined on a threedimensional (3D) periodic lattice of form $V=L \times L \times L$, with volume $V$ and system side length $L$ with periodic boundary conditions. A 3D unit vector $\vec{d}_{i}$ is placed on each lattice site $i$ and these interact with their nearest neighbors at sites $j$ via

$$
\mathcal{H}=-\epsilon \sum_{\langle i j\rangle}\left|\vec{d}_{i} \cdot \vec{d}_{j}\right|^{p}
$$

with exponent $p=2$ and coupling constant $\epsilon$. The $\langle i j\rangle$ denotes the sum over nearest neighbors. The factor $1 / k_{B} T$ is incorporated into the coupling constant, with $k_{B}$ the Boltzmann constant and $T$ the temperature, so that $\epsilon>0$ is playing the role of the inverse temperature. In this form the model undergoes a first-order phase transition, as has been previously discussed [77, 102, 80, 65, 99] at $\epsilon \approx 1.34$ [103].

As described in section 3.3 , to observe the first-order isotropic-nematic transition of the "original" Lebwohl-Lasher model (i.e. with $p=2$ ) one needs very large system sizes $(L \geq 70$ in 3D [80]). For this reason, in this chapter we use the modification of section 3.4, increasing the value of the exponent $p$ in Eq. (4.4) and thus making the phase transition more strongly first-order. For example, with $p=10$ in three dimensions, phase coexistence can be easily observed with systems as small as $L=10$, 


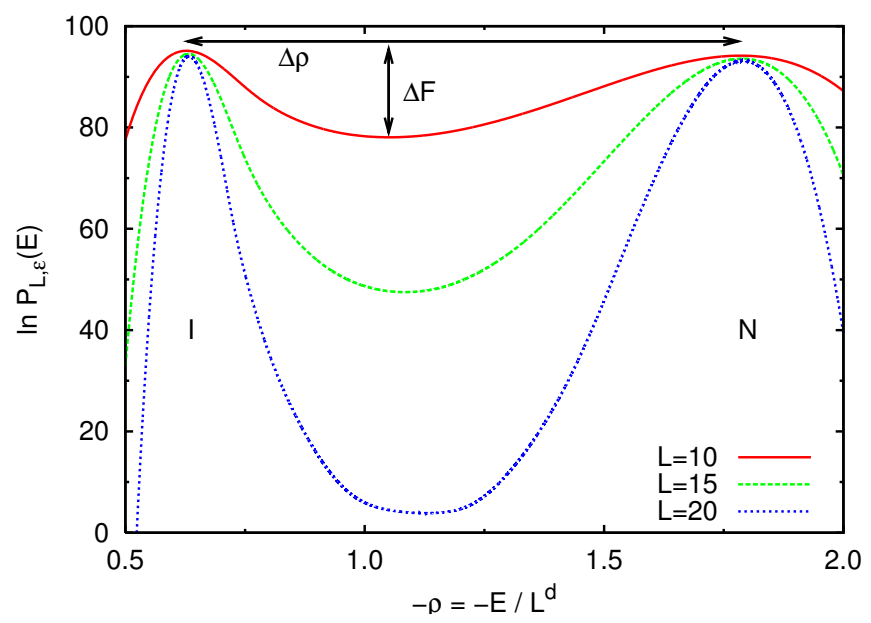

Figure 4.2: Logarithm of the energy density probability distribution $P_{L, \epsilon}(E)$ of Eq. (4.4) with $p=10$ in $3 \mathrm{D}$ for several system sizes $L$. The distribution is distinctly bimodal, characteristic of a first-order transition. The distributions are plotted as functions of $-\rho=E / V$. Therefore, the peak on the left labeled $I$ refers to the isotropic phase, whereas the peak on the right labeled $N$ refers to the nematic phase. The inverse temperature $\epsilon$ has been chosen to give peaks of equal height, permitting an easy calculation for the free energy barrier $\Delta F$ between the two phases. The latent heat density $\Delta \rho$ and $\Delta F$ are marked for the $L=10$ system by the horizontal and vertical arrows, respectively. 
as shown in Fig. 4.2. Hence, to accurately study finite-size effects at the first-order isotropic-nematic transition we proceed with this modified version of the LebwohlLasher model. We perform simulations not only on 3D systems, but also on 2D systems, which we model as a single layer of spins, i.e. with volume $V=1 \times L \times L$, but still keeping the spin vectors $\vec{d}_{i}$ three-dimensional.

Similar to previous simulations of the Lebwohl-Lasher model [99, 80], our simulations are based on the order parameter distribution. Using the energy $E$ as order parameter, we use transmission matrix Wang-Landau sampling [69], as described in section 2.4.1 to measure $P_{L, \epsilon}(E)$ as accurately as possible, where $P_{L, \epsilon}(E)$ is the probability of observing energy $E$ in a system of side length $L$ at inverse temperature $\epsilon$. To "speed-up" our simulations, we often split the energy range of interest into many smaller energy intervals, with a single processor working on each interval. Once all individual simulations have been completed we combine the results from all energy intervals together to reproduce the full distribution. Because we simulate systems as large as $L=25$ in $3 \mathrm{D}$ and $L=100$ in 2D, sub-division of the energy range turned out to be crucial. For 3D systems of side length $L=10$ it is found that a single simulation lasting approximately one day on a $2.66 \mathrm{GHz}$ processor suffices. However, for $L=15$ (already more than $3 \times$ the number of spins) it is more efficient to split the energy range into $5-10$ intervals.

\subsection{Results and analysis}

Because we are interested in checking how first-order scaling laws of the Potts model apply to the Lebwohl-Lasher model, we perform simulations of Eq. (4.4) which give strongly first-order phase transitions. This is achieved in $3 \mathrm{D}$ and $2 \mathrm{D}$ by considering:

1. 3D lattices with $p=5-45$;

2. 2D lattices with $p=20-50$.

The general behavior of the model is well-known for the first scenario. The original Lebwohl-Lasher model, i.e. with $p=2$, already undergoes a first-order phase transition. As has been shown in section 3.4, increasing $p$ will make the transition stronger. It is however less well-known that a Lebwohl-Lasher type model can also undergo a first-order phase transition on a $2 \mathrm{D}$ lattice. This happens when $p$ is sufficiently large [82, 63, 87].

\subsubsection{Determining the order of the transition}

In the vicinity of a first-order phase transition the order parameter distribution becomes bimodal, as shown in Fig. 4.2. The free energy barrier $\Delta F$ of interface formation (see section 2.3) is given by the logarithm of the height difference between the peaks of the distribution and the minimum in-between (vertical arrow 
4.4 Results and analysis

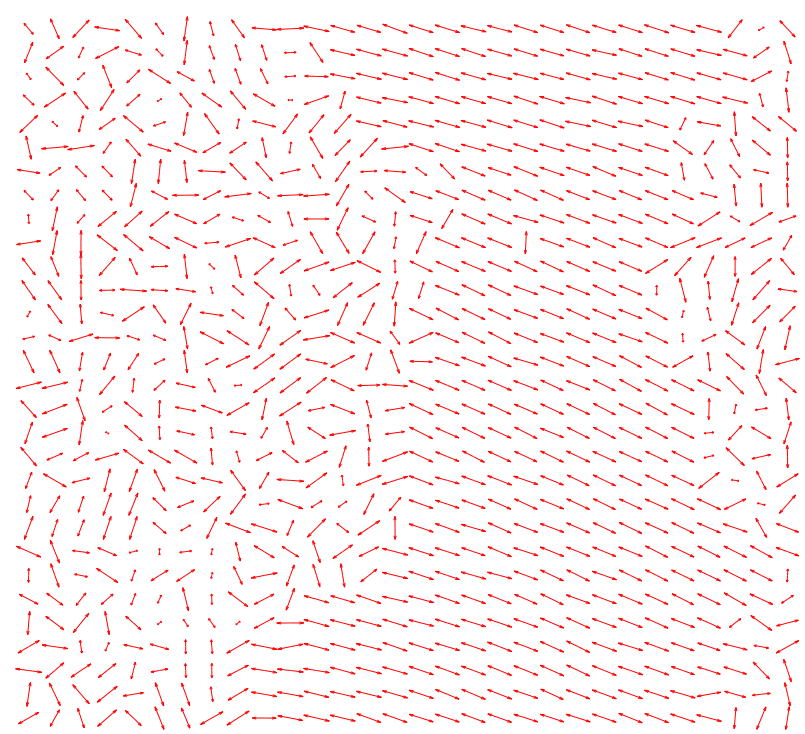

Figure 4.3: Simulation snapshot of the Lebwohl-Lasher model with $p=20$ in 2D with system side length $L=30$ at phase coexistence. Clearly visible are the two interfaces, each of length $L$. 


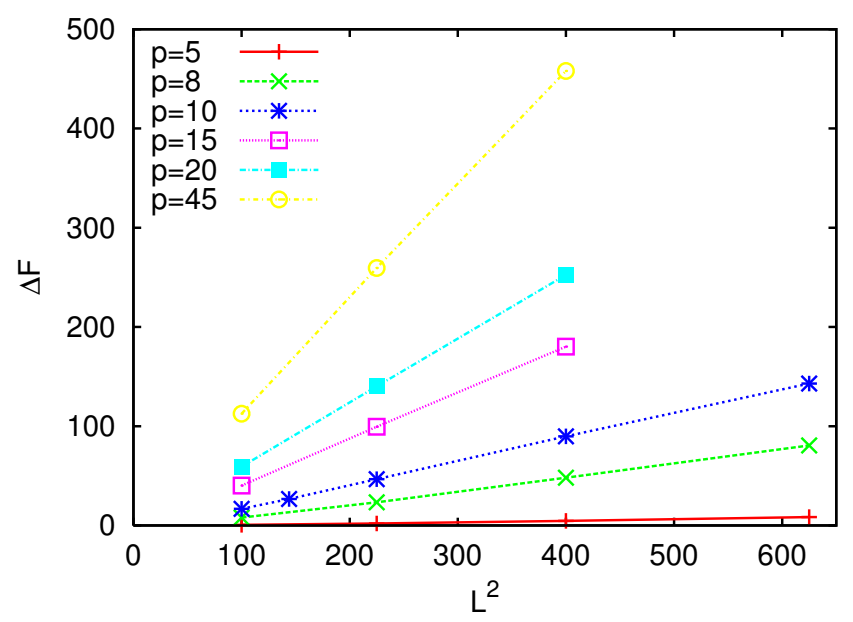

Figure 4.4: Verification of the first-order nature of the isotropic-nematic transition using finite-size scaling of the free energy barrier $\Delta F$ in $d=3$ dimensions. For the chosen values of $p$ we observe a linear increase of $\Delta F$ with $L^{d-1}=L^{2}$, from which we conclude the transitions are first-order.

in Fig. 4.2). At a first-order phase transition, $\Delta F$ corresponds to the free energy required for the formation of an interface between ordered (nematic) and disordered (isotropic) domains [71]. It is expected that the $\Delta F$ barrier grows linearly with the area, i.e. $\Delta F \propto L^{d-1}$. This can easily be inferred from a simulation snapshot taken at the minimum of $P_{L, \epsilon}(E)$. For a $2 \mathrm{D}$ lattice with periodic boundaries, the interface between the domains would stretch from one side of the box to the other, as seen in Fig. 4.3. The free energy required for its formation is proportional to the box side length

$$
\Delta F=2 \sigma L \quad(D=2),
$$

with $\sigma$ the line tension, and where the factor-of-two accounts for the fact that two interfaces are present due to the periodic boundaries. Similarly, for a 3D box this line-interface has to be replaced by a surface-interface, and it therefore yields

$$
\Delta F=2 \sigma L^{2} \quad(D=3) .
$$

By performing simulations for various $L$ and checking whether $\Delta F \propto L^{d-1}$ is observed thus provides a good test for detecting whether a system undergoes a first-order phase transition in the thermodynamic limit $L \rightarrow \infty$ [104, 105]. At a continuous phase transition $\Delta F$ is independent of $L$, whereas $\Delta F$ will vanish altogether if no transition at all exists in the thermodynamic limit.

As can be seen in Fig. 4.4, 3D lattices with $p=5-45$ show a linear increase of $\Delta F$ with $L^{2}$. In Fig. 4.5 we see linear increases of $\Delta F$ with $L$ for $2 \mathrm{D}$ lattices with $p=20-50$. Therefore, we can continue with further study safe in the knowledge that our systems with the chosen $p$ undergo first-order isotropic-nematic transitions. 


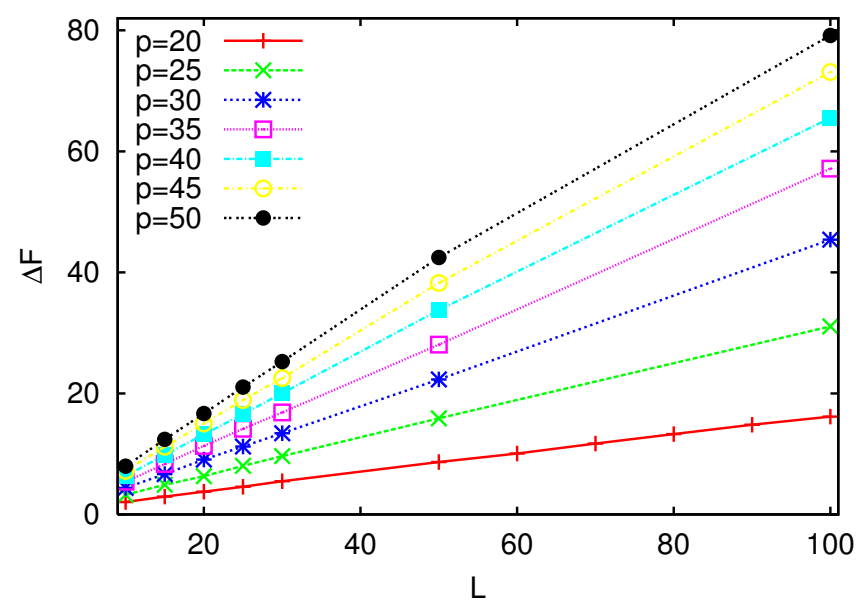

Figure 4.5: The analog of Fig. 4.4 but for $d=2$ dimensional lattices with $p=20-50$. In this case a first-order transition yields $\Delta F \propto L$.

\subsubsection{Extrapolation of $\epsilon_{L, c}$ : Potts $1 / L^{d}$ scaling}

Now that we know a first-order transition exists for the chosen $p$, we can attempt to find the inverse temperature $\epsilon_{\infty}$ at which the phase transition takes place. For several values of the system size $L$, we calculate the specific heat as function of the inverse temperature $\epsilon$, where the specific heat is given by

$$
c_{L}=\frac{\left\langle E^{2}\right\rangle-\langle E\rangle^{2}}{L^{d}},
$$

and $E$ the energy given by Eq. (4.4). We then locate the inverse temperature $\epsilon_{L, c}$ where $c_{L}$ reaches its maximum, see also Fig. 4.1, and extrapolate the latter using the Potts finite-size scaling relation of Eq.(4.2). Example fits are shown in Fig. 4.6 where $\epsilon_{L, c}$ converges to $\epsilon_{\infty}$ as $1 / L^{d}$ for both $p=10$ in $3 \mathrm{D}$ and $p=20$ in $2 \mathrm{D}$. The fit parameters $\epsilon_{\infty}$ and $A_{\text {Potts }}$ are given in Table 4.1 for $3 \mathrm{D}$ systems and in Table 4.2 for $2 \mathrm{D}$ systems. As it has already been shown [99, 80] that the scaling relation Eq. (4.2) works well with the "original" Lebwohl-Lasher model, it is no surprise that our data are well-described by this relation also.

\subsubsection{Extrapolation of $\epsilon_{L, k}$ : application of the Borgs-Kotecký method}

We now use a different finite-size scaling method to locate the isotropic-nematic transition namely the Borgs-Kotecký method. As we had briefly stated before, this 
4 Finite-size scaling of the isotropic-nematic transition

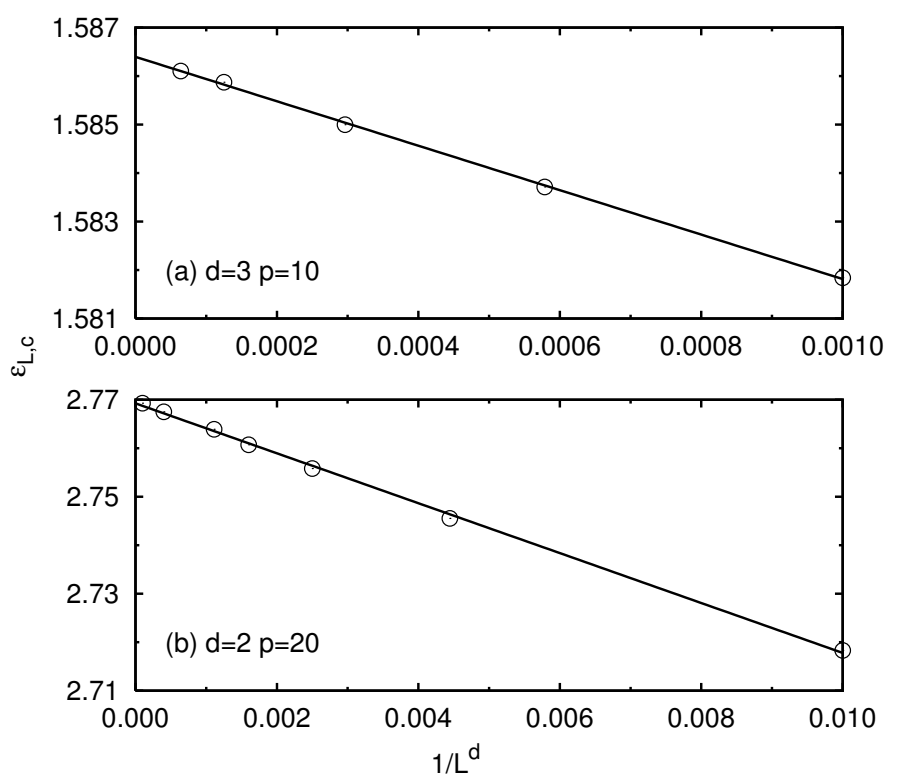

Figure 4.6: Estimation of $\epsilon_{\infty}$ via the extrapolation of $\epsilon_{L, c}$ of the specific heat maximum using Eq.(4.2). (a) Data for $p=10$ in 3D, where $\epsilon_{\infty}=1.5864$. (b) Data for $p=20$ in 2D, where $\epsilon_{\infty}=2.7695$. Additional results for different $p$, as well as the values of the fit parameters, are summarized in Table 4.1 and Table 4.2 .

Table 4.1: Properties of the isotropic-nematic transition of Eq.4.4) for various $p$ on 3D lattices. Listed are fit parameters $\epsilon_{\infty, c}$ and $A_{\text {Potts }}$ of Eq.4.2), the best estimate $\epsilon_{\infty, k}$ obtained from the convergence of $\epsilon_{L, k}$ along $k_{\mathrm{opt}}$, the logarithm of $k_{\mathrm{opt}}$ with uncertainty $\Delta k$, the latent heat density $\lambda_{\infty}$, and the ratio $\ln k_{\text {opt }} / \lambda_{\infty}$.

\begin{tabular}{c|cc|cc|cc}
$p$ & $\epsilon_{\infty, c}$ & $A$ & $\epsilon_{\infty, k}$ & $\ln k_{\mathrm{opt}} \pm \Delta k$ & $\lambda_{\infty}$ & $\ln k_{\mathrm{opt}} / \lambda_{\infty}$ \\
\hline 5 & 1.3969 & 6.62 & $1.3970 \pm 0.001$ & $2.7 \pm 0.6$ & $0.358 \pm 0.010$ & $5.9-9.2$ \\
8 & 1.5207 & 5.28 & $1.5207 \pm 0.001$ & $5.0 \pm 0.2$ & $0.908 \pm 0.005$ & $5.3-5.7$ \\
10 & 1.5864 & 4.28 & $1.5864 \pm 0.001$ & $5.3 \pm 0.5$ & $1.156 \pm 0.005$ & $4.2-5.0$ \\
15 & 1.7126 & 3.94 & $1.7126 \pm 0.001$ & $6.0 \pm 0.2$ & $1.523 \pm 0.002$ & $3.8-4.1$ \\
20 & 1.8063 & 3.76 & $1.8063 \pm 0.001$ & $6.4 \pm 0.3$ & $1.727 \pm 0.002$ & $3.5-3.9$ \\
45 & 2.0838 & 3.55 & $2.0838 \pm 0.001$ & $8.1 \pm 1.0$ & $2.120 \pm 0.001$ & $3.3-4.3$
\end{tabular}


Table 4.2: Similar to Table 4.1 but for 2D lattices.

\begin{tabular}{c|cc|cc|cc}
$p$ & $\epsilon_{\infty, c}$ & $A$ & $\epsilon_{\infty, k}$ & $\ln k_{\mathrm{opt}} \pm \Delta k$ & $\lambda_{\infty}$ & $\ln k_{\mathrm{opt}} / \lambda_{\infty}$ \\
\hline 20 & 2.7695 & 5.18 & $2.7698 \pm 0.001$ & $4.1 \pm 0.4$ & $0.7145 \pm 0.001$ & $5.2-6.3$ \\
25 & 2.8678 & 4.84 & $2.8679 \pm 0.001$ & $4.5 \pm 0.2$ & $0.8900 \pm 0.001$ & $4.8-5.3$ \\
30 & 2.9517 & 4.69 & $2.9517 \pm 0.001$ & $4.8 \pm 0.2$ & $1.0023 \pm 0.001$ & $4.6-5.0$ \\
35 & 3.0240 & 4.58 & $3.0241 \pm 0.001$ & $5.2 \pm 0.6$ & $1.0832 \pm 0.001$ & $4.2-5.4$ \\
40 & 3.0882 & 4.52 & $3.0882 \pm 0.001$ & $5.2 \pm 0.2$ & $1.1432 \pm 0.001$ & $4.4-4.7$ \\
45 & 3.1456 & 4.47 & $3.1455 \pm 0.001$ & $5.2 \pm 0.5$ & $1.1936 \pm 0.001$ & $3.9-4.8$ \\
50 & 3.1976 & 4.50 & $3.1976 \pm 0.001$ & $5.6 \pm 0.5$ & $1.2320 \pm 0.001$ & $4.1-5.0$
\end{tabular}

method was also derived for the Potts model, and shown to yield finite-size effects that vanish exponentially in $L$, which should prove to be more efficient. To apply this method, we define the number $k$ as the ratio of the areas under the isotropic $W_{I}$ and nematic peaks $W_{N}$ of $P_{L, \epsilon}(E)$

$$
W_{N} / W_{I}=k,
$$

which will typically depend on the inverse temperature $\epsilon$, as well as on the system size $L$. We also introduce the inverse temperature $\epsilon_{L, k}$, defined as that inverse temperature where Eq. 4.8) is obeyed. The key idea is that, in the limit $L \rightarrow \infty$, there is only one inverse temperature $\epsilon_{\infty}$ where $P_{L, \epsilon}(E)$ is bimodal: at a slightly lower inverse temperature the system is fully isotropic; at a slightly higher inverse temperature the system is fully nematic. Hence, a non-trivial solution of Eq.(4.8) (by which we mean $k \neq 0$ and $k \neq \infty$ ) can only exist at $\epsilon_{\infty}$ in the thermodynamic limit. Hence, in finite systems, regardless which value of $k$ we use, the series $\epsilon_{L, k}$ will always approach $\epsilon_{\infty}$ as $L$ increases. However, the rate of convergence probably will depend on $k$, and is fastest for some special value $k=k_{\text {opt }}$.

In order to measure the series $\epsilon_{L, k}$ a practical prerequisite is that the peaks in $P_{L, \epsilon}(E)$ are sufficiently well separated. The areas of the peaks are then calculated by

$$
W_{N}=\int_{-\infty}^{E_{\mathrm{cut}}} P_{L, \epsilon}(E) d E, \quad W_{I}=\int_{E_{\mathrm{cut}}}^{0} P_{L, \epsilon}(E) d E
$$

with the cutoff energy $E_{\text {cut }}$ defined as the energy between the two peaks where we split the peak areas apart. In fact $E_{\text {cut }}$ does not need to be particularly well-defined as the values of the states around $E_{\text {cut }}$ contribute very little to the total peak areas [106]. We have chosen $E_{\text {cut }}$ to be the average $E_{\text {cut }}=\int E P_{L, \epsilon}(E) d E$, with $P_{L, \epsilon}(E)$ obtained at the inverse temperature where the peak heights are equal, as in Fig. 4.2. The value of $E_{\text {cut }}$ is kept fixed while numerically solving Eq.(4.8).

The behavior of $\epsilon_{L, k}$ for 3D systems with $p=5,10,20$ is shown in Fig. 4.7, and for $2 \mathrm{D}$ systems with $p=20,35,50$ in Fig. 4.8, each time for three different values of $k$. In all cases the data show that $\epsilon_{L, k}$ approaches $\epsilon_{\infty}$ obtained via Eq. (4.2) regardless 
4 Finite-size scaling of the isotropic-nematic transition
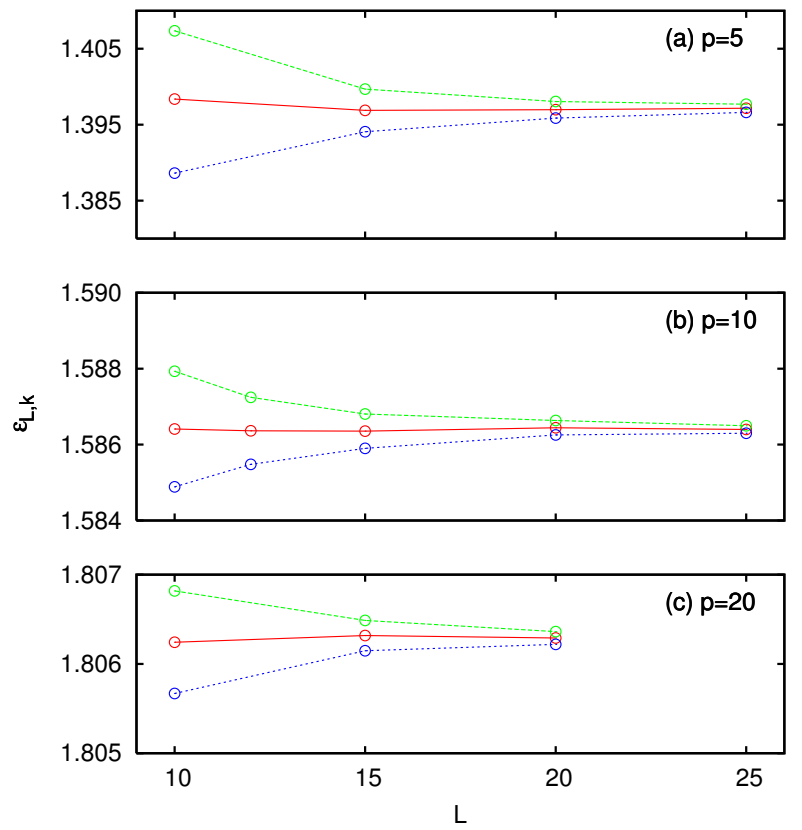

Figure 4.7: Variation of $\epsilon_{L, k}$ versus $L$ for 3D lattices using $p=5,10,20$. The lines are merely to guide the eye. The central data set in each subplot represent $\epsilon_{L, k_{\mathrm{opt}}}$ and show minimal finite-size effects. The upper and lower data in each subplot are calculated using $k_{\text {opt }} \pm 5 \Delta k$, with the uncertainty $\Delta k$ obtained following the method described in the text. 
4.4 Results and analysis

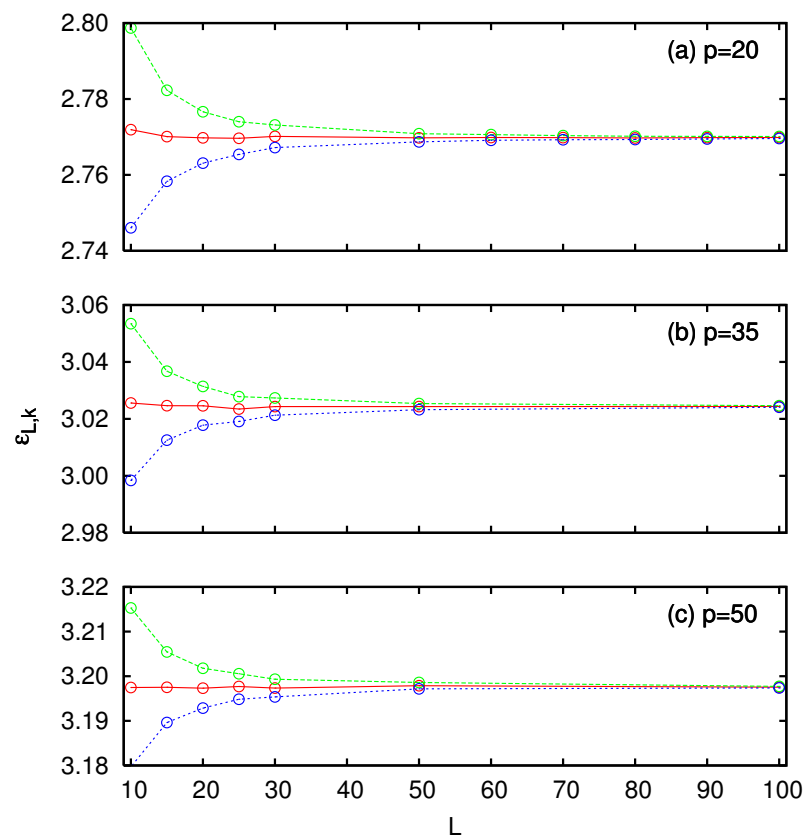

Figure 4.8: Variation of $\epsilon_{L, k}$ versus $L$, similar to Fig. 4.7, but for 2D lattices and using $p=20,35,50$. 


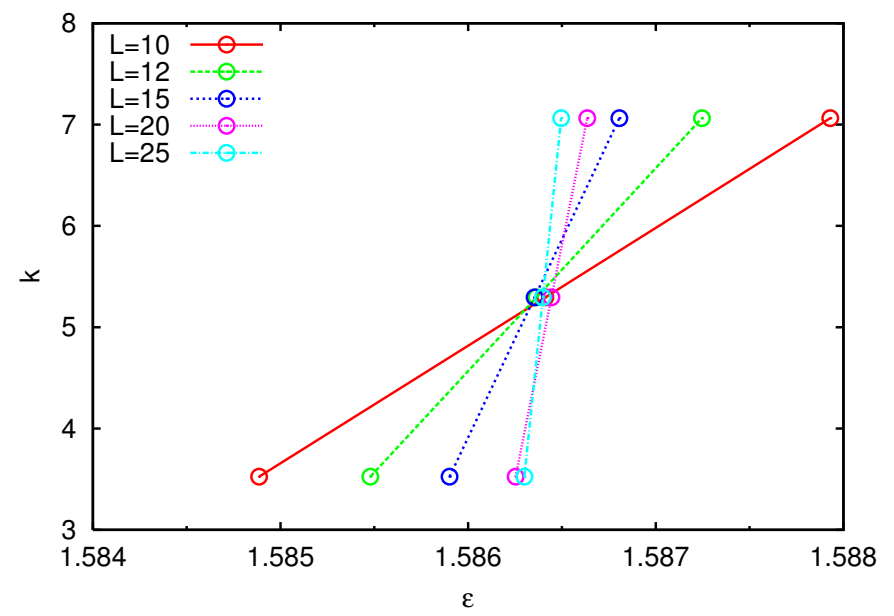

Figure 4.9: Calculation of $\epsilon_{\infty}$ using system sizes $L=10,12,15,20,25$ with $p=10$ on $3 \mathrm{D}$ lattices. The data points for each system size are at $k_{\mathrm{opt}}, k_{\mathrm{opt}}+5 \Delta k$ and $k_{\mathrm{opt}}-5 \Delta k$. The lines are to guide the eye. Despite $\Delta k$ being large in relation to $k$ the estimate of $\epsilon_{\infty}$ by this method is very accurate.

of the value of $k$, as predicted. For large $k$, where the ratio is dominated by the nematic peak area, $\epsilon_{\infty}$ is approached from high $\epsilon$. The converse is also true, with $\epsilon_{\infty}$ being approached for small $k$ from low $\epsilon$. We can thus indeed find a value $k=k_{\text {opt }}$, where finite-size effects are minimal.

To obtain an estimate of the uncertainties in $k_{\mathrm{opt}}$ and the transition inverse temperatures $\epsilon_{\infty}$, we compare two systems of different sizes $L_{i}$ and $L_{j}$. At some inverse temperature $\epsilon_{i j}$ both systems have the same ratio of peak areas $k_{i j}$. We take the average and the root mean fluctuations of $\epsilon_{i j}$ and $k_{i j}$ for all system size pairs and calculate $\epsilon_{\infty}$ and $k_{\text {opt }}$ along with their respective root-mean-square uncertainties from these values. As can be seen in Fig. 4.9, for $p=10$ on 3D lattices, the uncertainty in $k_{\text {opt }}$ is substantial but this does not prevent accurate estimates of $\epsilon_{\infty}$. The values of $\epsilon_{\infty, k}\left(\epsilon_{\infty}\right.$ for this method), $\ln k_{\text {opt }}$, and their respective uncertainties are given in Table 4.1 for 3D systems, and in Table 4.2 for 2D systems.

For non-optimal values of $k$ the shift in $\epsilon_{L, k}$ to $\epsilon_{\infty}$ is

$$
\epsilon_{\infty}-\epsilon_{L, k} \propto 1 / L^{d}
$$

i.e. it vanishes as a power-law in the inverse volume, as shown in Fig. 4.10, similar to Eq. (4.2). At $k=k_{\text {opt }}$ we find that the finite-size effects in $\epsilon_{L, k}$ are too small for a meaningful fit to be performed. This confirms that the method converges to $\epsilon_{\infty}$ quicker than $1 / L^{d}$. However, confirming that the convergence given by $k_{\mathrm{opt}}$ is exponential, as for the Potts model [95], still requires more accurate data. 


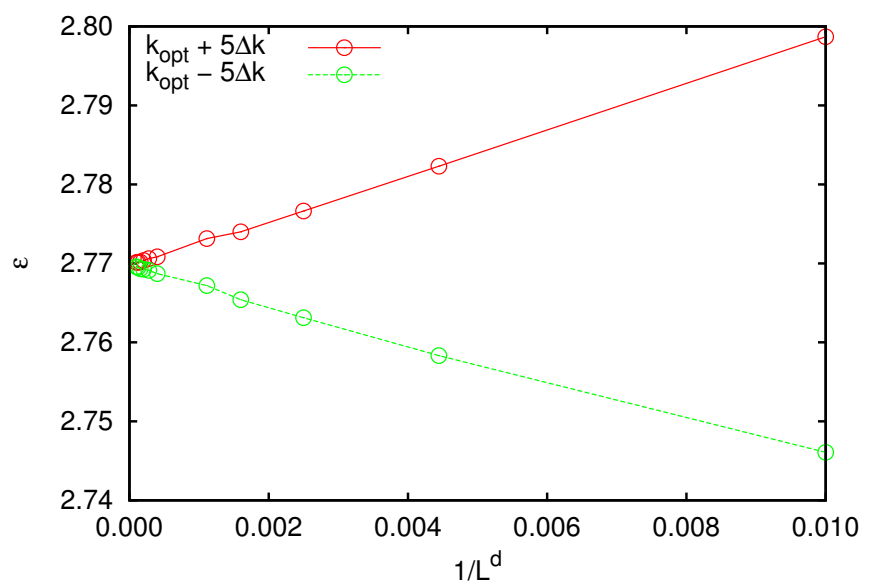

Figure 4.10: Scaling of non-optimal values of $k\left(k_{\mathrm{opt}}+5 \Delta k, k_{\mathrm{opt}}+5 \Delta k\right)$ with $1 / L^{d}$ using $p=20$ in $2 \mathrm{D}$. The scaling behavior is similar to Eq.4.2 but slower than for an optimally chosen value of $k$.

\section{A more practical implementation of the Borgs-Kotecký method}

An equivalent method to that described above is to plot the area ratio $k$ against $\epsilon$ for several system sizes [106, 107]. The phase transition should be exhibited by an intersection of these curves, taking place at $\epsilon=\epsilon_{\infty}$. Additionally, the value of $k$ at the intersection yields $k_{\text {opt }}$. This is shown for a $3 \mathrm{D}$ system with $p=10$ in Fig. 4.11. Obviously, the values of $\epsilon_{\infty}$ and $k_{\text {opt }}$ obtained from Fig. 4.11 coincide with the estimate listed in Table 4.1.

\subsubsection{Latent heat density}

We have seen that finite-size scaling relations that have originally been derived for first-order transition in the Potts model work well for the Lebwohl-Lasher model also. In agreement with previous simulations of the Lebwohl-Lasher model [99, 80], we have confirmed the validity of Eq.(4.2). We have also shown that the prediction of Refs. [100, 98, 101] holds, namely of finite-size effects vanishing faster than $1 / L^{d}$ by choosing an appropriate ratio of peak areas $k=k_{\text {opt }}$. In the Potts model, it has additionally been shown that the number of Potts states $q=k_{\text {opt }}$, i.e. finite-size effects are minimized when the ratio of the areas in the order parameter distribution is set equal to $q$.

We now verify that an analogous prediction holds for the Lebwohl-Lasher model, but with $q$ replaced by $k_{\text {opt }}$. Even though the Lebwohl-Lasher model is a continuous spin model, Eq. 4.2 allows us to obtain an effective number of spin states by fitting 
4 Finite-size scaling of the isotropic-nematic transition

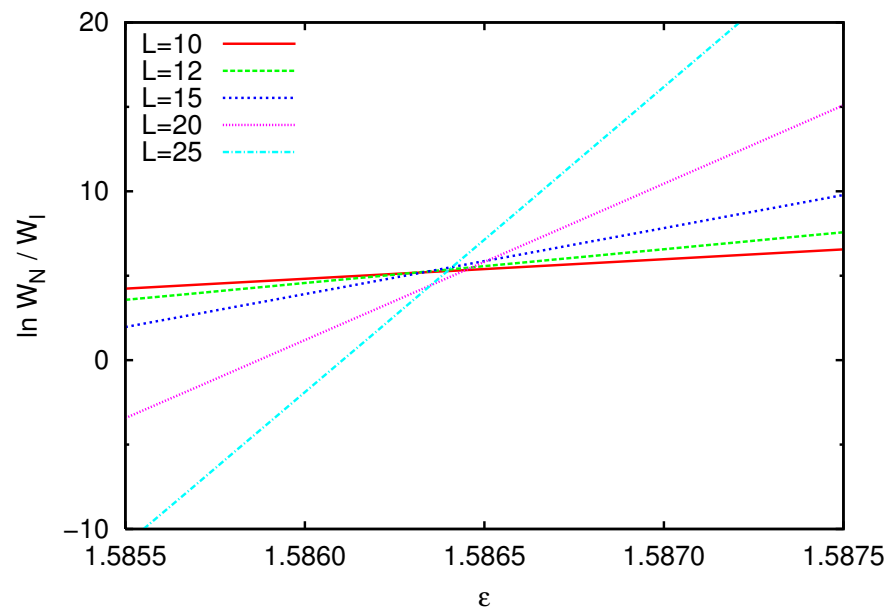

Figure 4.11: $\ln W_{N} / W_{I}$ versus $\epsilon$, with $p=10$ for $L=10-25$ on $3 \mathrm{D}$ lattices. The intersection gives $\ln k_{\mathrm{opt}} \approx 5.3$ at $\epsilon_{\infty} \approx 1.5864$.

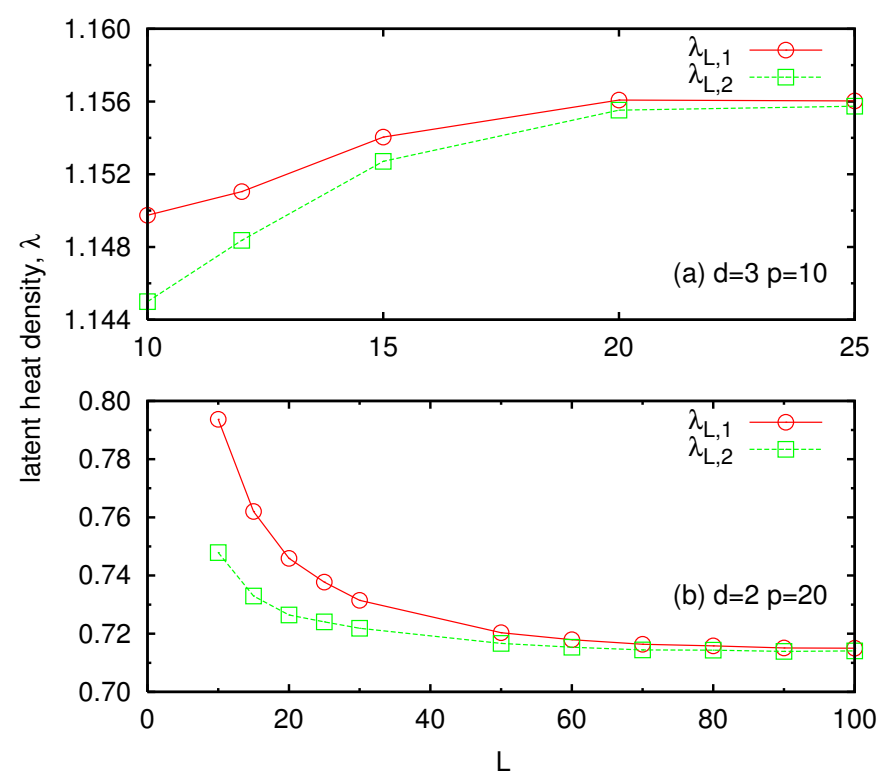

Figure 4.12: Variation in the latent heat density estimators $\lambda_{L, i}$ with system size. (a) The latent heat estimators for $p=10$ on 3D lattices converging to $\lambda_{\infty}$ from below. (b) The estimators for $p=20$ on 2D lattices converge to $\lambda_{\infty}$ from above. 
to finite-size simulation data and using

$$
A_{\text {Potts }}=\ln q / \lambda_{\infty}
$$

where $\lambda_{\infty}$ is the latent heat density. To check whether Eq.44.11) holds for the Lebwohl-Lasher model we obtain $\lambda_{\infty}$ independently by two methods. Firstly, the latent heat is related to the specific heat maximum 98

$$
\lambda_{L, 1}=\sqrt{\frac{4 c_{L, \max }}{L^{d}}}
$$

which should approach $\lambda_{\infty}$ as $L \rightarrow \infty$. Secondly, the latent heat can be read directly from the order parameter distribution as the peak-to-peak distance, marked $\Delta \rho$ in Fig. 4.2. This can also be written numerically as

$$
\lambda_{L, 2}=\frac{2\langle|E-\langle E\rangle|\rangle}{L^{d}} .
$$

To obtain $\lambda_{\infty}$, we measure $\lambda_{L, i}(i=1,2)$ for several system sizes $L$ and extrapolate in $L$ using $\lambda_{\infty}-\lambda_{L, i} \propto 1 / L^{d}$. Some typical results are shown in Fig. 4.12 ; both estimators tend towards a common value for $\lambda_{\infty}$ with minimal difference between the two estimators for large systems. A particular feature observed is that $\lambda_{\infty}$ is approached from below in $3 \mathrm{D}$, but from above in $2 \mathrm{D}$. If enough $2 \mathrm{D}$ systems were stacked atop one-another, we would expect a crossover from the latter to the former behavior.

We now use the estimates of $\lambda_{\infty}$ and $k_{\text {opt }}$ to calculate the ratio $\ln k_{\text {opt }} / \lambda_{\infty}$; results are listed in Table 4.1 for $3 \mathrm{D}$ systems and in Table 4.2 for $2 \mathrm{D}$ systems. The relation $\ln k_{\text {opt }} / \lambda_{\infty} \sim A_{\text {Potts }}$ is found to hold in all cases, albeit with fairly large numerical uncertainty. Hence, even though the Lebwohl-Lasher model is a continuous model, its finite-size scaling behavior is well described by the Potts model, with an effective number of Potts states $q_{\mathrm{eff}}=k_{\mathrm{opt}}$.

\subsection{Summary}

In agreement with other works [99, 80], we have shown that extrapolation of the finite-size inverse temperature of the specific heat maximum $\epsilon_{L, c}$ converges to $\epsilon_{\infty}$ with a shift vanishing as $A_{\text {Potts }} / L^{d}$, similar to the first-order phase transitions of the Potts model. Because of this similarity we analysed another method used to locate the transition inverse temperature $\epsilon_{\infty}$ for the Potts model, where finite-size effects vanish exponentially. To this end, we defined the estimators $\epsilon_{L, k}$, which are the finite-size inverse temperatures where the ratio of nematic to isotropic peak areas in the order parameter distribution is equal to $k$. We indeed found that the series $\epsilon_{L, k}$ converges faster with $L$ to $\epsilon_{\infty}$ than $1 / L^{d}$, provided an optimal value $k=k_{\text {opt }}$ is used. An advantage of this method is that only moderate system sizes 
are required, because the system size dependence of $\epsilon_{L, k}$ is very weak for $k=k_{\text {opt }}$. Furthermore we noticed that the ratio $k_{\text {opt }} / \lambda_{\infty}$, where $\lambda_{\infty}$ is the latent heat density in the thermodynamic limit, is remarkably similar to the proportionality constant $A_{\text {Potts }}$ of the scaling of $\epsilon_{L, c}$ (Eq. (4.2)). We therefore conclude that finite-size scaling predictions for first-order transitions in the Potts model can also be used for the first-order isotropic-nematic transition of the Lebwohl-Lasher model, provided that $k_{\text {opt }}$ is replaced by $q$.

Despite it being stated that it would be difficult to extend the derivation of the finite-size scaling relations from the Potts model to continuous spin models [101, it is perhaps not so surprising that this $q \rightarrow k_{\text {opt }}$ swap works. If the exponent $p$ in the Hamiltonian of the Lebwohl-Lasher model (Eq.4.4) is made very large, the interaction becomes increasingly like that of a Potts model, as $\lim _{p \rightarrow \infty}\left|\vec{d}_{i} \cdot \vec{d}_{j}\right|^{p}=$ $\delta\left(\vec{d}_{i}, \vec{d}_{j}\right)$. In this case only very well-aligned neighboring spins lower the energy appreciably, nearing the pair interaction of the Potts model. 


\section{Confinement of nematic liquid crystals}

\section{Contents}

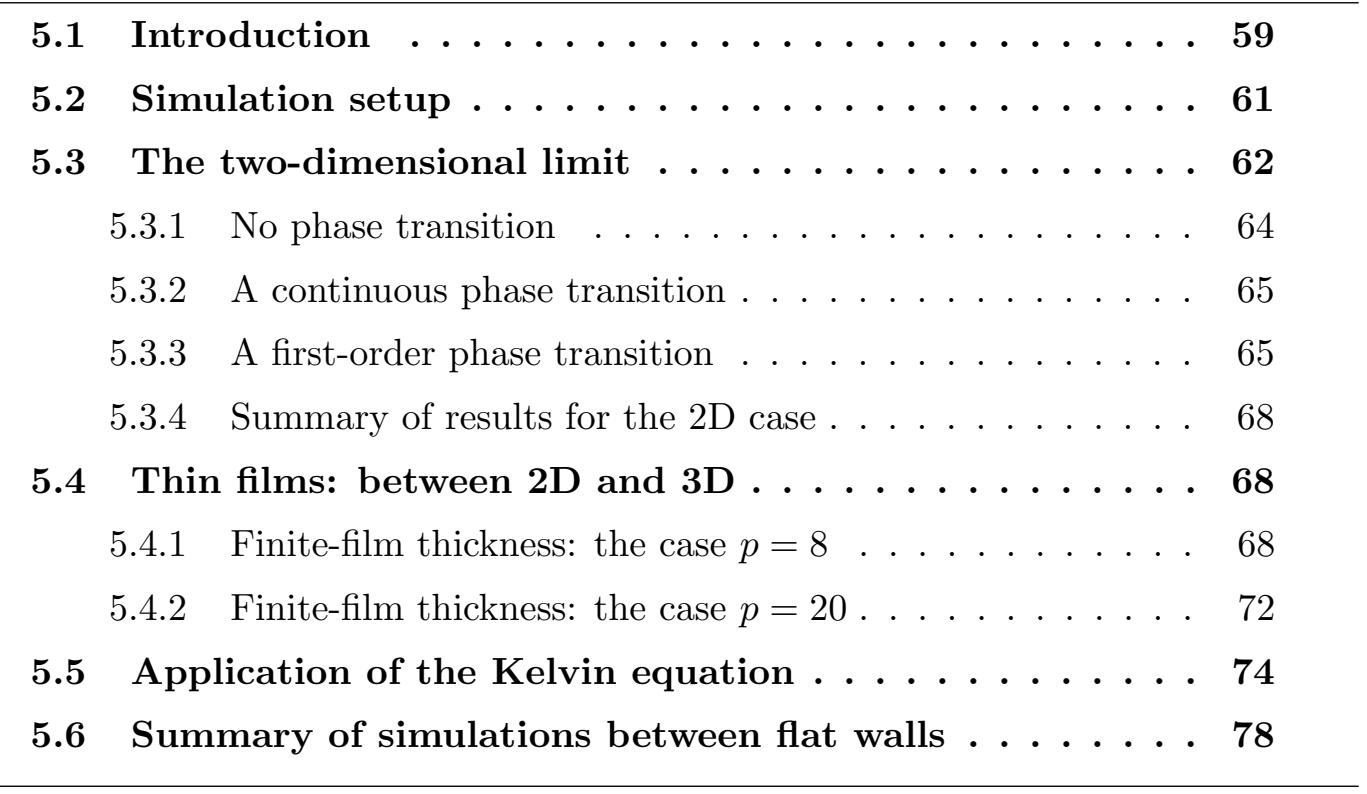

\subsection{Introduction}

It is standard for the isotropic-nematic phase transition of liquid crystals in bulk (i.e. in $d=3$ dimensions in the absence of any disorder or confining surfaces) to be first-order and for there to be long-range order in the nematic phase. In the isotropic phase the system is rotationally invariant; this invariance is broken in the nematic phase, hence the first-order phase transition [27], see also chapter 1 .

When a liquid crystal is confined between two parallel plates the first-order phase transition need not occur. As the inter-plate distance $H$ becomes small the isotropicnematic phase transition is thought to become continuous [108, 109, 110, 111, 112, 113. Simulations show that the first-order phase transition is expected to become continuous below some thickness $H_{x}$ [114, 115, 116, 117, 118.

As $H \rightarrow 1$ the system becomes two-dimensional (2D). There appears to be not only many possibilities but also confusion regarding phase transitions in this limit. 
5 Confinement of nematic liquid crystals

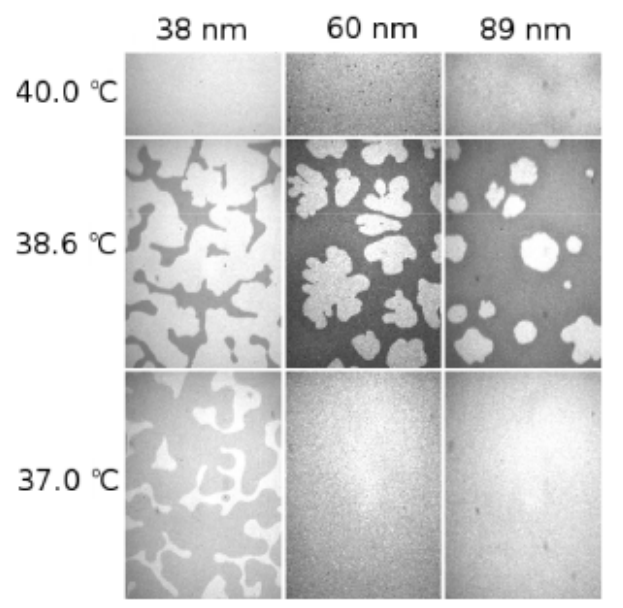

Figure 5.1: Experimental evidence of liquid crystal thin-films exhibiting phase coexistence at the isotropic-nematic phase transition. Shown are micrographs of 38,60 and 89 nm-thick films of 4-n-octyl-4'-cyanophenyl (8CB) at temperatures $37.0,38.6$ and $40.0{ }^{\circ} \mathrm{C}$. The micrograph of the $89 \mathrm{~nm}$ film in the row labeled $38.6{ }^{\circ} \mathrm{C}$ is actually taken at $39.5{ }^{\circ} \mathrm{C}$. Reprinted figure with permission from Ref. [31].

Recent simulations of the Lebwohl-Lasher model 64] have come to the conclusion that either no phase transition exists [119, 120], or a phase transition with KosterlitzThouless [121] characteristics occurs [122]. In contrast, continuous phase transitions have been very difficult to realize experimentally [109, 29]. Instead, experiments in 2D have shown coexistence of large isotropic and nematic domains [29, 30, 31], indicating a first-order phase transition, see Fig. 5.1. This seems somewhat strange as the Mermin-Wagner theorem states that confinement to $d \leq 2$ dimensions destroys long-range order in the nematic phase [62]. However, quasi-long-range order is permissible and correlations will then observe power-law decay, but the decay may be very slow [48]. Additionally, there exists a mathematically rigorous proof showing that first-order isotropic-nematic phase transitions are in fact possible in 2D [123, 87], and this has been confirmed by simulations [124, 125].

In short, from this jumble of scenarios we can confidently say that the isotropicnematic phase transition in confinement cannot belong to a single universality class but instead must be dependent on microscopic details.

This confusion is the motivation of this chapter to find one possible microscopic detail responsible for determining which thin-film scenario occurs. In this chapter it is shown that a generalized Lebwohl-Lasher model can reproduce all the thin-film scenarios described above, with the change of just one parameter in the Hamiltonian. By making the pair-interaction sharper and more narrow (i.e. a stronger interaction between aligned particles, but an indifferent one for unaligned particles, see section 


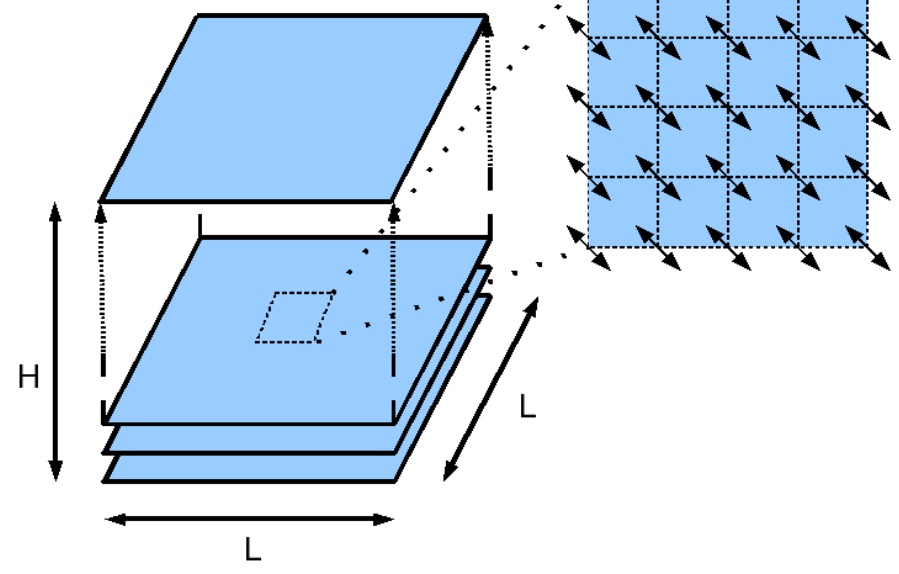

Figure 5.2: Image of the simulation setup. Spins are attached at regular lattice points on each layer. We use periodic boundary conditions in the $L$ direction, but not in the $H$-direction to describe the planar confinement. For $H=1$, the lattice is purely two-dimensional, corresponding to a single layer of spins; increasing $H \rightarrow \infty$ creates a three-dimensional system.

3.4), we can move from no phase transition in the $2 \mathrm{D}$ limit, to a continuous phase transition, to a first-order isotropic-nematic phase transition. This last case is particularly interesting as it matches experiments (first-order phase transitions in 2D with phase coexistence, see Fig. 5.1) and has not previously been realized in the 2D limit by simulations.

The outline of this chapter is as follows. In section 5.2, we describe the simulation model. Next, in section 5.3, we present results for purely 2D liquid crystals. In section 5.4 we then enlarge the simulation box into the third dimension to create "thin films", and analyse the differences with the purely 2D limit. In section 5.5 we analyse the first-order phase transitions observed and perform a test of the Kelvin equation, i.e. the shift of the transition temperature as a function of film thickness. We believe this is important as it verifies that the transitions we observe are genuinely first-order, and not just finite-size artifacts. Finally, in section 5.6, we summarize our findings.

\subsection{Simulation setup}

The original Lebwohl-Lasher model [64] (described in detail in chapter 3) is defined on a three-dimensional (3D) periodic lattice of form $V=L \times L \times L$, with volume $V$ and system side length $L$. A $3 \mathrm{D}$ unit vector $\vec{d}_{i}$ is placed on each lattice site $i$ and 
these interact with their nearest neighbors at sites $j$ via

$$
\mathcal{H}=-\epsilon \sum_{\langle i j\rangle}\left|\vec{d}_{i} \cdot \vec{d}_{j}\right|^{p}
$$

with exponent $p=2$ and coupling constant $\epsilon$. The $\langle i j\rangle$ denotes the sum over nearest neighbors. The factor $1 / k_{B} T$ is incorporated into the coupling constant, with $k_{B}$ the Boltzmann constant and $T$ the temperature, so that $\epsilon>0$ is playing the role of the inverse temperature. In this form the model undergoes a first-order phase transition, as was already discussed previously [77, 102, 80, 65, 126] at $\epsilon \approx 1.34$ [103.

In chapter 4 we made a generalization to Eq.(5.1) by altering the value of $p$. In order to study planar confinement, we remove the periodic boundary condition in one direction, forming a "sandwich" geometry, representative of a thin-film. The simulation box can now be described as being $V=L \times L \times H$, with periodic boundary conditions in the $L$ directions but not in the $H$ direction. This setup is shown in Fig. 5.2. In this sandwich geometry, the thermodynamic limit is obtained by letting $L \rightarrow \infty$ at fixed $H$. This confined Lebwohl-Lasher model setup is identical to that of a previous study 114. By setting $H=1$, we form a purely $2 \mathrm{D}$ system (although the vectors $\vec{d}_{i}$ are still chosen to be $3 \mathrm{D}$ ).

\subsection{The two-dimensional limit}

It is now our aim to show that the three scenarios for the isotropic-nematic transition in confinement described in the introduction can all occur in this $2 \mathrm{D}$ limit, namely:

1. no transition occurs;

2. a continuous transition occurs;

3. a first-order transition occurs.

We do this by changing only the exponent $p$ in Eq. 5.1). Again, in the 2D limit, the film thickness $H=1$, while the lateral extensions of the box $L \rightarrow \infty$, see also Fig. 5.2 .

The simulation method used throughout this section is the same as in chapter 4. That is, we use transmission matrix Wang-Landau sampling see 2.4.2 to obtain the distribution $P(\rho, S)$, defined as the probability to observe the system in a state with energy density $\rho$ and nematic order parameter $S$. Recall that the latter is defined as the maximum eigenvalue of the orientational tensor, see section 1.4.1, and that it is intensive: $0 \leq S \leq 1$. 

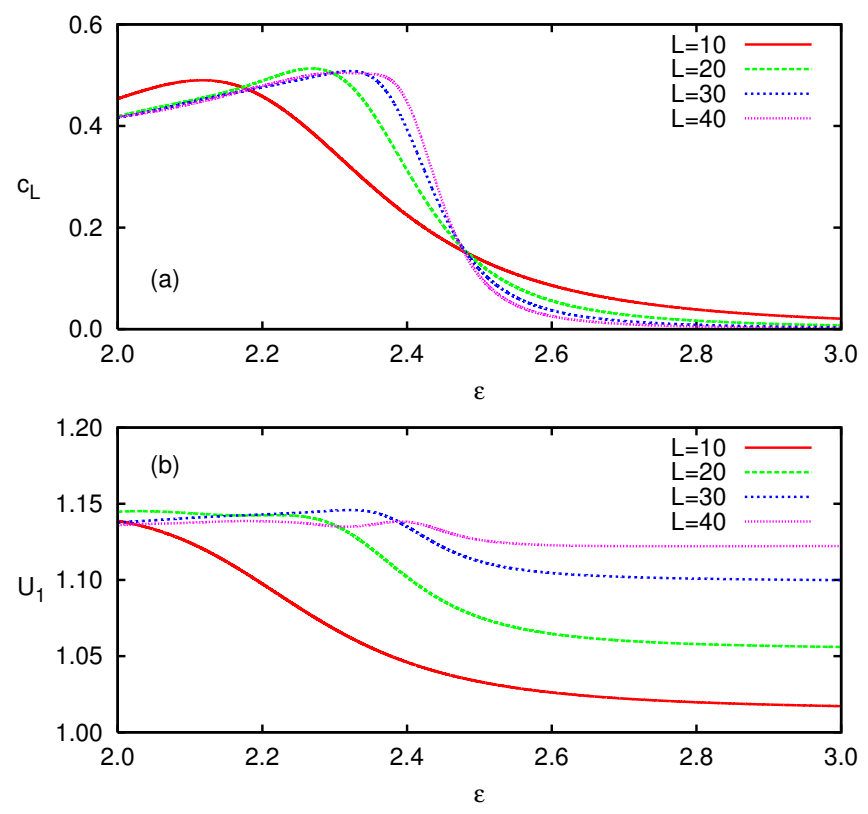

Figure 5.3: Finite-size scaling analysis of the model of Eq.5.1 for $p=2$ in the 2D limit, i.e. the film thickness $H=1$, while the lateral extensions $L$ are varied. Shown in (a) is the specific heat $c_{L}$ versus inverse temperature $\epsilon$ for various system sizes $L$. The peaks shift with $L$ to larger values of $\epsilon$. Hence, if a transition takes place, it must occur at some value $\epsilon>2.35$, as this is where we observe the specific heat maximum of the $L=40$ system. Shown in (b) is the cumulant $U_{1}$ versus $\epsilon$ for various $L$. The key message is that the cumulant does not progress with system size towards an ordered phase at high- $\epsilon$. If the phase transition were to occur the $U_{1} \rightarrow 1$ for the larger systems at high- $\epsilon$ (compare, for instance, to Fig. 5.4(a) where a cumulant intersection does occur). 


\subsubsection{No phase transition}

Let us start with the exponent in Eq.(5.1) set to $p=2$. Although this has been extensively studied, even recent papers have disagreed with each other, predicting either a phase transition possibly of the Kosterlitz-Thouless type [122] or no phase transition at all [119, 120]. Here, we briefly revisit the problem using our own data.

To this end, we consider the cumulant $U_{1}=\left\langle S^{2}\right\rangle /\langle S\rangle^{2}$. As is well known, $U_{1}$ becomes independent of system size at a continuous phase transition [92, 127]. This property is intrinsically related to hyperscaling. To show the $L$-independence of the cumulant, one expresses the order parameter $\langle S\rangle$ and susceptibility $\chi=$ $V\left(\left\langle S^{2}\right\rangle-\langle S\rangle^{2}\right)$ in terms of the correlation length $\xi$ :

$$
\langle S\rangle \propto \xi^{-\bar{\beta}}, \quad \chi \propto \xi^{\bar{\gamma}} .
$$

These expressions hold at both a second order phase transition, as well as at a Kosterlitz-Thouless transition [128]. Note that, at a second order transition, the exponent ratios can furthermore be expressed as $\bar{\beta}=\beta / \nu$ and $\bar{\gamma}=\gamma / \nu$, with $\nu$ the correlation length critical exponent. At a Kosterlitz-Thouless transition, this identification cannot be made, since then the correlation length diverges faster than any power law in temperature; the exponent $\nu$ itself is then not defined, but exponent ratios still are.

In terms of the order parameter and susceptibility, the cumulant becomes

$$
U_{1}-1=\frac{\chi}{V\langle S\rangle^{2}} \propto \xi^{\bar{\gamma}+2 \bar{\beta}-d}
$$

where in the last step Eq. (5.2) was used. If we now use the finite size scaling Ansatz $\xi \propto L$, as well as the hyperscaling relation

$$
\bar{\gamma}+2 \bar{\beta}-d=0
$$

it follows that the $L$-dependence in Eq. (5.3) vanishes, showing that the cumulant is system size independent at a continuous transition. A continuous transition may thus be located by plotting $U_{1}$ versus the inverse temperature $\epsilon$ for a number of system sizes $L$ : at the transition temperature, curves for different $L$ intersect.

To test this idea, we first plot in Fig. 5.3(a) the specific heat $c_{L}=\left(\left\langle E^{2}\right\rangle-\langle E\rangle^{2}\right) / V$ versus inverse temperature $\epsilon$, for a number of system sizes, where $E$ denotes the energy given by Eq.(5.1). The salient feature is a maximum, which shifts to larger $\epsilon$ as $L$ increases. We conclude that, if a transition occurs, the transition inverse temperature of the thermodynamic limit must occur at some value $\epsilon>2.35$, since this is where the specific heat maximum of the $L=40$ system occurs. However, in that regime, there is no sign of an intersection in the cumulant, see Fig. 5.3(b). Rather, as $L$ increases, the cumulant approaches the same value as in the high-temperature region, from which we conclude that the presence of a transition is unlikely. Hence, our analysis agrees best with the recent findings of Paredes [119, 120]. 


\subsubsection{A continuous phase transition}

We have seen in chapter 4 that increasing the value of $p$ in Eq. (5.1) makes the pairinteraction sharper; in three spatial dimensions this made the isotropic-nematic transition more strongly first-order. By repeating this idea, we show that it is possible to induce a phase transition in two dimensions also, simply by increasing the exponent $p$. To this end, we now choose $p=8$ (recall from the previous section that for $p=2$ no transition was observed).

Evidence for a continuous phase transition is shown in Fig. 5.4 (a), as the $U_{1}$ curves for various $L$ now intersect, this being at $\epsilon(H=1) \approx 2.452$. Further evidence that the transition is continuous, as opposed to first-order, follows from the scaling of the specific heat $c_{L}=\left(\left\langle E^{2}\right\rangle-\langle E\rangle^{2}\right) / V$, where $V=H L^{2}$ is the number of lattice sites. In Fig. 5.4 (b), we plot $c_{L}$ versus the inverse temperature $\epsilon$, and we observe a maximum that increases with $L$. At a first-order phase transition, the specific heat maximum scales with the volume of the system, i.e. $c_{L, \max } \propto V$. For a 2D system this implies

$$
c_{L, \max } \propto L^{\bar{\alpha}}
$$

with $\bar{\alpha}_{1 s t}=2$. If we apply this scaling relation to the maxima in Fig. 5.4 (b) we obtain $\bar{\alpha}=0.379$, implying that we are observing a continuous and not a first-order phase transition.

In Fig. 5.4 (c), we plot the susceptibility versus $\epsilon$. As expected at a continuous transition, the susceptibility for the finite system reveals a maximum, which grows rapidly with $L$. As a precaution, we check that the hyperscaling relation Eq. (5.4) has been fulfilled at $\epsilon=2.452$. By using Eq. (5.2) together with the scaling Ansatz $\xi \propto L$, we obtain $\bar{\beta} \approx 0.19$ and $\bar{\gamma} \approx 1.63$ at this temperature, which agrees rather well with the hyperscaling equation. The values of the critical exponents are quite different to the values of the $X Y$ universality class, $\bar{\beta}_{X Y}=0.125$ and $\bar{\gamma}_{X Y}=1.75$ [128]. This indicates that the phase transition observed belongs to a different universality class.

\subsubsection{A first-order phase transition}

As the $p=8$ system undergoes a phase transition, it would be natural to assume that a $p=20$ system does likewise. Indeed, in Fig. 5.5(a) we observe an intersection of $U_{1}$ curves at $\epsilon=2.770$. However, this time the phase transition is first-order, and not continuous.

As explained in section 4.4.1, at a first-order phase transition, a free energy barrier $\Delta F$ arises, which reflects the cost of the interface between ordered (quasi-nematic) and disordered (isotropic) domains. This barrier can be "read-off" as the height of the peaks in the energy density probability distribution $\ln P(\rho)$, and it scales with the size of the system as $\Delta F \propto L^{d-1}$, where $d$ is the spatial dimension (see also Fig. 5.10). In the present case $d=2$, and from Fig. 5.5(b) we indeed see that $\Delta F$ increases linearly with the system size. This confirms that, using $p=20$, the system indeed undergoes a first-order phase transition. 

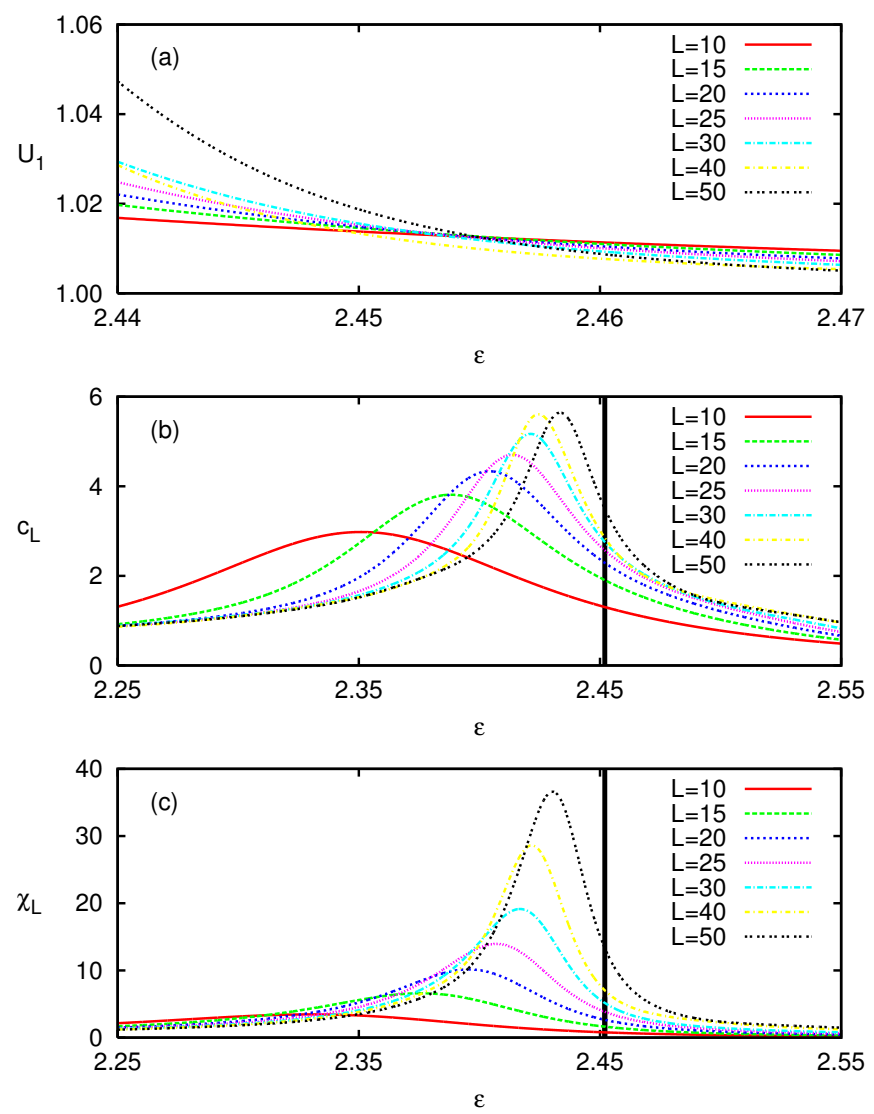

Figure 5.4: Data for Eq. 5.1) with $p=8$ in 2D, i.e. $H=1$. In (a) we plot $U_{1}$ against inverse temperature $\epsilon$ for many system sizes $L$. We observe an intersection point of these curves, indicating a phase transition. The specific heat $c_{L}$ and susceptibility $\chi_{L}$ against inverse temperature are shown for many $L$ in (b) and (c) respectively. The thick vertical lines at $\epsilon=2.452$ in (b) and (c) indicate where the inverse temperature of the cumulant intersection occurs. The critical exponent ratios are calculated using Eq. (5.2) at this inverse temperature. 
5.3 The two-dimensional limit
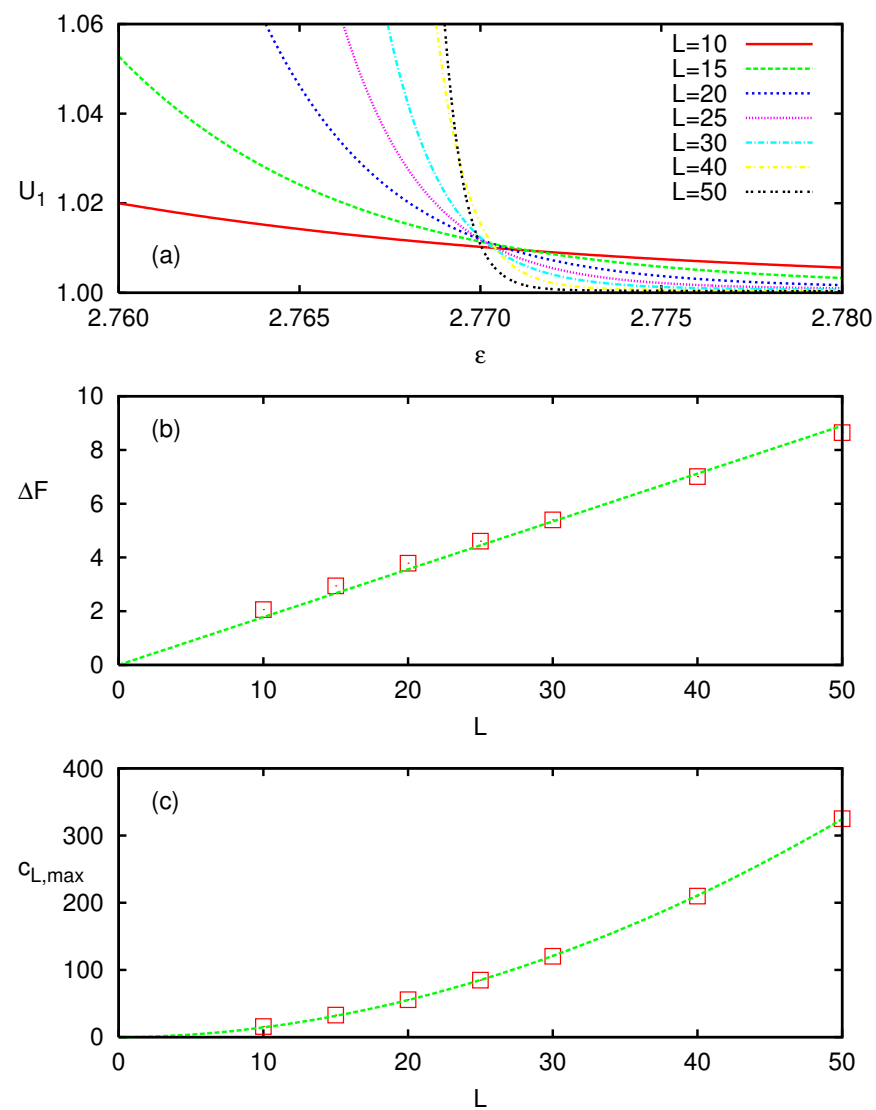

Figure 5.5: Data for $p=20$ in 2D, i.e. $H=1$. We observe an approximate intersection of the curves for various $L$ in the $U_{1}$ versus $\epsilon$ plot (a). The linear increase of $\Delta F$ with $L$ in (b) and the quadratic increase of $c_{L, \text { max }}$ with $L$ in (c) indicate that the phase transition is first-order. 
5 Confinement of nematic liquid crystals

Additionally, in Fig. 5.5(c) we see that the specific heat maxima increase $c_{L, \max } \propto$ $L^{\bar{\alpha}}$, with $\bar{\alpha} \approx 1.98$ obtained by fitting. This is very close to $\bar{\alpha}_{1 s t}=2$ of a first-order phase transition in 2D and provides further confirmation that the transition is now first-order.

\subsubsection{Summary of results for the $2 \mathrm{D}$ case}

By analyzing the generalized Lebwohl-Lasher model using three different exponents $p$ in Eq.(5.1), we have demonstrated that a single scenario describing the isotropicnematic transition in 2D does not exist. A simple change in microscopic detail, namely the value of $p$, determines what type of phase transition takes place, or even if a phase transition takes place at all.

In 3D the isotropic-nematic transition in Eq. 5.1) is first-order for $p \geq 2$. In 2D a first-order transition requires a higher value of $p$. Since the system with $p=20$ undergoes a first-order phase transition in $2 \mathrm{D}$ we expect the phase transition to be first-order irrespective of the film thickness $H$. In contrast, for $p=8$ the transition is continuous in $2 \mathrm{D}$, which means that a cross-over to first-order behavior must occur as $H$ increases. Similarly, for $p=2$ we observe no transition in 2D, which means that a cross-over to first-order behavior also takes place upon increasing $H$. In the following section we look at the change in behavior of these models as the systems become thicker and the bulk limit is approached, i.e. as we let $H \rightarrow \infty$.

\subsection{Thin films: between 2D and 3D}

We now analyse different scenarios of the isotropic-nematic phase transition in thinfilm geometry by extending the thickness in the non-periodic dimension, i.e. we now consider a $L \times L \times H$ simulation box, with periodic boundary conditions in the $L$ directions, but not in the $H$ direction, and with $H>1$. The Lebwohl-Lasher model with $p=2$ has previously been studied with this setup and it was found that a first-order phase transition exists provided the film thickness exceeds a certain crossover value somewhere between $H_{x}=8-16$ lattice layers [114]. We here revisit the problem but using a larger exponent $p=8$ in Eq. (5.1).

\subsubsection{Finite-film thickness: the case $p=8$}

Based on our previous finding that the transition for $p=8$ is continuous in the limit $H=1$, we postulate there must be two identifiable regions in this case:

1. $H<H_{x}$ where the phase transition is continuous; and

2. $H>H_{x}$ where the phase transition is first-order. 

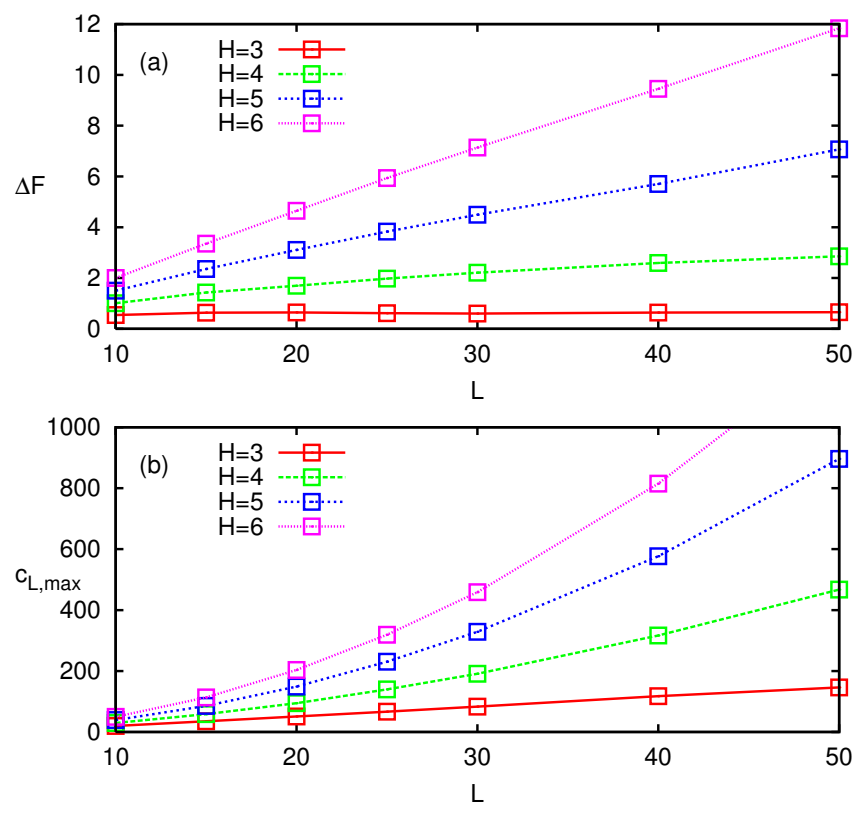

Figure 5.6: Evidence of the phase transition of the $p=8$ model becoming firstorder as the system becomes thicker. In (a) we see that the $\Delta F$ barrier has become proportional to $L$ by $H=6$. For $H=6$ the specific heat maximum also increases proportional to $L^{2}$, as seen in (b). In both plots the lines are merely to guide the eye. 
Table 5.1: Properties of the continuous isotropic-nematic phase transitions observed with $p=8$, i.e. for values of the film thickness below $H_{x}$. Listed are the transition inverse temperature $\epsilon_{\infty}(H)$, and the critical exponents of the nematic order parameter $\bar{\beta}$ and susceptibility $\bar{\gamma}$, for film thicknesses $1 \leq H \leq 4$.

\begin{tabular}{c|ccc}
$H$ & $\epsilon_{\infty}(H)$ & $\bar{\beta}$ & $\bar{\gamma}$ \\
\hline 1 & 2.452 & 0.19 & 1.63 \\
2 & 1.864 & 0.17 & 1.67 \\
3 & 1.716 & 0.15 & 1.71 \\
4 & 1.650 & 0.10 & 1.81 \\
\hline
\end{tabular}

To obtain the crossover thickness whereupon the phase transition becomes firstorder we consider the free energy barrier $\Delta F$ of interface formation, as well as the specific heat. As can be seen in Fig. 5.6(a), the barrier $\Delta F$ grows $\propto L$ at $H=5-6$. Additionally, in Fig. 5.6(b), at $H=6$, we obtain a value of $\bar{\alpha}=2.00$ for the fit of the specific heat maxima with system size $L$, see Eq.(5.5), confirming that the phase transition is first-order in these cases. For $H=5$ we obtain $\bar{\alpha}=1.94$ and for $H=4$ we obtain $\bar{\alpha}=1.74$. We therefore conclude that the phase transition is continuous for $H=4$ and that the crossover thickness is at $H_{x}=5$.

Using the cumulant intersection method, we can determine at what temperatures the continuous phase transitions take place for values of the film thickness $1 \leq H \leq$ 4. Once the critical temperatures have been located, we can use Eq. (5.2) to obtain the critical exponent ratios. These critical exponents are given in Table 5.1, along with the transition temperatures. For $H>1$ the general trend is for $\bar{\beta} \rightarrow 0$ and $\bar{\gamma} \rightarrow 2$, i.e. the ratios approach their first-order values.

Although we have discussed the scaling of the nematic order parameter $S$ at the transition point, Eq. (5.2), we have not yet looked at how $S$ scales at other temperatures. Shown in Fig. 5.7 are typical behaviors for (a) the bulk case, for (b) a first-order phase transition in a film of thickness $H=10$, and for (c) a continuous phase transition with $H=2$. In all cases $S$ increases with $\epsilon$ and the slope $d S / d \epsilon$ has a maximum at approximately $\epsilon_{\infty}(H)$. For the first-order phase transitions shown in Fig. 5.7, both in bulk (a) and also for the $H=10$ film (b), $S$ becomes independent of system size at high $\epsilon$. This suggests long-range nematic order, as otherwise $S \rightarrow 0$ in the thermodynamic limit. For the bulk case, the presence of long-range order is undebated. For the confined case $H=10$, the situation is less clear. In this case the Mermin-Wagner theorem [62] may still apply, but it could be that the decay of nematic order with system size $L$ requires system sizes beyond the reach of our simulations to be seen. 

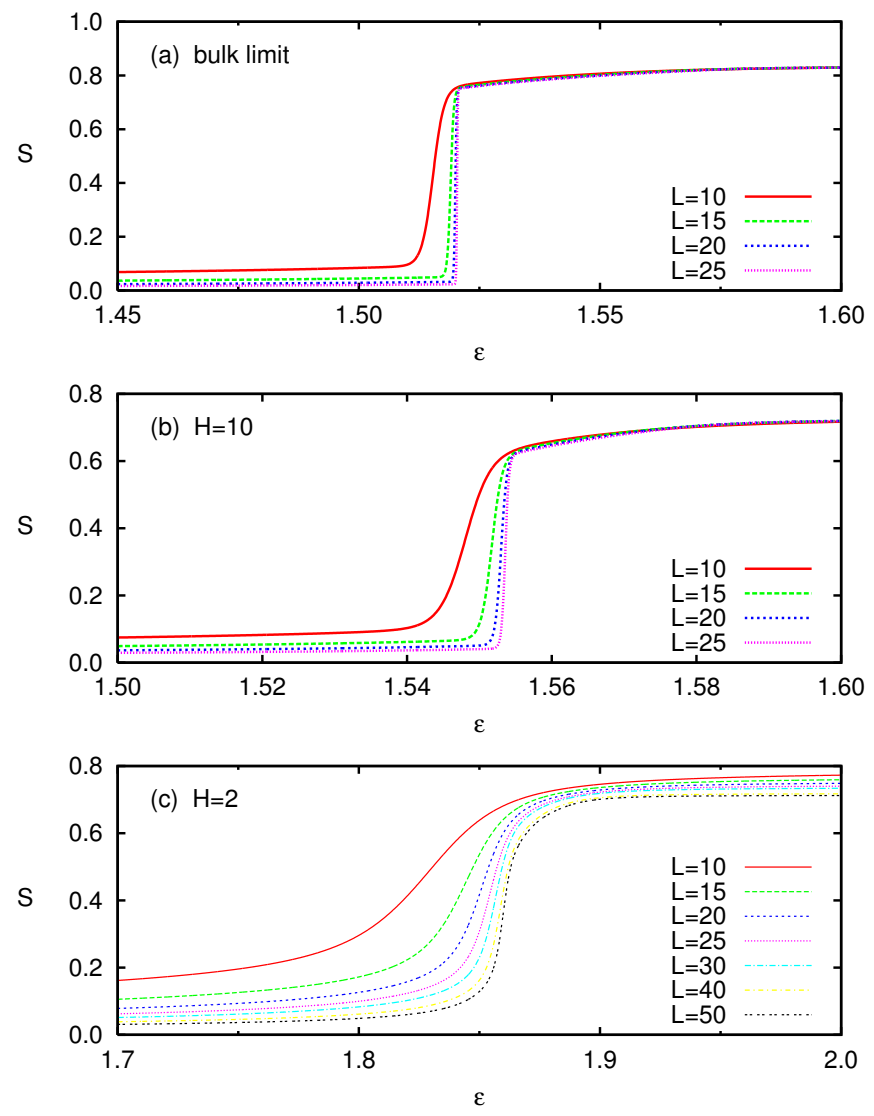

Figure 5.7: Nematic order parameter $S$ against inverse temperature $\epsilon$ for (a) bulk three-dimensions, (b) inside a film with thickness $H=10$ (still above $H_{x}$ ), and (c) a film with $H=2$, all using $p=8$. In contrast with the first-order phase transitions observed for (a) and (b), distinctly different behavior is observed at high $\epsilon$ for $H=2$ where the transition is continuous: $S$ decays with increasing $L$ in that case. 


\section{Confinement of nematic liquid crystals}

In contrast to the first-order transitions, the curves for the continuous phase transition in Fig. 5.7(c) do not saturate at high $\epsilon$ but instead decrease with $L$. This indicates the absence of long-range nematic order in the thermodynamic limit. Hence, the most likely explanation is that we see a transition with KosterlitzThouless characteristics in this case. One additional piece of evidence pointing to a Kosterlitz-Thouless type phase transition for $H<H_{x}$ is the behavior of the specific heat maximum. As seen in Fig. 5.4 (c), $c_{L, \max }$ barely grows with system size for $H=1$, consistent with a negative specific heat exponent, in turn consistent with a Kosterlitz-Thouless type phase transition [121]. For larger values of the film thickness $H$, we observe much faster growth of $c_{L, \max }$ with $L$, as can be seen in Fig. 5.6(b), ultimately scaling as $c_{L, \max } \propto L^{2}$ for $H=6$, where the transition is first-order.

In principle, for a Kosterlitz-Thouless transition, we could also obtain $\epsilon_{\infty}(H)$ from extrapolations of the inverse temperature $\epsilon_{L, \chi}(H)$ where the susceptibility obtains its maximum, see also Fig. 5.4(c). The latter are expected to scale as [129]

$$
\epsilon_{L, \chi}(H)=\epsilon_{\infty}(H)+\frac{a}{\ln (L / b)^{1 / \nu}},
$$

with non-universal constants $a$ and $b$, and $\nu$ characterizing the divergence of the correlation length at a Kosterlitz-Thouless -transition, $\xi \propto \exp \left(a t^{\nu}\right)$ and $t=\epsilon_{\infty}(H)-\epsilon$. However, as $\nu$ is also unknown (we cannot use the $\mathrm{XY}$ value $\nu_{X Y}=1 / 2$ as the critical exponents in two dimensions did not match, see 5.3.2) we are left with a four-parameter fit, and for this reason we consider this method unsuitable.

To summarize the results for $p=8$ we still construct the capillary phase diagram. To this end, we plot the energy densities of the coexisting isotropic $\left(\rho_{\text {iso }}(H)\right)$ and (quasi-)nematic $\left(\rho_{\text {nem }}(H)\right)$ phases as function of inverse film thickness $1 / H$ (these values are simply the peak positions in the energy density distribution, see Fig. 5.10 , and can be directly "read-off"). The extrapolation to the thermodynamic limit of $\rho_{L, \text { iso }}$ and $\rho_{L, \text { nem }}$ obtained in finite systems is assumed to take the form

$$
\rho_{\infty}(H)-\rho_{L}(H) \propto 1 / V .
$$

Of course, this procedure only makes sense when $H>H_{x}$, because the peaks $\rho_{\text {iso }}$ and $\rho_{\text {nem }}$ exist only for first-order and not continuous phase transitions in the thermodynamic limit. Hence, the binodal lines in the phase diagram terminate at $H=H_{x}$. The resulting capillary phase diagram is shown in Fig. 5.8.

\subsubsection{Finite-film thickness: the case $p=20$}

We have seen in the previous section that by increasing the film thickness, the isotropic-nematic transition ultimately becomes first-order, beyond some crossover thickness $H_{x}$. However, for $p=20$, the transition is first-order already for $H=1$, and here the crossover does not occur. Consequently, in the capillary phase diagram, 


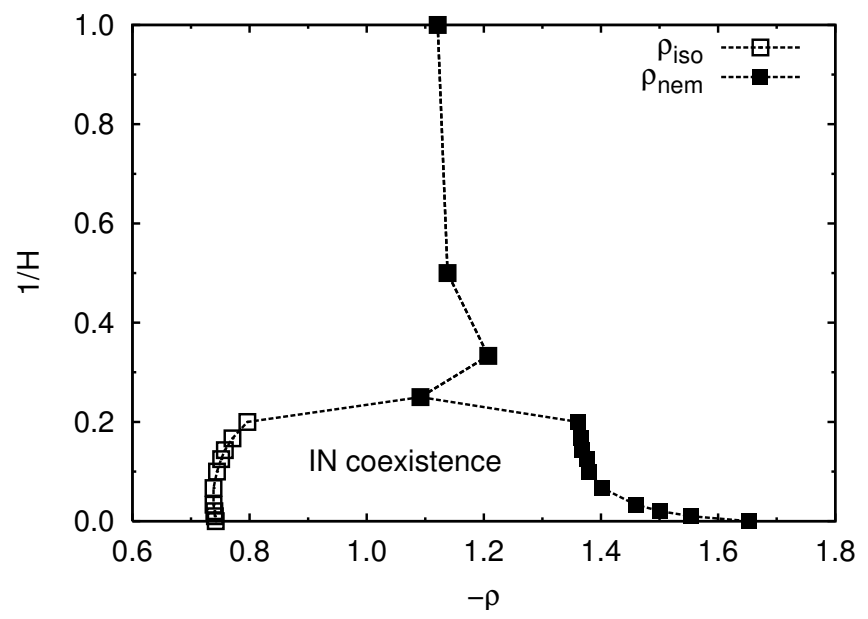

Figure 5.8: Capillary phase diagram with $p=8$. Shown is the variation of the coexisting isotropic energy density $\rho_{\text {iso }}(H)$ and nematic density $\rho_{\text {nem }}(H)$ with inverse film thickness $1 / H$. The phase transition is first-order up to the crossover inverse thickness at $1 / H_{x} \sim 0.33$, where the individual branches terminate. Isotropic-nematic phase coexistence is observed only within the enclosed area.

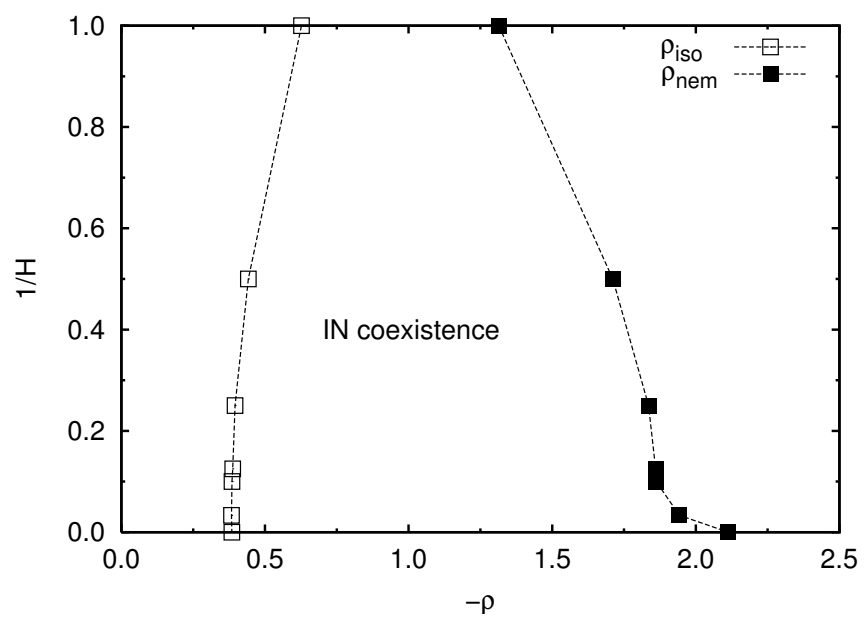

Figure 5.9: Capillary phase diagram with $p=20$. The branches do not terminate at any inverse thickness $1 / H$ but continue to the $2 \mathrm{D}$ limit (corresponding to $1 / H=1)$. Isotropic-nematic coexistence can be observed anywhere between the two branches. 


\section{Confinement of nematic liquid crystals}

the isotropic and nematic branches do not terminate. By using the extrapolation of the energy densities $\rho_{L \text {,iso }}$ and $\rho_{L, \text { nem }}$ as in Eq.(5.7), we can immediately construct the resulting capillary phase diagram, shown in Fig. 5.9. In contrast to the capillary phase diagram for $p=8$ shown in Fig. 5.8, the isotropic and nematic branches do not terminate, and isotropic-nematic phase coexistence can be observed at arbitrary film thickness.

\subsection{Application of the Kelvin equation}

In this section we study the change in transition inverse temperature $\epsilon_{\infty}(H)$ with film thickness $H$, but only for values of $H$ where the corresponding isotropic-nematic transition is first-order. In this case, we expect the Kelvin equation to hold [111]:

$$
\Delta \epsilon \equiv 1-\frac{\epsilon_{\infty}}{\epsilon_{\infty}(H)}=\frac{2 \gamma_{\infty}}{\mathcal{L}_{\infty} H}
$$

where $\Delta \epsilon$ is the (normalized) shift in inverse temperature, $\epsilon_{\infty}$ is the bulk transition inverse temperature, $\gamma_{\infty}$ is the bulk interfacial tension, and $\mathcal{L}_{\infty}$ is the bulk latent heat density. The form of Eq.(5.8) is particularly useful as all quantities appearing can be extracted from finite-size simulation data. At a first-order phase transition, for example, $\epsilon_{\infty}(H)$ can be determined by the finite-size scaling methods of sections 4.4 .2 and 4.4.3. We can calculate the latent heat $\mathcal{L}_{L}(H)$ for a particular system of size $L$ using the corresponding energy probability distribution $\ln P(\rho)$. The distance between the isotropic and nematic peaks, $\Delta \rho \equiv \rho_{\text {nem }}-\rho_{\text {iso }}$ equals the latent heat density, see also Fig. 5.10. A second way to extract the latent heat is via the specific heat maxima and by using the relation [98]

$$
\mathcal{L}_{L}(H)=\sqrt{4 c_{L, \max }(H) / V}
$$

and this we extrapolate to $L \rightarrow \infty$ assuming $\mathcal{L}_{\infty}(H)-\mathcal{L}_{L}(H) \propto 1 / V$, similar to Eq.(5.7).

The bulk surface tension is related to the free energy barrier as [71]

$$
\gamma_{\infty}=\lim _{L \rightarrow \infty} \gamma_{L}, \quad \gamma_{L}=\Delta F /\left(2 L^{2}\right)
$$

where dimensionality $d=3$ is assumed. To obtain $\gamma_{\infty}$ of the thermodynamic limit, we extrapolate in $L$ using

$$
\gamma_{\infty}=\gamma_{L}+a \ln L / L^{2}+b / L^{2}
$$

with constants $a$ and $b$ [71], and where a cubic periodic $L \times L \times L$ simulation box is used. This method yields values of $\gamma_{\infty} \approx 0.08$ for $p=8$ and $\gamma_{\infty} \approx 0.31$ for $p=20$ (in units of $k_{B} T$ per squared lattice spacing). 


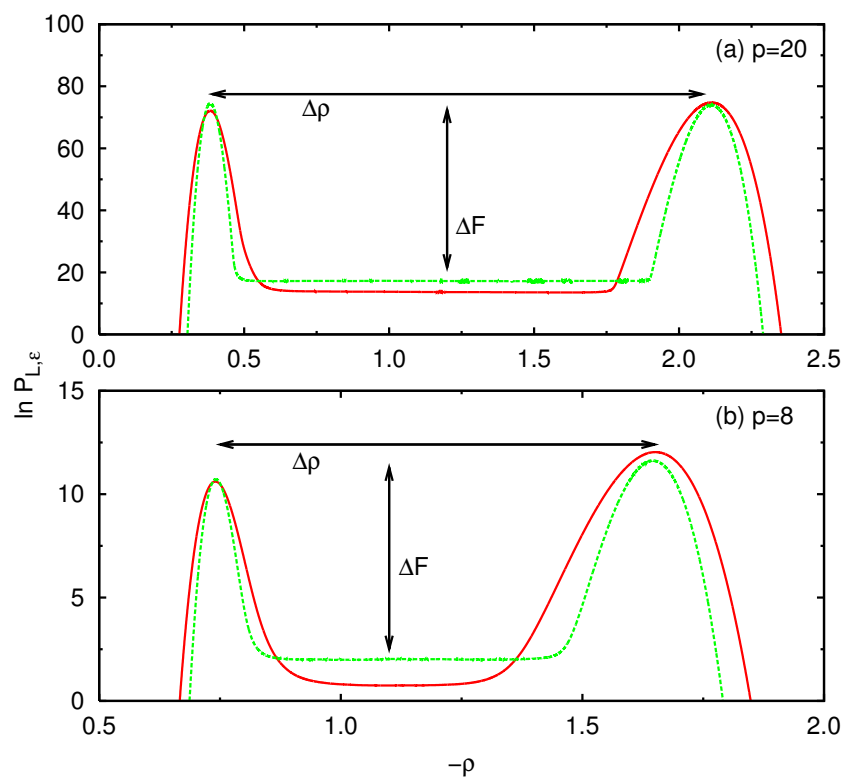

Figure 5.10: Plots of $\ln P(\rho)$ obtained from bulk simulation boxes (periodic boundary conditions in all directions) for system sizes $10 \times 10 \times 30$ (red) and $10 \times 10 \times 60$ (green), for both $p=20$ in (a) and $p=8$ in (b). The height of the peaks to the minimum in-between is related to the interfacial tension $\gamma_{\infty}$ by Eq. 5.10). Note that there is larger discrepancy in $\Delta F$ barriers between the system sizes for $p=8$ than for $p=20$. 
5 Confinement of nematic liquid crystals

Table 5.2: Dependence of the transition inverse temperature $\epsilon_{\infty}(H)$ on the film thickness $H$ for those cases where the isotropic-nematic transition is firstorder, i.e. $H \geq H_{x}$. Results are listed for $p=8$ and $p=20$. As the film thickness increases, the transition inverse temperature approaches the one of the bulk system, i.e. $\epsilon_{\infty}(H) \rightarrow \epsilon_{\infty}$. For completeness, the bulk latent heat density $\mathcal{L}_{\infty}$ and the bulk interfacial tension $\gamma_{\infty}$ are also included, as they are required for the comparison to the Kelvin equation (Eq.55.8).

\begin{tabular}{|c|c|c|c|c|c|}
\hline$p=8$ & $H$ & $\epsilon_{\infty}(H)$ & $p=20$ & $H$ & $\epsilon_{\infty}(H)$ \\
\hline & 5 & 1.614 & & 1 & 2.769 \\
\hline & 6 & 1.593 & & 2 & 2.175 \\
\hline & 7 & 1.578 & & 4 & 1.962 \\
\hline & 8 & 1.568 & & 8 & 1.874 \\
\hline & 10 & 1.555 & & 10 & 1.858 \\
\hline & 15 & 1.540 & & 30 & 1.821 \\
\hline & 30 & 1.528 & & & \\
\hline & 50 & 1.525 & & & \\
\hline & 100 & 1.522 & & & \\
\hline & $\epsilon_{\infty}$ & 1.521 & & $\epsilon_{\infty}$ & 1.806 \\
\hline & $\mathcal{L}_{\infty}$ & 0.909 & & $\mathcal{L}_{\infty}$ & 1.727 \\
\hline & $\gamma_{\infty}$ & 0.065 & & $\gamma_{\infty}$ & 0.300 \\
\hline
\end{tabular}




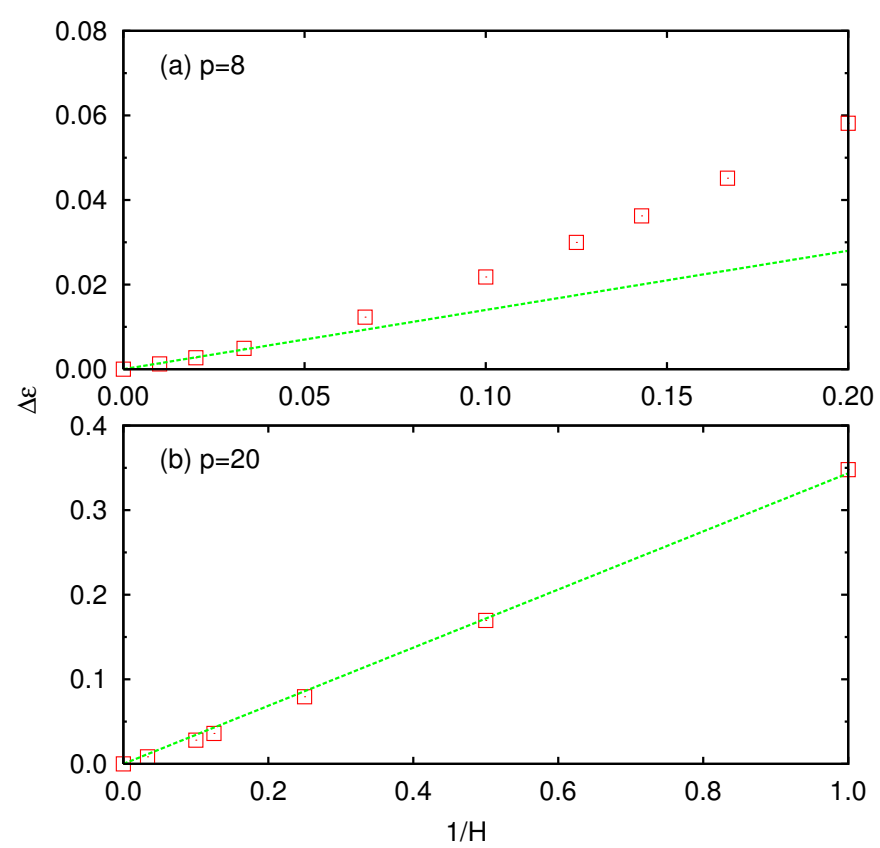

Figure 5.11: The Kelvin equation, Eq. (5.8), tested for (a) $p=8$ and (b) $p=20$. The fits of the inverse temperature shift $\Delta \epsilon$ to inverse film thickness $1 / H$ yield slopes of $a \approx 0.14$ for $p=8$ (when fitting to large $H$ only) and $a \approx 0.34$ for $p=20$.

Rather than using Eq.(5.11), a method to circumvent the finite-size scaling is to use a stretched simulation box of form $L \times L \times D$, with an elongated axis $D \gg L$, but still with periodic boundary conditions [71]. The corresponding energy density probability distributions $\ln P(\rho)$ are shown in Fig. 5.10. We now see a pronounced flat region in the distributions between the peaks. This means that interactions between the interfaces in the coexistence region are small, which in turn implies that $\Delta F$ more accurately reflects the interface free energy. By using this method we obtain $\gamma_{\infty} \approx 0.05$ for $p=8$ and $\gamma_{\infty} \approx 0.29$ for $p=20$. For large $L$ the calculated value of $\Delta F$ should be independent of the elongated axis $D$, but for both $p=20$ in Fig. 5.10 (a) and $p=8$ in (b) we see this is not completely true, especially for $p=8$ (some interaction appears to remain between the interfaces). The estimates of $\gamma_{\infty}$ from the two methods for $p=20$ match well, but for $p=8$ there is small discrepancy; the values quoted in Table 5.2 are the averages of the two methods.

All quantities needed for Eq.(5.8) are now to hand. In Fig. 5.11 we plot the inverse temperature shift $\Delta \epsilon$ against $1 / H$ for the first-order phase transitions of both $p=8$ and $p=20$. If the Kelvin equation holds a linear relation should result. For $p=20$ 
this is clearly the case. According to Eq.(5.8), the slope of a linear fit through this data should equal $a=2 \gamma_{\infty} / \mathcal{L}_{\infty}$. From fitting we find $a \approx 0.34$, whereas using our independent estimates we calculate $2 \gamma_{\infty} / \mathcal{L}_{\infty}=0.35$. The agreement is quite remarkable. For $p=8$, however, the linear fit works only in the limit $1 / H \rightarrow 0$, and therefore it appears that only strongly first-order phase transitions scale according to the Kelvin equation. If we use only the largest system thicknesses, i.e. the systems with the strongest first-order phase transitions, we obtain $a \approx 0.14$ from fitting and $2 \gamma_{\infty} / \mathcal{L}_{\infty}=0.13$ by using the values of Table 5.2 . The agreement is again remarkable.

It has been noted that interfacial fluctuations in complete wetting films give rise to corrections to the Kelvin equation [130]. In this case the $1 / H$ dependency, as seen in Fig. 5.11, is replaced by $1 / H-b l$, where $l$ is the thickness of the wetting film and $b$ is an amplitude constant. However, using this method on the $p=8$ data gives an unphysical wetting film thickness. As a result in Fig. 5.11 we plot $\Delta \epsilon$ against $1 / H$.

\subsection{Summary of simulations between flat walls}

We have found that there is no universal scenario describing the nature of the isotropic-nematic phase transition in thin films. By changing a single parameter governing the sharpness of the pair-interaction of neighboring particles in the generalized Lebwohl-Lasher model, we have been able to bring about first-order phase transitions, continuous phase transitions, and also the absence of transitions. It is generally found that upon decreasing the film thickness, the transition inverse temperatures increases. For strongly first-order phase transitions we find very good agreement to the Kelvin equation. We observe not only the " $1 / H$ " shift of the transition inverse temperature, but by using the independently measured bulk latent heats and interfacial tensions, we also recover the prefactor of this shift. However, when the phase transitions are only weakly first-order, deviations from the Kelvin equation become apparent.

Two different phase diagram topologies were also obtained: the binodals either terminate at some film thickness $H=H_{x}$, or they persist all the way down to the pure $2 \mathrm{D}$ limit. It is interesting that the topology of the $p=8$ diagram (terminating isotropic and nematic branches of the binodal) is also observed in simulations of colloidal rods and plates in confinement [116, 117, 118, 131]. The topology of the $p=20$ phase diagram compares well to some of the experiments [29, 30, 31]. As the binodal branches do not terminate in this case, first-order isotropic-nematic phase transitions are possible in thin-films even in the exact $2 \mathrm{D}$ limit! 


\section{Random quenched disorder in liquid crystals}

\section{Contents}

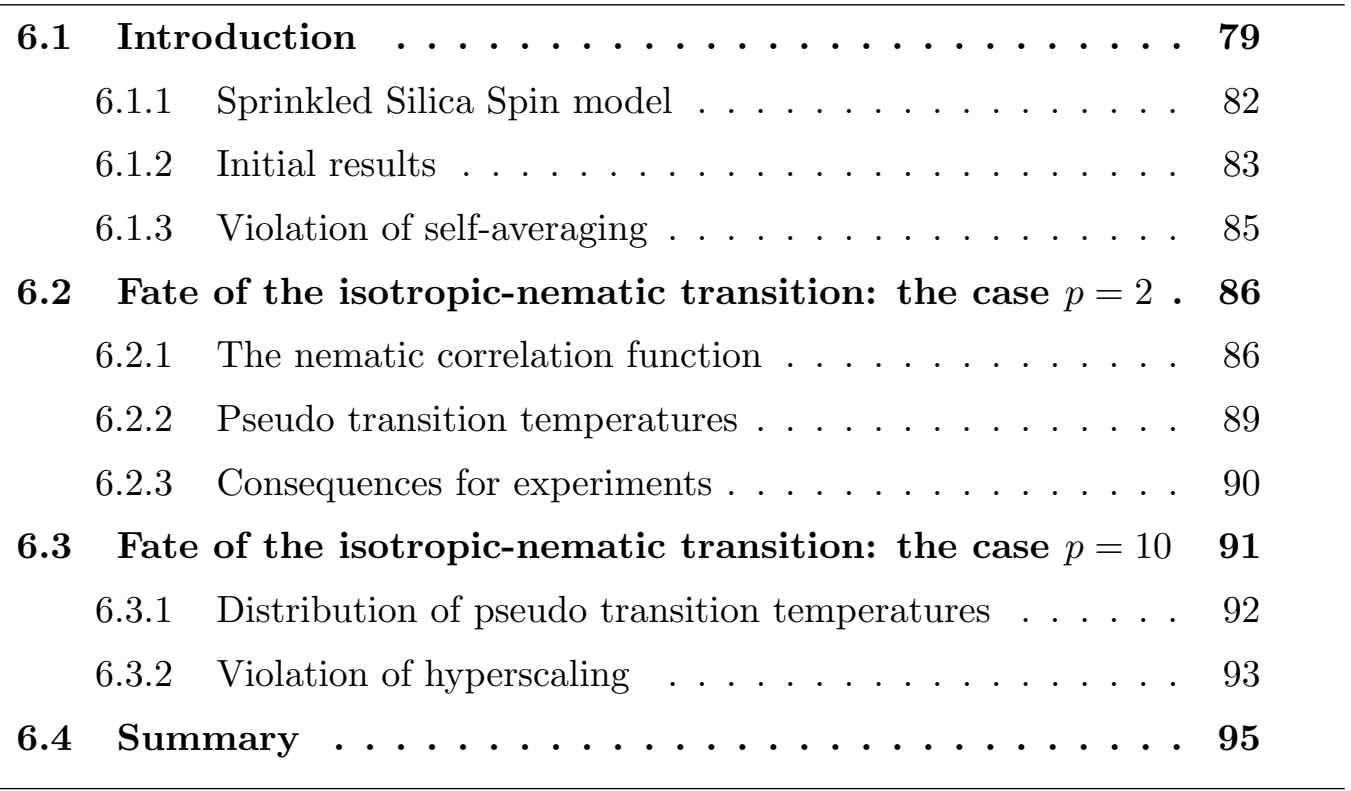

\subsection{Introduction}

The effect of disordered media upon liquid crystal phase transitions has been studied experimentally using various systems [11]. In certain cases, the disordered medium induces a quenched random field. The interaction between the quenched random field and the liquid crystal imposes preferred orientations for the liquid crystal particles at certain (random) locations, see the sketches in Fig. 6.1. The material most commonly used for studying such effects is silica aerogel [132, 133, 134, 135, 136], although certain glasses, such as Vycor have also been used [137, 138]. These porous media are quite remarkable in the sense that they exist almost of "empty space", as can be seen in Fig. 6.2. The volume fraction of aerogel, for example, can be as low as $1 \%$ [139].

A nematic phase in bulk, i.e. in three-dimensions (3D) and in the absence of any external fields or confining walls, exhibits long-range nematic order (LRO). With an 
6 Random quenched disorder in liquid crystals

(a)

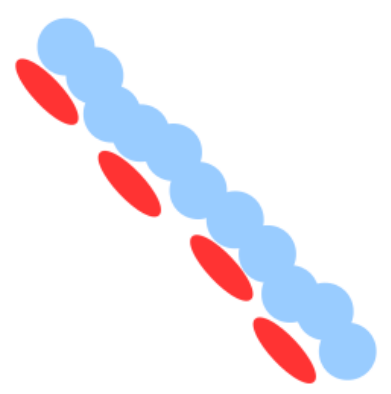

(b)

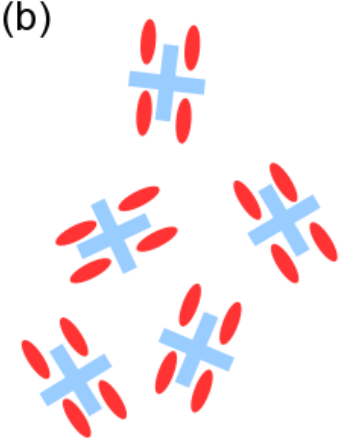

Figure 6.1: (a) Microscopic and (b) macroscopic sketches of how ellipsoidal liquid crystal molecules are anchored to aerogels. In the microscopic view, liquid crystal molecules (red) preferentially align planar to the aerogel branch (blue). In the macroscopic view one observes randomly located and oriented pinning sites, around which liquid crystal molecules align in one direction preferentially.

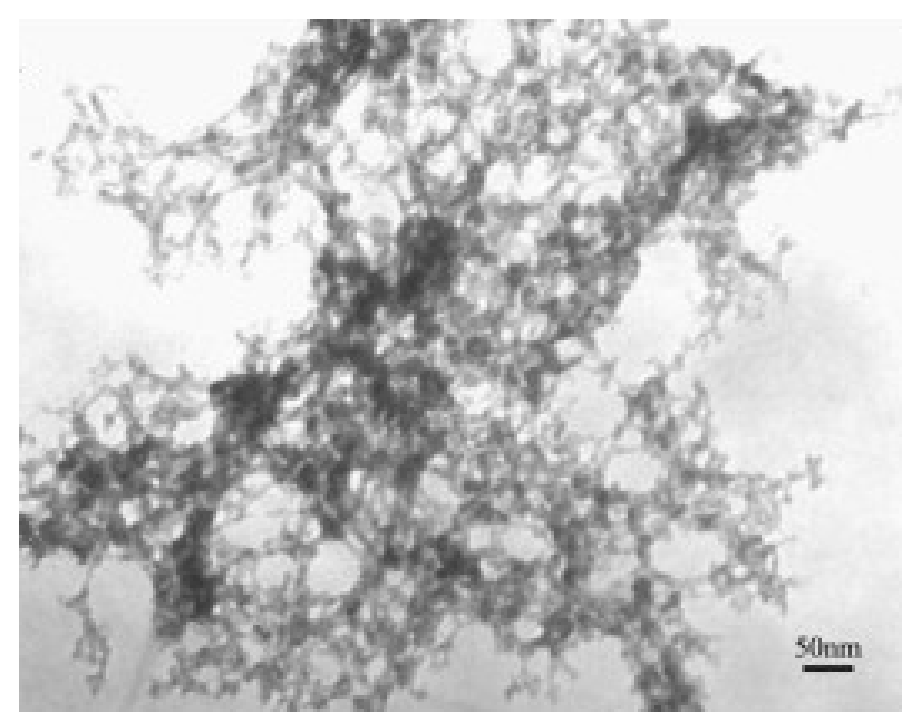

Figure 6.2: Transmission electron microscopy (TEM) micrographs of a silica aerogel, showing three-dimensional net structure and pores $\sim 50 \mathrm{~nm}$. If liquid crystal molecules are inserted into the aerogel, the structure imposes a preferred orientations in random locations. The aerogel has porosity $\sim 95-97 \%$ and is thus fairly transparent. Reprinted from Ref. [139] with permission from Elsevier. 
6.1 Introduction

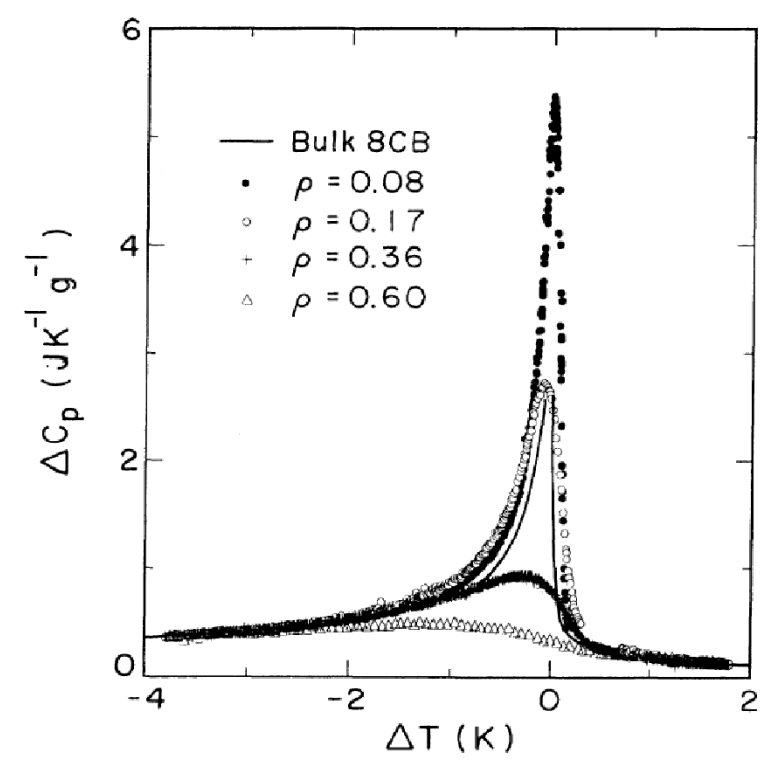

Figure 6.3: Experimental results of the excess heat capacity $\Delta C_{p}(N-I)$ of the isotropic-nematic phase transition for $8 \mathrm{CB}$ in bulk and in four aerogels of densities $\rho=0.08,0.17,0.36,0.60 \mathrm{~g} / \mathrm{cm}^{3}$. The size of the specific heat peak is reduced as aerogel density is increased. Reprinted figure with permission from Ref. [132].

applied quenched random field, LRO is destroyed at finite temperatures in dimensions $d \leq 4$ [140], which thus includes the experimentally relevant cases $d=2,3$. However, just because LRO is destroyed does not mean that phase transitions are not possible to realize. In fact, isotropic-nematic phase transitions in the presence of external fields are often observed in experiments [132, 134, 136, 141].

The isotropic-nematic phase transition in bulk is usually first-order. It has been predicted that random fields can change the order of the isotropic-nematic phase transition, either making it continuous, or even destroying it completely [142]. Experiments on liquid crystals also show similar effects. For example, the liquid crystal $8 \mathrm{CB}$ undergoes a weakly first-order isotropic-nematic phase transition in bulk at $\sim 313.2 \mathrm{~K}[28]$. When $8 \mathrm{CB}$ is confined in the connected porous network of a silica aerogel the isotropic-nematic phase transition is found to be rounded and the first-order transition is replaced by a continuous phase transition [134, 132]. Furthermore, the phase transition is shifted to occur at a lower temperature. These effects are seen in Fig. 6.3 .

A further important theoretical result of systems in quenched random fields is that they do not necessarily self-average [143, 144, 145]. Normally, if we have two 
6 Random quenched disorder in liquid crystals

very small samples of the same material and we take measurements on each, we likely obtain two different results, due to fluctuations within the samples. However, if we double the size of the samples and take measurements again, we would expect the results to be more similar than for the smaller samples owing to the central limit theorem. We could then repeat this doubling of sample size until we reach a large (macroscopic) sample where the results should be identical. This is the usual scenario. However, if self-averaging is violated this need not be true. Individual samples, even when macroscopically large, will still reveal fluctuations between them. This naturally poses a problem when comparing results between different experiments. For example, the specific heat maximum of a nematic liquid crystal confined within an aerogel may be found at different temperatures for different samples of the aerogel! In the extreme case, in the absence of self-averaging, the distribution of the specific heat maxima may not become sharp in the thermodynamic limit! In this chapter we investigate these subtleties in more detail by means of computer simulation and finite-size scaling.

\subsubsection{Sprinkled Silica Spin model}

We use the Sprinkled Silica Spin model [146] to simulate nematic liquid crystals in disordered media [147, 148, 146, 149, 150]. It is very similar to the Lebwohl-Lasher model, but with the additional ingredient of quenched disorder. The SprinkledSilica-Spin model is defined on a 3D periodic lattice of volume $V=L \times L \times L$ with system side length $L$. A $3 \mathrm{D}$ unit vector $\vec{d}_{i}$ (spin) is attached to each lattice site $i$. The spins interact with their nearest neighbors at sites $j$ via

$$
\mathcal{H}=-\epsilon \sum_{\langle i, j\rangle}\left|\vec{d}_{i} \cdot \vec{d}_{j}\right|^{p}
$$

with coupling constant $\epsilon>0$ and the sum over nearest neighbors denoted by $\langle i, j\rangle$. The factor $1 /\left(k_{B} T\right)$ is incorporated into the coupling constant $\epsilon$, with $k_{B}$ the Boltzmann constant and $T$ the temperature; the parameter $\epsilon$ is thus a measure of inverse temperature. Increasing the exponent $p$ has the effect of making the shape of the interaction sharper and narrower, as was explained in section 3.4 .

The difference between the Lebwohl-Lasher model and the Sprinkled-Silica-Spin model is that a fraction $q$ of spins are quenched. These spins are assigned some orientation at the start of the simulation but remain static thereafter. The quenched spins are randomly chosen and randomly oriented (although still being unit vectors, of course). This can be conceived of as a random field of infinite strength acting on a fraction of the spins. It is important for us to choose the fraction of quenched spins carefully. If $q$ is very small we would require enormous system sizes to observe any effect of the quenched spins, whereas if $q$ is too large the non-quenched spins will no longer form a percolating network. In this latter case a true phase transition (i.e. a phase transition in the thermodynamic limit) trivially cannot occur because 
correlations cannot propagate throughout the entire lattice. We use $q=0.10(10 \%$ of all spins) throughout this work. As we shall see, this is sufficient to observe a pronounced random-field effect.

Because the Sprinkled-Silica-Spin model exhibits metastable states [151, 152], it is important to use a simulation method that performs a random walk in phase space. A standard Monte Carlo method, sampling states directly from the Boltzmann distribution, may "get stuck" and therefore we use two broad histogram sampling methods, namely Wang-Landau sampling [67] and successive umbrella sampling [76], see sections 2.4.2 and 2.4.3 respectively. By using these methods we can measure $P_{L, T}^{(k)}(\rho, S)$, the probability distribution of the energy density $\rho$ and the nematic order parameter $S$, at system size $L$ and temperature $T$, for some arrangement of quenched spins $k$. The energy density is defined via the Hamiltonian of Eq. 6.1) as $\rho=\mathcal{H} /\left(\epsilon L^{3}\right)$; the nematic order parameter $S$ was defined in section 1.4 .1 .

\subsubsection{Initial results}

In contrast to previous chapters we must now perform many simulations of the same system size in order to obtain averages over many different realizations of the quenched disorder. The importance of the disorder average is clear from Fig. 6.4(a) where we see results for two randomly chosen realizations of disorder of a $L=10$ system governed by Eq. (6.1) with $p=2$. The maxima of the specific heat $c=$ $L^{3}\left(\left\langle\rho^{2}\right\rangle-\langle\rho\rangle^{2}\right)$ curves occur at different inverse temperatures $\epsilon$ and they are also of different magnitude, a trend that is also observed for the nematic susceptibility $\chi=L^{3}\left(\left\langle S^{2}\right\rangle-\langle S\rangle^{2}\right)$ curves.

Therefore, for each random-field sample $k$ of size $L \times L \times L$, we associate a pseudocritical inverse temperature $\epsilon_{c, k}$, defined as the inverse temperature of the specific heat maximum (alternatively, one could use the temperature of the susceptibility maximum also). We simulate the Sprinkled-Silica-Spin model for $M \approx 1000-2500$ random field samples for each system size, waiting for the "running average" values of quantities of interest, such as the pseudo-critical inverse temperatures, to reach plateau values. In Fig. 6.4(b) we show the average of the inverse temperatures of the specific heat maxima $\left[\epsilon_{c}\right]$, and in Fig. 6.4(c) the corresponding sample-to-sample fluctuation

$$
\delta \epsilon_{c}=\sqrt{\left(\left[\epsilon_{c}^{2}\right]-\left[\epsilon_{c}\right]^{2}\right)},
$$

both with $L=10$ and $p=2$. The square brackets [.] signify the disorder average and is calculated as

$$
\left[\epsilon_{c}^{n}\right]=(1 / M) \sum_{k=1}^{M} \epsilon_{c, k}^{n} .
$$

As shown in Fig. 6.4, both $\left[\epsilon_{c}\right](\mathrm{b})$ and $\delta \epsilon_{c}$ (c) require $M \approx 2000$ realizations before plateau values are reached. 
6 Random quenched disorder in liquid crystals
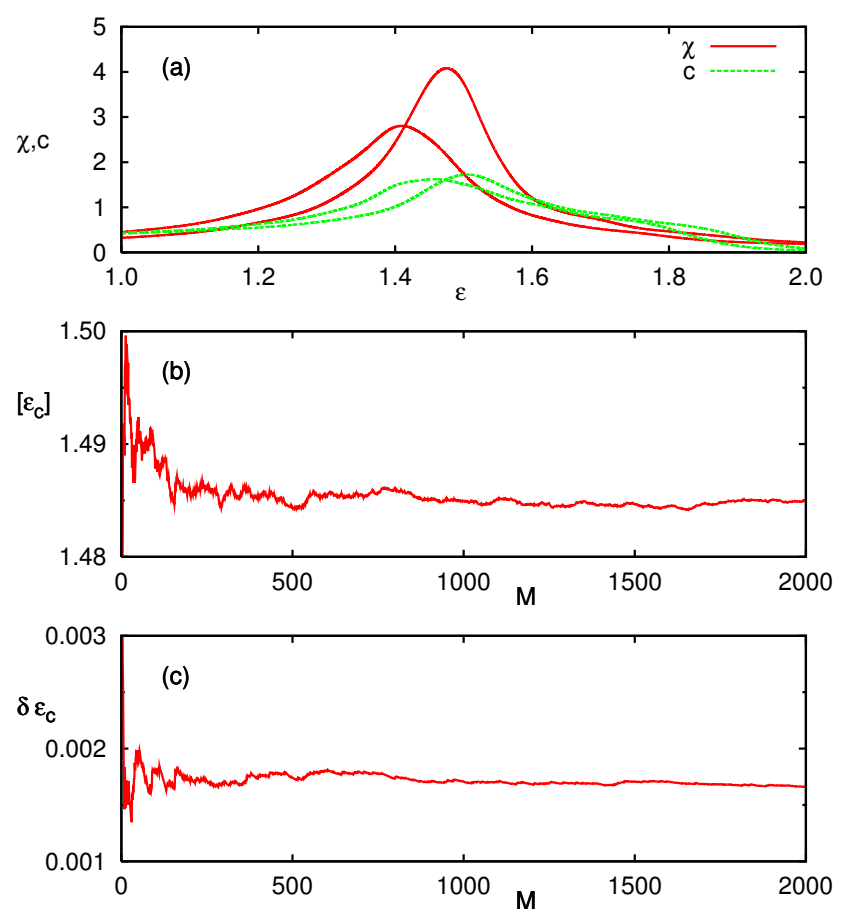

Figure 6.4: (a) Different realizations of disorder give different inverse temperatures $\epsilon$ and heights of the susceptibility $\chi$ and specific heat $c$ peaks. The $\chi$ and $c$ curves with the peaks more toward the left come from one realization of disorder, the curves with the peaks on the right from a second realization of disorder. The number of disorder realizations $M$ needed to obtain accurate results is large, see (b) and (c). It requires $M \approx 2000$ realizations of disorder until the average transition temperature of the specific heat maxima $\left[\epsilon_{c}\right](b)$, and the fluctuations in these temperatures $\delta \epsilon_{c}$ (c) reach plateau values. Data for all three plots is obtained using Eq. 6.1) with $p=2$ from Eq. 6.1) on 3D lattices of side length $L=10$. 
From Fig. 6.4 it clearly follows how important it is to simulate a sufficiently large set of quenched disorder samples, as results between samples differ $\left(\delta \epsilon_{c}>0\right)$. In a pure system (without quenched disorder) there are only thermal, and not disorder, fluctuations. Thermal fluctuations typically become large near phase transitions. For example, the thermal order parameter fluctuations define the susceptibility

$$
\chi=L^{d}\left(\left\langle S^{2}\right\rangle-\langle S\rangle^{2}\right),
$$

which for a system with quenched disorder generalizes to

$$
\chi_{\mathrm{con}}=L^{d}\left[\left\langle S^{2}\right\rangle-\langle S\rangle^{2}\right],
$$

with $\langle\cdot\rangle$ a thermal average and [.] the disorder average (it is convention in the spinglass community to call the above fluctuation the connected susceptibility, hence the subscript "con"). In the presence of quenched disorder, however, we can also define different fluctuations (called disconnected fluctuations) which do not have their analog in pure systems. The role of the disconnected fluctuations in the SprinkledSilica-Spin model will be discussed next.

\subsubsection{Violation of self-averaging}

To this end, we consider the fluctuation in the thermally averaged nematic order parameter $\langle S\rangle$ between quenched disorder samples. For each random-field sample $k$ we measure the thermally averaged nematic order parameter $\langle S\rangle_{k}$ (using WangLandau or successive umbrella sampling sampling) and then calculate the sampleto-sample fluctuation

$$
R_{\mathrm{dis}}^{2}=\left[\langle S\rangle^{2}\right]-[\langle S\rangle]^{2}, \quad\left[\langle S\rangle^{n}\right]=\frac{1}{M} \sum_{k=1}^{M}\langle S\rangle_{k}^{n},
$$

This is called a disconnected fluctuation, and it only has meaning in a system with quenched disorder, because in a pure system the disorder average [.] does not exist and one trivially obtains $R_{\text {dis }}=0$. If the system self-averages $R_{\text {dis }} \rightarrow 0$ in the thermodynamic limit $L \rightarrow \infty$. In this case a single large sample would be representative for all samples. If, however, $R_{\text {dis }}$ remains finite in the thermodynamic limit, then self-averaging is violated and a single sample will not be representative for all samples (even if the sample were infinitely large).

In Fig. 6.5 we plot $R_{\text {dis }}$ against "inverse temperature" $\epsilon$ for three different system sizes. The data show that $R_{\text {dis }}$ decreases with increasing system size for small$\epsilon$, and hence the system is self-averaging in this regime. However, at high- $\epsilon$ (low temperature) $R_{\text {dis }}$ increases with system size, implying that self-averaging is violated here. Note also that the two regimes are separated by a maximum in $R_{\text {dis }}$; the inverse temperature of the maximum will be used to define $\epsilon_{R}$ in what follows; $\epsilon_{R}$ thus denotes the inverse temperature at which self-averaging is maximally violated. Note that $\epsilon_{R}$ is $L$-dependent. 
6 Random quenched disorder in liquid crystals

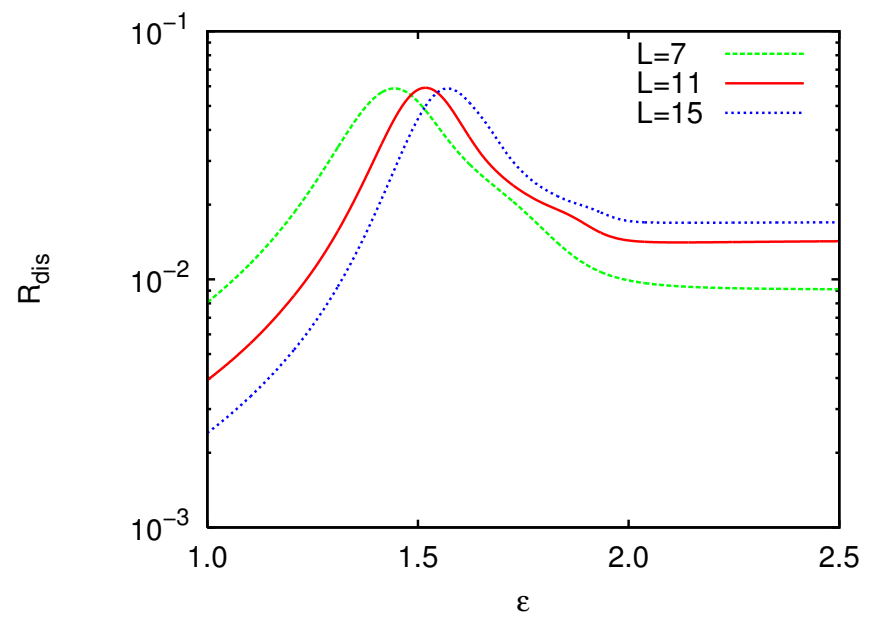

Figure 6.5: $R_{\text {dis }}$ versus $\epsilon$ for $p=2$. The inverse temperature of the $R_{\text {dis }}$ maximum defines $\epsilon_{R}$. At low- $\epsilon$ there is self-averaging $\left(R_{\text {dis }}\right.$ decreases with $\left.L\right)$. However, at high- $\epsilon$ self-averaging is violated $\left(R_{\text {dis }}\right.$ increases with $\left.L\right)$.

\subsection{Fate of the isotropic-nematic transition: the case $p=2$}

In order to see the effect of self-averaging violation on the isotropic-nematic transition we first specialize to using $p=2$ in Eq. 6.1). One mechanism which can induce violation of self-averaging is a large correlation length $\xi$. The Brout argument [153] pictures the thermodynamic limit as being an infinitely large sample consisting of many independent, characteristic subsamples of size $\xi$, as seen in Fig. 6.6. However, this picture breaks down if the correlation length were to become very large. In that case the subsamples will not be independent from each other but "interact".

\subsubsection{The nematic correlation function}

To see if the correlation length is large in the Sprinkled-Silica-Spin model (with $p=2$ ) we consider the nematic correlation function

$$
G_{k}(r)=\left\langle\frac{3}{2}(\vec{d}(0) \cdot \vec{d}(r))^{2}-\frac{1}{2}\right\rangle
$$

In this work we calculate $G_{k}(r)$ using all spins, both free and quenched. The function $G_{k}(r)$ measures the thermally averaged correlation between two spins at distance $r$ apart for some sample of quenched disorder $k$. In a periodic box of side length $L$ we can compute the correlation function up to distances $r=L / 2$. The value at the maximum distance is related to the thermal average of the nematic order 
6.2 Fate of the isotropic-nematic transition: the case $p=2$

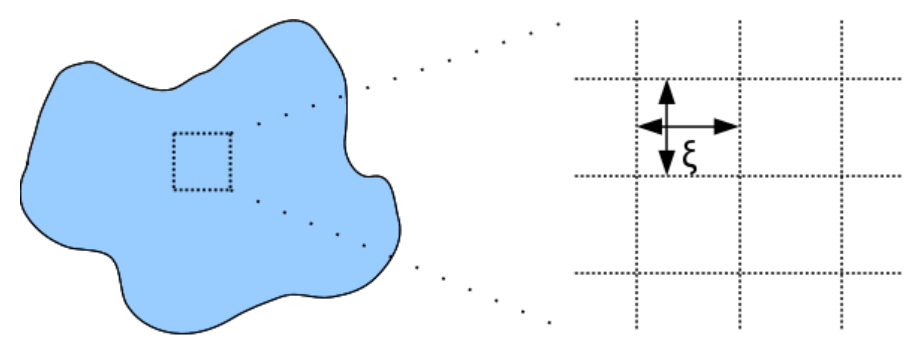

Figure 6.6: A visual representation of the Brout argument [153]. When selfaveraging applies, a large sample (left) can be subdivided into a "grid" of many statistically identical and independent subsamples (right), each of size $\xi$. However, if the the correlation length becomes very large a the division into independent subsamples is no longer possible, and selfaveraging is no longer guaranteed.

parameter in that sample as $G_{k}(L / 2)=\langle s\rangle_{k}^{2}$. From our initial measurements of $R_{\text {dis }}$ in Fig. 6.5 we already concluded that $\langle s\rangle_{k}$ varies between samples, which implies that also $G_{k}(r)$ will reveal sample-to-sample fluctuations.

In Fig. 6.7(a) we show $G_{k}(r)$ at $\epsilon=2$ for a number of quenched disorder samples. This value of $\epsilon$ is significantly above the value where the specific heat and susceptibility maximum occur, and thus we are deep in the "nematic" phase where the system is non-self-averaging, see Fig. 6.5. We indeed observe substantial fluctuations in the correlation function between samples. Also shown in Fig. 6.5(a) is the disorder averaged correlation function $[G(r)]$ calculated using

$$
\left[G(r)^{n}\right]=\frac{1}{M} \sum_{k=1}^{M} G_{k}^{n}(r) .
$$

Following the Imry-Ma argument [140], for a continuous spin system in $d \leq 4$ dimensions we expect the complete destruction of long-range nematic order in the thermodynamic limit for any finite amount of quenched (random field) disorder $q>0$. This prediction is indeed confirmed by experimental $G(r)$ measurements using filled nematics [147]. Furthermore, it is consistent with our measurement of $[G(r)]$ which clearly shows a decay with increasing distance. Over the range of $r$ accessible, the decay conforms best to a power-law, in agreement with Ref. [154]. This indicates that the nematic order is quasi-long-ranged.

To find out more about the sample-to-sample fluctuations in the correlation function we next consider

$$
\kappa(r)=\left[G(r)^{2}\right] /[G(r)]^{2},
$$

which may be regarded as the disconnected correlation function [155, 156]. In a pure system this is trivially $\kappa=1$ but in the presence of quenched disorder $\kappa>1$ is also possible. The result is shown in Fig. 6.7(b) for the range $5 \leq r \leq 15$. At 
6 Random quenched disorder in liquid crystals
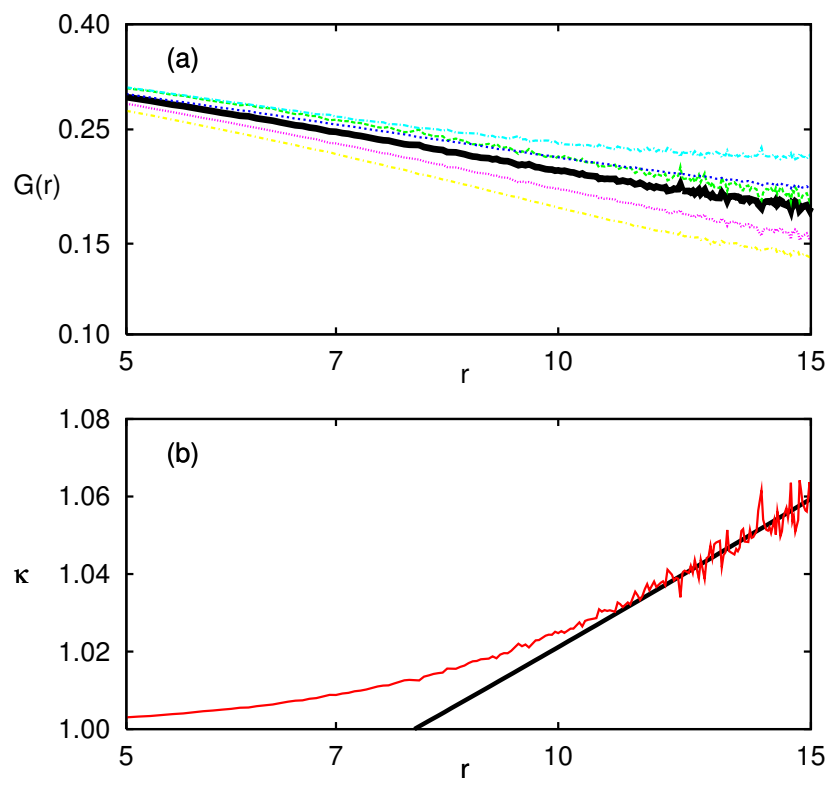

Figure 6.7: The behavior of the connected and disconnected pair correlation functions for the Sprinkled-Silica-Spin model with $p=2, L=30$, and $\epsilon=2.0$. (a) On double-logarithmic scales, we show $G_{k}(r)$ for five samples of quenched disorder (dashed curves), and the disorder-averaged result $[G(r)]$ (solid curve), where $M=100$ random-field samples were used. (b) The variation of the disconnected correlation $\kappa(r)$ where importantly $\kappa>1$ for large $r$. The solid curve is a power-law fit to the large- $r$ regime, where the fit $\kappa \propto r^{\theta}$ with $\theta \approx 0.10$. 
6.2 Fate of the isotropic-nematic transition: the case $p=2$
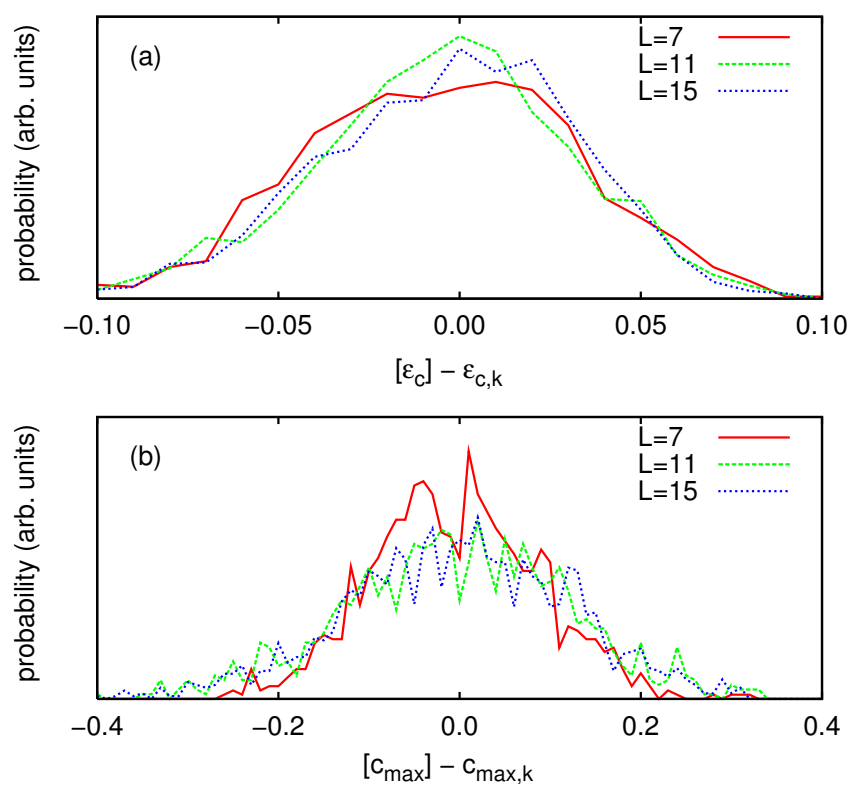

Figure 6.8: Histograms of $\epsilon_{c, k}$ (a) and $c_{\max , k}(\mathrm{~b})$ of the specific heat, shifted by their respective averages, and for several $L$. The histograms do not become sharp as $L$ increases.

small $r$ we observe $\kappa \sim 1$ but for larger values we clearly see an increase. Over the range of $r$ that we can simulate we observe power-law growth $\kappa \propto r^{\theta}$ with $\theta \approx 0.10$. The fact that $\kappa(r)$ increases means that the connected correlations are decaying independently from the disconnected correlations. If the system were self-averaging we would expect both types of correlations to decay similarly, implying $\theta=0$.

\subsubsection{Pseudo transition temperatures}

We now consider the distribution in the pseudo transition temperatures. We compute the inverse temperature $\epsilon_{c, k}$ where the specific heat attains its maximum for each random field sample. In addition we record the corresponding value of the specific heat at the maximum $c_{\max , k}$. In Fig. 6.8(a) we show the histogram of observed $\epsilon_{c, k}$ values, shifted by the average $\left[\epsilon_{c}\right]$, for system sizes $L=7,11,15$. The crucial result is that the histograms do not become sharp as $L$ increases. This implies that the temperature of the isotropic-nematic transition is not well-defined, but rather that each sample of random fields undergoes the transition at a different temperature, even if the sample is large. 
6 Random quenched disorder in liquid crystals

Table 6.1: Properties of systems with $p=2$ and $q=0.1$. Listed are the disorder average inverse temperatures of the specific heat maximum $\left[\epsilon_{c}\right]$, the inverse temperature and temperature of the $R_{\text {dis }}$ maximum $\epsilon_{R}$, and the disorderaveraged specific heat maximum $\left[c_{\max }\right]$ for system sizes $L=7,11,15$.

\begin{tabular}{c|cc|c}
$L$ & {$\left[\epsilon_{c}\right]$} & $\epsilon_{R}$ & {$\left[c_{\max }\right]$} \\
\hline 7 & 1.469 & 1.444 & 1.545 \\
11 & 1.527 & 1.518 & 1.481 \\
15 & 1.557 & 1.571 & 1.382 \\
\hline
\end{tabular}

Similar effects are seen in the values $c_{\max , k}$ of the specific heat at the maxima. The corresponding histograms are shown in Fig. 6.8 (b) shifted by the average $\left[c_{\max }\right]$. Again, we observe that the width of the histograms is independent of system size: the histograms are not becoming narrower and sharper as $L$ increases.

In short, for the Sprinkled-Silica-Spin model with the parameters considered here, the violation of self-averaging is so extreme, that, based on our finite-size scaling analysis, we must conclude that no "true" isotropic-nematic phase transition occurs. While in finite samples we do observe a maximum in the specific heat, the distribution of (pseudo) transition temperatures as well as the value of the specific heat itself, do not become sharp in the thermodynamic limit. In addition we observe that the quenched-averaged maximum value of the specific heat $\left[c_{\max }\right]$ decreases with $L$, see Table 6.1. This furthermore suggests the absence of a true phase transition since otherwise $\left[c_{\max }\right]$ should increase with $L$ [89].

We now discuss the temperature range of the non-self-averaging regime. We notice that $\left[\epsilon_{c}\right]$ is very close to the inverse temperature $\epsilon_{R}$ where $R_{\text {dis }}$ is maximal (see Table 6.1]. As $L$ increases both $\left[\epsilon_{c}\right]$ and $\epsilon_{R}$ increase. The non-self-averaging regime thus becomes smaller in larger systems, i.e. one needs to go to ever lower temperatures to observe it. An interesting question is whether $\epsilon_{R}$ remains finite in the thermodynamic limit. Unfortunately we do not know of a rigorous finitesize-scaling theory describing the shift of $\epsilon_{R}$ with system size. The increase of $\epsilon_{R}$ with $L$ is in any case very slow. For example, if we assume a power-law shift $1 / \epsilon_{R}-1 / \epsilon_{\infty} \propto 1 / L^{y}, 1 / \epsilon_{\infty} \equiv \lim _{L \rightarrow \infty} 1 / \epsilon_{R}$, then in the maximal scenario $\left(1 / \epsilon_{\infty}=0\right)$ we obtain an exponent of at most $y_{\max } \sim 0.16$.

\subsubsection{Consequences for experiments}

Because the decay of $1 / \epsilon_{R}$ and $1 /\left[\epsilon_{c}\right]$ with $L$ are so slow we expect that the non-selfaveraging regime would survive in macroscopic samples. In experiments we would therefore expect that the specific heat maxima will occur at different temperatures, depending on the particular aerogel sample used (even if the samples appear to be identical). In bulk the liquid crystal 8CB undergoes a weakly first-order isotropicnematic phase transition, as does Eq. 6.1) with $p=2$ and $q=0$ (i.e. without 
6.3 Fate of the isotropic-nematic transition: the case $p=10$
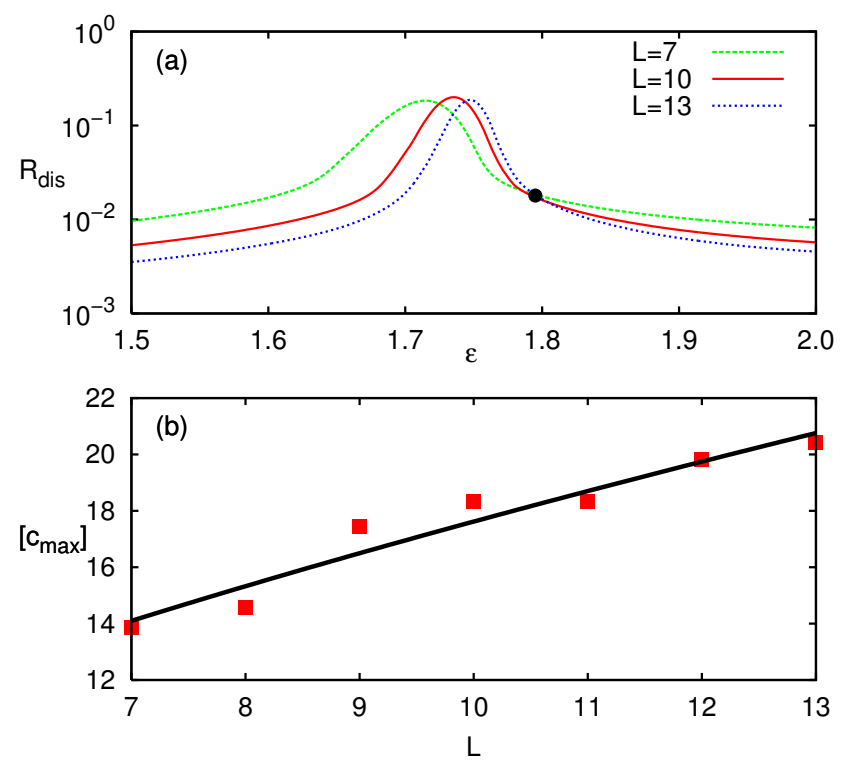

Figure 6.9: (a) $R_{\text {dis }}$ versus $\epsilon$ for $p=10$ for $L=7,10,13$. Self-averaging is observed at both low- and high- $\epsilon$, and there is only a small intermediate region where self-averaging is violated. (b) The quenched-averaged specific heat maxima $\left[c_{\max }\right]$ versus system size $L$ for $L=7-13$. The specific heat maxima diverge $\left[c_{\max }\right] \propto L^{\bar{\alpha}}$ with $\bar{\alpha} \approx 0.62$, with this fit shown by the solid curve, consistent with a continuous transition.

quenched disorder). When inserted in silica aerogel of density $\rho=0.36 \mathrm{~g} / \mathrm{cm}^{3}$ the enthalpy $H$ of the isotropic-nematic phase transition obtained from different samples varies between $3.6-5.23 \mathrm{~J} / \mathrm{g}$ [134, 132], noted as being particularly large [132]. This is of note because the enthalpy is fundamentally linked to the specific heat. Therefore, as we have seen that the specific heat can be non-self-averaging, such a large fluctuation in the enthalpy could be consistent with our observation of nonself-averaging behavior.

\subsection{Fate of the isotropic-nematic transition: the case $p=10$}

We have seen that the Sprinkled-Silica-Spin model with $p=2$ and $q=0.1$ does not undergo a phase transition. However, an important conclusion of chapter 5 was that the phase behavior of liquid crystals is not determined by a particular universality class, but instead by microscopic details, i.e. the shape of the particle-particle pair interaction. Therefore, we now consider the Sprinkled-Silica-Spin model with $p=10$ in Eq.6.1], but still using a fraction of quenched spins $q=0.1$. 
6 Random quenched disorder in liquid crystals

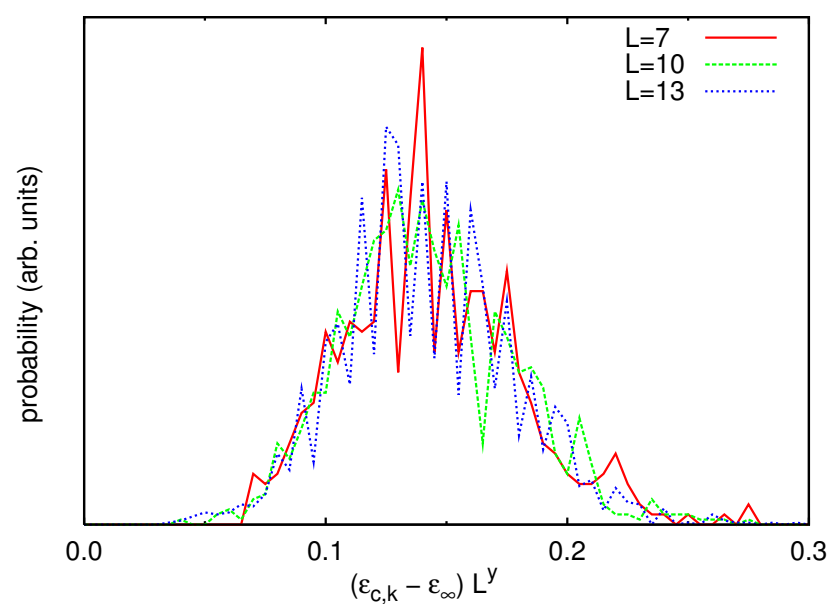

Figure 6.10: Histograms $H\left(\left(\epsilon_{c, k}-\epsilon_{\infty}\right) L^{y}\right)$ obtained using $p=10$ in Eq. 6.1). The histograms for different $L$ collapse, consistent with Eq.6.10 and Eq. 6.11), indicating that a phase transition occurs. The fit parameters used are $\epsilon_{\infty}=1.80$ and $y=0.88$.

Indeed, we observe a remarkable departure from the case $p=2$. In Fig. 6.9.(a) with $p=10$ we now see that self-averaging is conserved at both low- and high- $\epsilon$, and that there is only a small region where self-averaging is violated. This region becomes smaller as $L \rightarrow \infty$, resulting in a critical point at the transition temperature of the thermodynamic limit (marked with the dot in Fig. 6.9(a)).

We believe that the corresponding transition is a genuine one and that it is continuous, based on the variation of the quenched-averaged specific heat maximum $\left[c_{\max }\right]$ with system size $L$, see Fig. 6.9 (b). The fact that $\left[c_{\max }\right]$ increases with $L$ is consistent with the presence of a transition (in contrast to the case $p=2$ where $\left[c_{\max }\right]$ decreases with $L$, see Table 6.1). At a first-order transition it holds that $\left[c_{\max }\right] \propto L^{\bar{\alpha}}$ with $\bar{\alpha}=d=3$. With $p=10$ and $q=0.1$ we instead measure $\bar{\alpha} \approx 0.62$, shown by the solid curve in Fig. 6.9(b), from which we conclude that the transition is continuous.

\subsubsection{Distribution of pseudo transition temperatures}

Next we consider the distribution in the pseudo transition temperatures $\epsilon_{c, k}$ of the specific heat maximum. Since we now observe a transition, we would expect the corresponding histogram to become sharp as $L$ increases (in contrast to Fig. 6.8 for the case $p=2$ ). In fact, we expect the usual scaling predictions to apply [143, 144, 145, 157]. That is, the shift of the quenched-averaged transition temperature 
Table 6.2: Properties of systems with $p=10$ and $q=0.1$. Listed are the disorder average inverse temperatures of the specific heat maximum $\left[\epsilon_{c}\right]$, the inverse temperature and temperature of the $R_{\text {dis }}$ maximum $\epsilon_{R}$, and the disorder average of the specific heat maximum $\left[c_{\max }\right]$ for system sizes $L=7,10,13$.

\begin{tabular}{c|cc|c}
$L$ & {$\left[\epsilon_{c}\right]$} & $\epsilon_{R}$ & {$\left[c_{\max }\right]$} \\
\hline 7 & 1.717 & 1.714 & 13.85 \\
10 & 1.739 & 1.735 & 18.32 \\
13 & 1.751 & 1.747 & 20.42 \\
\hline
\end{tabular}

$\left[\epsilon_{c}\right]=(1 / M) \sum_{k=1}^{M} \epsilon_{c, k}$ is expected to obey

$$
\left[\epsilon_{c}\right]-\epsilon_{\infty} \propto \frac{1}{L^{y}}
$$

with exponent $y>0$, and $\epsilon_{\infty}$ the inverse transition temperature in the thermodynamic limit. Furthermore, the fluctuation in the pseudo transition temperatures $\delta \epsilon_{c}$ defined in Eq. (6.2) is expected to scale similarly

$$
\delta \epsilon_{c} \propto \frac{1}{L^{y}}
$$

Therefore, since both the shift $\left[\epsilon_{c}\right]-\epsilon_{\infty}$ and the fluctuation $\delta \epsilon_{c}$ scale with the same power-law in $L$, we expect the histogram of $H\left(\left(\epsilon_{c, k}-\epsilon_{\infty}\right) L^{y}\right)$ to be $L$-independent, provided that suitable values for $\epsilon_{\infty}$ and $y$ are used. Indeed, the scaling is confirmed in Fig. 6.10 using $\epsilon_{\infty} \approx 1.80$ and $y \approx 0.88$, and the collapse of the histograms for different $L$ is clearly excellent. Note that the value of $\epsilon_{\infty}$ obtained matches the inverse temperature of the intersection of $R_{\text {dis }}$ curves for different $L$ in Fig. 6.9. (a), showing that this is an alternative method for obtaining $\epsilon_{\infty}$. For completeness the numerical data of $\left[\epsilon_{c}\right], \epsilon_{R}$, and $\left[c_{\max }\right]$ for the $L=7,10,13$ systems are also given in Table 6.2.

\subsubsection{Violation of hyperscaling}

We have seen that, unlike pure systems, disordered systems have not only thermal fluctuations but also disorder (sample-to-sample) fluctuations. However, we have not yet directly contrasted these fluctuations. We have already defined the connected susceptibility $\chi_{\text {con }}$ as the thermal order parameter fluctuations averaged over all realizations (see Eq.6.5) ). At a continuous transition the susceptibility diverges with system size $L$ as $\chi_{\text {con }} \propto L^{\bar{\gamma}}$ with critical exponent $\bar{\gamma}$. Note that this scaling-law holds for both conventional and Kosterlitz-Thouless critical points; in case of the 
6 Random quenched disorder in liquid crystals

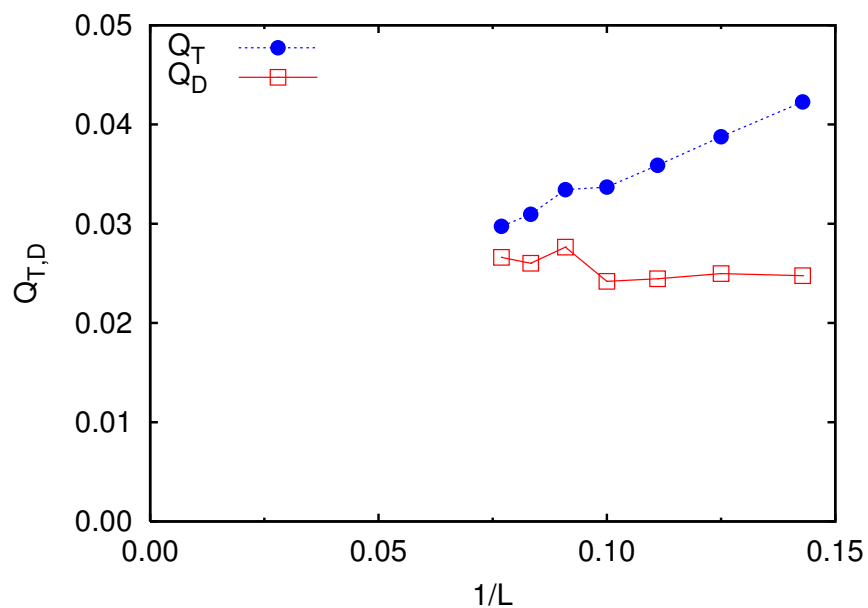

Figure 6.11: The "thermal" cumulant $Q_{T}$ and "disorder" cumulant $Q_{D}$ obtained at the specific heat maximum as function of $1 / L$. The key message to take from this figure is that $Q_{T} \rightarrow 0$ as $L$ increases, while $Q_{D}$ remains constant. This shows that conventional hyperscaling is violated (data obtained for $p=10, q=0.1$, and system sizes $L=7-13)$.

former $\bar{\gamma}$ can still be related to the critical exponent of the correlation length [128]. The equivalent measure for sample-to-sample fluctuations is the disconnected susceptibility

$$
\chi_{\mathrm{dis}}=L^{d}\left(\left[\langle S\rangle^{2}\right]-[\langle S\rangle]^{2}\right),
$$

which scales $\chi_{\text {dis }} \propto L^{\bar{\gamma}_{\text {dis }}}$ at the transition [155]. In order to compare the importance of thermal and sample-to-sample (disorder) fluctuations, we introduce the cumulant ratios $Q_{T}$ and $Q_{D}$

$$
Q_{T} \equiv \frac{\sqrt{\left[\left\langle S^{2}\right\rangle-\langle S\rangle^{2}\right]}}{[\langle S\rangle]}=\frac{\sqrt{\chi_{\mathrm{con}} / V}}{[\langle S\rangle]}
$$

and

$$
Q_{D} \equiv \frac{\sqrt{\left[\langle S\rangle^{2}\right]-[\langle S\rangle]^{2}}}{[\langle S\rangle]}=\frac{\sqrt{\chi_{\mathrm{dis}} / V}}{[\langle S\rangle]},
$$

which, respectively, compare the magnitude of thermal and disorder fluctuations to the order parameter. At a continuous transition the latter is expected to decay as $[\langle S\rangle] \propto L^{-\bar{\beta}}$, defining another exponent $\bar{\beta}$.

To make progress we now derive how $Q_{T}$ and $Q_{D}$ are expected to scale with system size $L$ at the transition. At conventional continuous transitions, meaning without quenched disorder, one typically observes a hyperscaling relation between 
the critical exponents of the form 92 ]

$$
\bar{\gamma}+2 \bar{\beta}=d
$$

here with $d=3$ spatial dimensions. Using the above scaling relations for $\chi_{\text {con }}$ and $[\langle S\rangle]$ one then easily derives that $Q_{T}$ becomes $L$-independent, which is commonly used to locate such transitions (the famous "Binder cumulant intersection method" [92]). The fact that $Q_{T}$ remains finite simply means that the thermal fluctuations of the order parameter remain comparable to the order parameter itself. Thus, in the absence of quenched disorder, $Q_{T}$ is constant and independent of system size at the transition. In Fig. 6.11, where we have plotted the values of $Q_{T}$ (taken at the specific heat maxima), this is not what we observe but instead $Q_{T} \rightarrow 0$ as $L \rightarrow \infty$. The thermal fluctuations thus become negligible as $L$ increases, indicating that hyperscaling is violated in this instance.

In fact it has already been predicted that hyperscaling is often violated in the presence of quenched random fields [158, 159, 160]. Instead the hyperscaling relation of Eq.6.15) should be replaced with a "modified hyperscaling" relation

$$
\bar{\gamma}_{\text {dis }}+2 \bar{\beta}=d,
$$

with $\bar{\gamma}_{\text {dis }}$ the critical exponent of the disconnected susceptibility. If one now uses this modified relation to compute the $L$-dependence of $Q_{T}$ and $Q_{D}$ at the transition, one finds that $Q_{T} \rightarrow 0$ as $L$ increases, while now $Q_{D}$ remains constant (which was our motivation to introduce $Q_{D}$ of Eq.(6.14) in the first place). True to prediction in Fig. 6.11, we see that $Q_{D}$ calculated at the specific heat maxima stays approximately constant with system size. In addition, directly measuring at $\epsilon=\epsilon_{\infty}=1.80$ the values of the critical exponents (using the corresponding scaling laws) we obtain $\bar{\gamma}+2 \bar{\beta}=1.50-1.98$ and $\bar{\gamma}_{\text {dis }}+2 \bar{\beta}=2.80-3.48 \sim 3$, giving further evidence that "standard hyperscaling" is violated, but that the modified relation holds. The fact that $Q_{D}$ remains constant at the transition is extremely important for simulations. Therefore, in finite systems there will be very large fluctuations between disorder samples, and meaningful results thus require a substantial disorder average (as was done for the first time in this thesis).

\subsection{Summary}

In this chapter we have shown that random-field quenched disorder strongly affects the isotropic-nematic phase transition. We found large sample-to-sample fluctuations, a change in the transition type (from first-order to continuous), and violation of self-averaging. For computer simulations it is essential to measure a disorder average comprised of many random-field realizations to gain any meaningful information at all.

For a system that undergoes a weakly first-order phase transition in bulk the violation of self-averaging is so severe that no phase transition occurs at all in the 
6 Random quenched disorder in liquid crystals

thermodynamic limit. For a system that undergoes a more strongly first-order phase transition in bulk the violation of self-averaging is restricted to a single-temperature in the thermodynamic limit. In this case a phase transition does occur but it has rather unusual properties. In particular we find that hyperscaling is violated and replaced by a modified relation. 


\section{Bibliography}

[1] F. Reinitzer, Beiträge zur Kenntniss des Cholesterins, Monatshefte für Chemie / Chemical Monthly 9, 421 (1888).

[2] J. L. Fergason, Liquid Crystals, Scient. Am. 77 (1964).

[3] V. I. Kopp, B. Fan, H. K. M. Vithana, and A. Z. Genack, Low-threshold lasing at the edge of a photonic stop band in cholesteric liquid crystals, Opt. Lett. 23, 1707 (1998).

[4] C. T. Kresge, M. E. Leonowicz, W. J. Roth, J. C. Vartuli, and J. S. Beck, Ordered mesoporous molecular sieves synthesized by a liquid-crystal template mechanism, Nature 359, 710 (1992).

[5] J. S. Beck, J. C. Vartuli, W. J. Roth, M. E. Leonowicz, C. T. Kresge, K. D. Schmitt, C. T. W. Chu, D. H. Olson, and E. W. Sheppard, A new family of mesoporous molecular sieves prepared with liquid crystal templates, Journal of the American Chemical Society 114, 10834 (1992).

[6] G. S. Attard, J. C. Glyde, and C. G. Goltner, Liquid-crystalline phases as templates for the synthesis of mesoporous silica, Nature 378, 366 (1995).

[7] T. Tsutsui, C. P. Lin, S. Saito, S. H. Chen, S. H., and M. J. C., Use of Glass-Forming Liquid Crystal Materials for Electroluminescent Diodes, vol. 425, 225-232 (1996).

[8] P. de Gennes, The Physics of Liquid Crystals (Oxford University Press, Oxford, 1974), first edn.

[9] E. Priestley, P. Wojtowicz, and P. Sheng, Introduction to liquid crystals (Plenum Press, London, 1974).

[10] "The Nobel Prize in Physics 1991", see http://nobelprize.org (31. August 2011).

[11] G. Crawford and S. Žumer, Liquid Crystals in Complex Geometries (Taylor \& Francis, 1996).

[12] J. W. Doane, N. A. Vaz, B.-G. Wu, and S. Žumer, Field controlled light scattering from nematic microdroplets, Applied Physics Lett. 48, 269 (1986). 
Bibliography

[13] J. Fergason, Soc. Inf. Disp., Tech. Dig. 16, 68 (1985).

[14] P. S. Drzaic, Polymer dispersed nematic liquid crystal for large area displays and light valves, Journal of Applied Physics 60, 2142 (1986).

[15] D. Engstrom, R. P. Trivedi, M. Persson, M. Goksor, K. A. Bertness, and I. I. Smalyukh, Three-dimensional imaging of liquid crystal structures and defects by means of holographic manipulation of colloidal nanowires with faceted sidewalls, Soft Matter tbc (2011).

[16] K. Binder, J. Horbach, R. Vink, and A. De Virgiliis, Confinement effects on phase behavior of soft matter systems, Soft Matter 4, 1555 (2008).

[17] R. Vink, Critical behavior of soft matter fluids in bulk and in random porous media: from Ising to random-field Ising universality, Soft Matter 5, 4388 (2009).

[18] B. Jerome, Surface effects and anchoring in liquid crystals, Reports on Progress in Physics 54, 391 (1991).

[19] F. J. Vesely and R. Lukač, How to Simulate Anchoring: Confining the Director, Mol. Cryst. Liq. Cryst. Science and Technology. Section A. Mol. Cryst. Liq. Cryst. 352, 233 (2000).

[20] L. A. Madsen, T. J. Dingemans, M. Nakata, and E. T. Samulski, Thermotropic Biaxial Nematic Liquid Crystals, Phys. Rev. Lett. 92, 145505 (2004).

[21] G. Friedel, Anns. Phys. 18, 273 (1922).

[22] S. D. Hudson, A. J. Lovinger, R. G. Larson, D. D. Davis, R. O. Garay, and K. Fujishiro, Detection of nematic-smectic phase transition in a semiflexible main-chain liquid crystalline polymer, Macromolecules 26, 5643 (1993).

[23] H. Li, X. Zhang, R. Zhang, J. Shen, B. Zhao, and W. Xu, Thermotropic Liquid Crystalline Behavior of an Amphiphilic Polymer Lacking Mesogens, Macromolecules 28, 8178 (1995).

[24] Y. Shao and T. W. Zerda, Phase Transitions of Liquid Crystal PAA in Confined Geometries, J. Phys. Chem. B 102, 3387 (1998).

[25] H. Kelker and B. Scheurle, A Liquid-crystalline (Nematic) Phase with a Particularly Low Solidification Point, Angewandte Chemie International Edition in English 8, 884 (1969).

[26] H. J. Coles and C. Stratielle, Mol. Cryst. Liq. Cryst. 55, 273 (1979). 
[27] P. de Gennes and J. Prost, The Physics of Liquid Crystals (Oxford University Press, Oxford, 1995), second edn.

[28] P. P. Karat and N. V. Madhusudana, Elastic and Optical Properties of Some 4-n-Alkyl-4-Cyanobiphenyls, Mol. Cryst. Liq. Cryst. 36, 51 (1976).

[29] M. M. Wittebrood, D. H. Luijendijk, S. Stallinga, T. Rasing, and I. Muševič, Thickness-dependent phase transition in thin nematic films, Phys. Rev. E 54, 5232 (1996).

[30] D. van Effenterre, R. Ober, M. P. Valignat, and A. M. Cazabat, Binary Separation in Very Thin Nematic Films: Thickness and Phase Coexistence, Phys. Rev. Lett. 87, 125701 (2001).

[31] R. Garcia, E. Subashi, and M. Fukuto, Thin-Thick Coexistence Behavior of 8CB Liquid Crystalline Films on Silicon, Phys. Rev. Lett. 100 (2008).

[32] A. V. Kityk, M. Wolff, K. Knorr, D. Morineau, R. Lefort, and P. Huber, Continuous Paranematic-to-Nematic Ordering Transitions of Liquid Crystals in Tubular Silica Nanochannels, Phys. Rev. Lett. 101, 187801 (2008).

[33] D. Davidov, C. R. Safinya, M. Kaplan, S. S. Dana, R. Schaetzing, R. J. Birgeneau, and J. D. Litster, High-resolution x-ray and light-scattering study of critical behavior associated with the nematic-smectic-A transition in 4-cyano4'-octylbiphenyl, Phys. Rev. B 19, 1657 (1979).

[34] G. B. Kasting, C. W. Garland, and K. J. Lushington, Critical heat capacity of octylcyanobiphenyl (8CB) near the nematic-smectic A transition, J. Phys. France 41, 879 (1980).

[35] J. Thoen, H. Marynissen, and W. Van Dael, Temperature dependence of the enthalpy and the heat capacity of the liquid-crystal octylcyanobiphenyl (8CB), Phys. Rev. A 26, 2886 (1982).

[36] A. J. Leadbetter, J. L. A. Durrant, and M. Rugman, The Density Of 4 Octyl-4-Cyano-Biphenyl (8CB), Mol. Cryst. Liq. Cryst. 34, 231 (1977).

[37] G. A. Kausche, E. Pfankuch, and H. Ruska, Die Sichtbarmachung von pflanzlichem Virus im bermikroskop, Naturwissenschaften 27, 292 (1939).

[38] Dowloaded from http://upload.wikimedia.org/wikipedia/commons/4/ 47/TobaccoMosaicVirus.jpg on 31. August 2011.

[39] A. Skoulios, La structure des solutions aqueuses concentres de savon, Advances in Colloid and Interface Science 1, 79 (1967). 
Bibliography

[40] J. F. Nagle, Theory of biomembrane phase transitions, J. Chem. Phys. 58, 252 (1973).

[41] J. F. Nagle, Lipid Bilayer Phase Transition: Density Measurements and Theory, Proceedings of the National Academy of Sciences 70, 3443 (1973).

[42] M. Mihailescu, R. G. Vaswani, E. Jardón-Valadez, F. Castro-Román, J. A. Freites, D. L. Worcester, A. R. Chamberlin, D. J. Tobias, and S. H. White, AcylChain Methyl Distributions of Liquid-Ordered and-Disordered Membranes, Biophys. J. 100, 1455 (2011).

[43] M. Warner and E. M. Terentjev, Nematic elastomers-A new state of matter?, Progress in Polymer Science 21, 853 (1996).

[44] L. Petridis and E. M. Terentjev, Nematic-isotropic transition with quenched disorder, Phys. Rev. E 74, 051707 (2006).

[45] S. M. Clarke, E. M. Terentjev, I. Kundler, and H. Finkelmann, Texture Evolution during the Polydomain-Monodomain Transition in Nematic Elastomers, Macromolecules 31, 4862 (1998).

[46] F. Elias, S. M. Clarke, R. Peck, and E. M. Terentjev, Equilibrium textures in main-chain liquid crystalline polymers, EPL 47, 442 (1999).

[47] P. M. Chaikin and T. C. Lubensky, Principles of condensed matter physics (Cambridge University Press, 1995), first edn.

[48] D. Frenkel and R. Eppenga, Evidence for algebraic orientational order in a two-dimensional hard-core nematic, Phys. Rev. A 31, 1776 (1985).

[49] M. P. Lettinga, E. Barry, and Z. Dogic, Self-diffusion of rod-like viruses in the nematic phase, EPL (Europhysics Letters) 71, 692 (2005).

[50] P. L. Nordio and U. Segre, Magnetic Resonance Spectroscopy. Static Behaviour, in: G. R. Luckhurst and G. W. Gray (Eds.), The Mol. Phys. of Liquid Crystals, 367-384 (Academic Press Inc. (London), 1979).

[51] D. A. Dunmur, Physical Origin of Liquid Crystal Optical Properties, in: S. Elston and R. Sambles (Eds.), The Optics of Thermotropic Liquid Crystals, 5-40 (Taylor and Francis Ltd., 1998).

[52] M. Born and E. Wolf, Principles of Optics (Cambridge University Press, 1999), 7th edn.

[53] P. S. Pershan, Raman studies of orientational order in liquid crystals, in: G. R. Luckhurst and G. W. Gray (Eds.), The Mol. Phys. of Liquid Crystals, 385-410 (Academic Press Inc. (London), 1979). 
[54] W. Maier and A. Saupe, Eine einfache molekulare Theorie des nematischen kristallinflssigen Zustandes, Z. Naturf. A13, 564 (1958).

[55] W. Maier and A. Saupe, Eine einfache molekularstatistische Theorie der nematischen kristallinflssigen Phase. Teil I, Z. Naturf. A14, 882 (1959).

[56] W. Maier and A. Saupe, Eine einfache molekularstatistische Theorie der nematischen kristallinflsigen Phase. Teil II, Z. Naturf. A15, 287 (1960).

[57] R. A. L. Jones, Soft Condensed Matter (Oxford University Press, 2002), first edn.

[58] G. R. Luckhurst and C. Zannoni, Why is the MaierSaupe theory of nematic liquid crystals so successful?, Nature 267, 412 (1977).

[59] A. Saupe, Recent Results in the Field of Liquid Crystals, Angewandte Chemie International Edition in English 7, 97 (1968).

[60] L. Onsager, The effects of shape on the interaction of colloidal particles, Ann. N.Y. Acad. Sci. 51, 627 (1949).

[61] G. J. Vroege and H. N. W. Lekkerkerker, Phase transitions in lyotropic colloidal and polymer liquid crystals, Reports on Progress in Physics 55, 1241 (1992).

[62] N. D. Mermin and H. Wagner, Absence of Ferromagnetism or Antiferromagnetism in One- or Two-Dimensional Isotropic Heisenberg Models, Phys. Rev. Lett. 17, 1133 (1966).

[63] A. C. D. van Enter and S. B. Shlosman, First-Order Transitions for $n$-Vector Models in Two and More Dimensions: Rigorous Proof, Phys. Rev. Lett. 89, 285702 (2002).

[64] P. A. Lebwohl and G. Lasher, Nematic-Liquid-Crystal OrderA Monte Carlo Calculation, Phys. Rev. A 6, 426 (1972).

[65] U. Fabbri and C. Zannoni, A Monte Carlo investigation of the Lebwohl-Lasher lattice model in the vicinity of its orientational phase transition, Mol. Phys. $\mathbf{5 8}, 763$ (1986).

[66] T. Fischer and R. L. C. Vink, The WidomRowlinson mixture on a sphere: elimination of exponential slowing down at first-order phase transitions, Journal of Physics: Condensed Matter 22, 104123 (2010).

[67] F. Wang and D. P. Landau, Efficient, Multiple-Range Random Walk Algorithm to Calculate the Density of States, Phys. Rev. Lett. 86, 2050 (2001). 
Bibliography

[68] F. Wang and D. P. Landau, Determining the density of states for classical statistical models: A random walk algorithm to produce a flat histogram, Phys. Rev. E 64 (2001).

[69] S. M. Shell, P. G. Debenedetti, and A. Z. Panagiotopoulos, An improved Monte Carlo method for direct calculation of the density of states, J. Chem. Phys. 119, 9406 (2003).

[70] N. Metropolis, A. W. Rosenbluth, M. N. Rosenbluth, A. H. Teller, and E. Teller, Equation of State Calculations by Fast Computing Machines, The Journal of Chemical Physics 21, 1087 (1953).

[71] K. Binder, Monte Carlo calculation of the surface tension for two- and threedimensional lattice-gas models, Phys. Rev. A 25, 1699 (1982).

[72] T. Neuhaus and J. S. Hager, 2D Crystal Shapes, Droplet Condensation, and Exponential Slowing Down in Simulations of First-Order Phase Transitions, Journal of Statistical Physics 113, 47 (2003).

[73] L. G. MacDowell, P. Virnau, M. Müller, and K. Binder, The evaporation/condensation transition of liquid droplets, The Journal of Chemical Physics 120, 5293 (2004).

[74] Q. Yan and J. J. de Pablo, Fast Calculation of the Density of States of a Fluid by Monte Carlo Simulations, Phys. Rev. Lett. 90, 035701 (2003).

[75] J.-S. Wang and R. H. Swendsen, Transition Matrix Monte Carlo Method, J. Stat. Phys. 106, 245 (2002).

[76] P. Virnau and M. Muller, Calculation of free energy through successive umbrella sampling, J. Chem. Phys. 120, 10925 (2004).

[77] P. A. Lebwohl and G. Lasher, Nematic-Liquid-Crystal Order-A Monte Carlo Calculation, Phys. Rev. A 7, 2222 (1973).

[78] C. M. Care and D. J. Cleaver, Computer simulation of liquid crystals, Reports on Progress in Physics 68, 2665 (2005).

[79] M. E. J. Newman and G. T. Barkema, Monte Carlo Methods in Statistical Physics (Clarendon Press, Oxford, 1999).

[80] N. V. Priezjev and R. A. Pelcovits, Cluster Monte Carlo simulations of the nematic-isotropic transition, Phys. Rev. E 63, 062702 (2001).

[81] F. Wu, The Potts model, Rev. Mod. Phys. 54, 235 (1982). 
[82] E. Domany, M. Schick, and R. H. Swendsen, First-Order Transition in an xy Model with Nearest-Neighbor Interactions, Phys. Rev. Lett. 52, 1535 (1984).

[83] S. Sinha and S. K. Roy, Finite size scaling and first-order phase transition in a modified XY model, Phys. Rev. E 81, 022102 (2010).

[84] A. Jonsson, P. Minnhagen, and M. Nylén, New critical point for two dimensional XY-type models, Phys. Rev. Lett. 70, 1327 (1993).

[85] H. W. J. Blöte, W. Guo, and H. J. Hilhorst, Phase Transition in a TwoDimensional Heisenberg Model, Phys. Rev. Lett. 88, 047203 (2002).

[86] A. C. D. v. Enter and S. B. Shlosman, Provable First-Order Transitions for Nonlinear Vector and Gauge Models with Continuous Symmetries, Communications in Mathematical Physics 255, 21 (2005).

[87] A. C. D. van Enter, S. Romano, and V. A. Zagrebnov, First-order transitions for some generalized XY models, J. Phys. A 39 (2006).

[88] K. Binder, Theory of first-order phase transitions, Reports on Progress in Physics 50, 783 (1987).

[89] N. Goldenfeld, Lectures on Phase Transitions and the Renormalization Group, Frontiers in Physics (Westview Press, 1992).

[90] V. Privman and M. E. Fisher, Finite-size rounding of first-order transitions, J. Appl. Phys. 57, 3327 (1985).

[91] M. E. Fisher and A. N. Berker, Scaling for first-order phase transitions in thermodynamic and finite systems, Phys. Rev. B 26, 2507 (1982).

[92] K. Binder, Finite size scaling analysis of ising model block distribution functions, Z. Phys. B 43, 119 (1981).

[93] G. Jona-Lasinio, The renormalization group: A probabilistic view, Il Nuovo Cimento B (1971-1996) 26, 99 (1975).

[94] Y. Imry, Finite-size rounding of a first-order phase transition, Phys. Rev. B 21, 2042 (1980).

[95] A. Billoire, R. Lacaze, and A. Morel, A numerical study of finite-size scaling for first-order phase transitions, Nucl. Phys. B 370, 773 (1992).

[96] M. S. S. Challa, D. P. Landau, and K. Binder, Finite-size effects at temperature-driven first-order transitions, Phys. Rev. B 34, 1841 (1986).

[97] R. Potts, Proc. Camb. Phil. Soc. 48, 106 (1952). 
Bibliography

[98] C. Borgs, R. Kotecký, and S. Miracle-Solé, Finite-size scaling for Potts models, J. Stat. Phys. 62, 529 (1991).

[99] Z. Zhang, O. G. Mouritsen, and M. J. Zuckermann, Weak first-order orientational transition in the Lebwohl-Lasher model for liquid crystals, Phys. Rev. Lett. 69, 2803 (1992).

[100] C. Borgs and R. Kotecký, A rigorous theory of finite-size scaling at first-order phase transitions, J. Stat. Phys. 61, 79 (1990).

[101] C. Borgs and R. Kotecký, Finite-size effects at asymmetric first-order phase transitions, Phys. Rev. Lett. 68, 1734 (1992).

[102] D. Jayasri, V. S. S. Sastry, and K. P. N. Murthy, Wang-Landau Monte Carlo simulation of isotropic-nematic transition in liquid crystals, Phys. Rev. E 72, 036702 (2005).

[103] J. M. Fish and R. L. C. Vink, Isotropic-to-nematic transition in confined liquid crystals: An essentially nonuniversal phenomenon, Phys. Rev. E 81, 021705 (2010).

[104] J. Lee and J. M. Kosterlitz, New numerical method to study phase transitions, Phys. Rev. Lett. 65, 137 (1990).

[105] J. Lee and J. M. Kosterlitz, Finite-size scaling and Monte Carlo simulations of first-order phase transitions, Phys. Rev. B 43, 3265 (1991).

[106] W. Janke, Accurate first-order transition points from finite-size data without power-law corrections, Phys. Rev. B 47, 14757 (1993).

[107] D. P. Landau, K. K. Mon, and H. B. Schuettler (Eds.), Recent Developments in Monte Carlo Simulations of First-Order Phase Transitions (Springer-Verlag, Berlin, Heidelberg, 1994).

[108] P. Sheng, Phase Transition in Surface-Aligned Nematic Films, Phys. Rev. Lett. 37, 1059 (1976).

[109] H. Yokoyama, Nematic-isotropic transition in bounded thin films, J. Chem. Soc., Faraday Trans. 2 84, 1023 (1988).

[110] M. M. Telo da gama and P. Tarazona, First-order and continuous transitions in confined liquid crystals, Phys. Rev. A 41, 1149 (1990).

[111] Gama, P. Tarazona, M. P. Allen, and R. Evans, The effect of confinement on the isotropic-nematic transition, Mol. Phys. 71, 801 (1990). 
[112] A. Poniewierski and T. J. Sluckin, Theory of the Nematic-Isotropic Transition in a Restricted Geometry, Liq. Cryst. 2, 281 (1987).

[113] P. Sheng, Boundary-layer phase transition in nematic liquid crystals, Phys. Rev. A 26, 1610 (1982).

[114] D. J. Cleaver and M. P. Allen, Computer simulation of liquid crystal films, Mol. Phys. 80, 253 (1993).

[115] M. C. Lagomarsino, M. Dogterom, and M. Dijkstra, Isotropic-nematic transition of long, thin, hard spherocylinders confined in a quasi-two-dimensional planar geometry, J. Chem. Phys. 119, 3535 (2003).

[116] M. Dijkstra, R. van Roij, and R. Evans, Wetting and capillary nematization of a hard-rod fluid: A simulation study, Phys. Rev. E 63, 051703 (2001).

[117] M. M. Pineiro, A. Galindo, and A. O. Parry, Surface ordering and capillary phenomena of confined hard cut-sphere particles, Soft Matter 3, 768 (2007).

[118] V. R. Roij, M. Dijkstra, and R. Evans, Orientational wetting and capillary nematization of hard-rod fluids, EPL 49, 350 (2000).

[119] R. Paredes, A. I. Fariñas Sánchez, and R. Botet, No quasi-long-range order in a two-dimensional liquid crystal, Phys. Rev. E 78, 051706 (2008).

[120] A. I. Fariñas Sánchez, R. Botet, B. Berche, and R. Paredes, On the critical behaviour of two-dimensional liquid crystals, Condens. Matter Phys. 13, 13601 (2010).

[121] J. M. Kosterlitz and D. J. Thouless, Long range order and metastability in two dimensional solids and superfluids. (Application of dislocation theory), J. Phys. C 5 (1972).

[122] N. G. Almarza, C. Martín, and E. Lomba, Phase behavior of the confined Lebwohl-Lasher model, Phys. Rev. E 82, 011140 (2010).

[123] A. C. D. van Enter and S. B. Shlosman, First-Order Transitions for $n$-Vector Models in Two and More Dimensions: Rigorous Proof, Phys. Rev. Lett. 89, 285702 (2002).

[124] R. L. C. Vink, Liquid Crystals in Two Dimensions: First-Order Phase Transitions and Nonuniversal Critical Behavior, Phys. Rev. Lett. 98, 217801 (2007).

[125] H. H. Wensink and R. L. C. Vink, First-order phase transitions in twodimensional off-lattice liquid crystals, J. Phys.: Condens. Matter 19, 466109 (2007). 
Bibliography

[126] Z. Zhang, O. G. Mouritsen, and M. J. Zuckermann, Weak first-order orientational transition in the Lebwohl-Lasher model for liquid crystals, Phys. Rev. Lett. 69, 2803 (1992).

[127] K. Binder, Critical Properties from Monte Carlo Coarse Graining and Renormalization, Phys. Rev. Lett. 47, 693 (1981).

[128] J. M. Kosterlitz, The critical properties of the two-dimensional xy model, J. Phys. C 7, 1046 (1974).

[129] W. Janke, Logarithmic corrections in the two-dimensional XY model, Phys. Rev. B 55, 3580 (1997).

[130] A. O. Parry and R. Evans, Universal fluctuation-induced corrections to the Kelvin equation for capillary condensation, Journal of Physics A: Mathematical and General 25, 275 (1992).

[131] H. Reich and M. Schmidt, Capillary nematization of hard colloidal platelets confined between two parallel hard walls, J. Phys.: Condens. Matter 19, 326103 (2007).

[132] L. Wu, B. Zhou, C. W. Garland, T. Bellini, and D. W. Schaefer, Heatcapacity study of nematic-isotropic and nematic-smectic-A transitions for octylcyanobiphenyl in silica aerogels, Phys. Rev. E 51, 2157 (1995).

[133] A. Maritan, M. Cieplak, and J. R. Banavar, Nematic-Isotropic Transition in Porous Media, in: G. P. Crawford and S. Žumer (Eds.), Liquid Crystals in Complex Geometries, 483 (Taylor \& Francis, 1996).

[134] T. Bellini, N. A. Clark, C. D. Muzny, L. Wu, C. W. Garland, D. W. Schaefer, and B. J. Oliver, Phase behavior of the liquid crystal $8 C B$ in a silica aerogel, Phys. Rev. Lett. 69, 788 (1992).

[135] X.-l. Wu, W. I. Goldburg, M. X. Liu, and J. Z. Xue, Slow Dynamics of Isotropic-Nematic Phase Transition in Silica Gels, Phys. Rev. Lett. 69, 470 (1992).

[136] B. Zhou, G. S. Iannacchione, C. W. Garland, and T. Bellini, Random-field effects on the nematic-smectic-A phase transition due to silica aerosil particles, Phys. Rev. E 55, 2962 (1997).

[137] G. Iannacchione and D. Finotello, Confinement and orientational study at liquid crystal phase transitions, Liq. Cryst. 14, 1135 (1993).

[138] S. Tripathi, C. Rosenblatt, and F. M. Aliev, Orientational susceptibility in porous glass near a bulk nematic-isotropic phase transition, Phys. Rev. Lett. 72, 2725 (1994). 
[139] D. Ge, L. Yang, Y. Li, and J. Zhao, Hydrophobic and thermal insulation properties of silica aerogel/epoxy composite, Journal of Non-Crystalline Solids 355, 2610 (2009).

[140] Y. Imry and S. K. Ma, Random-Field Instability of the Ordered State of Continuous Symmetry, Phys. Rev. Lett. 35, 1399 (1975).

[141] N. A. Clark, T. Bellini, R. M. Malzbender, B. N. Thomas, A. G. Rappaport, C. D. Muzny, D. W. Schaefer, and L. Hrubesh, X-ray scattering study of smectic ordering in a silica aerogel, Phys. Rev. Lett. 71, 3505 (1993).

[142] Y. Imry and M. Wortis, Influence of quenched impurities on first-order phase transitions, Phys. Rev. B 19, 3580 (1979).

[143] A. Aharony and A. B. Harris, Absence of Self-Averaging and Universal Fluctuations in Random Systems near Critical Points, Phys. Rev. Lett. 77, 3700 (1996).

[144] S. Wiseman and E. Domany, Finite-Size Scaling and Lack of Self-Averaging in Critical Disordered Systems, Phys. Rev. Lett. 81, 22 (1998).

[145] S. Wiseman and E. Domany, Self-averaging, distribution of pseudocritical temperatures, and finite size scaling in critical disordered systems, Phys. Rev. E 58, 2938 (1998).

[146] T. Bellini, C. Chiccoli, P. Pasini, and C. Zannoni, Lattice Spin Models of Liquid Crystals in Aerogels, Mol. Cryst. Liq. Cryst. Science and Technology. Section A. Mol. Cryst. Liq. Cryst. 290, 227 (1996).

[147] T. Bellini, M. Buscaglia, C. Chiccoli, F. Mantegazza, P. Pasini, and C. Zannoni, Nematics with Quenched Disorder: What Is Left when Long Range Order Is Disrupted?, Phys. Rev. Lett. 85, 1008 (2000).

[148] D. J. Cleaver, S. Kralj, T. J. Sluckin, and M. P. Allen, The Random Anisotropy Nematic Spin Model, in: G. P. Crawford and S. Žumer (Eds.), Liquid Crystals in Complex Geometries, 467 (Taylor \& Francis, 1996).

[149] J. Chakrabarti, Simulation Evidence of Critical Behavior of Isotropic-Nematic Phase Transition in a Porous Medium, Phys. Rev. Lett. 81, 385 (1998).

[150] A. Maritan, M. Cieplak, T. Bellini, and J. R. Banavar, Nematic-Isotropic Transition in Porous Media, Phys. Rev. Lett. 72, 4113 (1994).

[151] M. Rotunno, M. Buscaglia, C. Chiccoli, F. Mantegazza, P. Pasini, T. Bellini, and C. Zannoni, Nematics with Quenched Disorder: Pinning out the Origin of Memory, Phys. Rev. Lett. 94, 097802 (2005). 
Bibliography

[152] M. Buscaglia, T. Bellini, C. Chiccoli, F. Mantegazza, P. Pasini, M. Rotunno, and C. Zannoni, Memory effects in nematics with quenched disorder, Phys. Rev. E 74, 011706 (2006).

[153] R. Brout, Statistical Mechanical Theory of a Random Ferromagnetic System, Phys. Rev. 115, 824 (1959).

[154] D. E. Feldman, Quasi-Long-Range Order in Nematics Confined in Random Porous Media, Phys. Rev. Lett. 84, 4886 (2000).

[155] T. Nattermann, Theory of the Random Field Ising Model, in: A. P. Young (Ed.), Spin Glasses and Random Fields, 277 (World Scientific, Singapore, 1998).

[156] B. Khasanov, Isotropic phase of nematics in porous media, JETP Lett. 81, 24 (2005).

[157] C. Monthus and T. Garel, Distribution of pseudo-critical temperatures and lack of self-averaging in disordered Poland-Scheraga models with different loop exponents, The European Physical Journal B - Condensed Matter and Complex Systems 48, 393 (2005).

[158] D. S. Fisher, Scaling and critical slowing down in random-field Ising systems, Phys. Rev. Lett. 56, 416 (1986).

[159] Villain, J., Commensurate-incommensurate transition with frozen impurities, J. Physique Lett. 43, 551 (1982).

[160] K. Eichhorn and K. Binder, Finite-Size Scaling Study of the Three-State Potts Model in Random Fields: Evidence for a Second-Order Transition, Europhys. Lett. 30, 331 (1995). 


\section{Lebenslauf}

Persönliche Daten

Name: Jonathan Fish

Geburtstag und -ort: 23.05.1986 in London, Vereinigtes Königreich

Adresse:

Albert Einstein Str. 40, 37075 Göttingen

Nationalität:

Britisch

Ausbildung

$1997-2004$

Studium

$2004-2008$

$2006-2007$

Juli 2008

Juli 2008
Manchester Grammar School, Manchester

Studium Physik, University of Bristol

Studium Physik, Technische Universität Darmstadt

Master of Physics, Note: upper second

Beginn Promotion Physik bei Dr. Richard Vink

Phase Behavior of Liquid Crystals in Confinement

am Institut für Theoretische Physik, Georg-August Universität Göttingen;

\section{Masterarbeit}

Jonathan M. Fish, Low Voltage Phosphors, Masterarbeit (2008).

\section{Publikationen}

1. J. M. Fish and R. L. C. Vink, Finite-size effects at first-order isotropic-tonematic transitions, Phys. Rev. B 80, 014107 (2009).

2. J. M. Fish and R. L. C. Vink, Isotropic-to-nematic transition in confined liquid crystals: An essentially nonuniversal phenomenon, Phys. Rev. E 81, 021705 (2010).

3. J. M. Fish and R. L. C. Vink, Nematics with quenched disorder: violation of self-averaging, Phys. Rev. Lett. 105, 147801 (2010). 



\section{Curriculum Vitae}

\section{Personal Details}

Name: Jonathan Fish

Date of birth: 23.05.1986, London, United Kingdom

Address: $\quad$ Albert Einstein Str. 40, 37075 Göttingen

Nationality: British

\section{Education}

1997 - 2004 Manchester Grammar School

\section{University Studies}

2004 - 2008 Study of physics, University of Bristol

2006 - 2007 Study of physics, Technische Universität Darmstadt

July 2008 Master of Physics, Grade: upper second

July 2008 Begin PhD in Physics under Dr. Richard Vink

Liquid Crystals with Quenched Disorder

at the Institut für Theoretische Physik, Georg-August Universität Göttingen;

\section{Masters Subject}

Jonathan M. Fish, Low Voltage Phosphors, Masters thesis (2008).

\section{Publications}

1. J. M. Fish and R. L. C. Vink, Finite-size effects at first-order isotropic-tonematic transitions, Phys. Rev. B 80, 014107 (2009).

2. J. M. Fish and R. L. C. Vink, Isotropic-to-nematic transition in confined liquid crystals: An essentially nonuniversal phenomenon, Phys. Rev. E 81, 021705 (2010).

3. J. M. Fish and R. L. C. Vink, Nematics with quenched disorder: violation of self-averaging, Phys. Rev. Lett. 105, 147801 (2010). 
